OPEN ACCESS

Edited by:

Giovanni Mirabella,

Sapienza Università di Roma, Italy

Reviewed by:

Wei Peng Teo,

Deakin University, Australia

Pietro Cipresso,

Istituto Auxologico Italiano (IRCCS),

Elisa Pedroli,

Istituto Auxologico Italiano (IRCCS),

Italy

Giovanni Albani,

Istituto Auxologico Italiano (IRCCS),

Italy

${ }^{*}$ Correspondence:

Filippo Cavallo

filippo.cavallo@santannapisa.it

Specialty section

This article was submitted to

Neural Technology,

a section of the journal

Frontiers in Neuroscience

Received: 20 June 2017

Accepted: 21 September 2017

Published: 06 October 2017

Citation:

Rovini E, Maremmani $C$ and Cavallo $F$

(2017) How Wearable Sensors Can

Support Parkinson's Disease

Diagnosis and Treatment: $A$

Systematic Review.

Front. Neurosci. 11:555.

doi: 10.3389/fnins.2017.00555

\section{How Wearable Sensors Can Support Parkinson's Disease Diagnosis and Treatment: A Systematic Review}

\author{
Erika Rovini ${ }^{1}$, Carlo Maremmani ${ }^{2}$ and Filippo Cavallo ${ }^{1 *}$ \\ ${ }^{1}$ The BioRobotics Institute, Scuola Superiore Sant'Anna, Pontedera, Italy, ${ }^{2}$ U.O. Neurologia, Ospedale delle Apuane (AUSL \\ Toscana Nord Ovest), Massa, Italy
}

Background: Parkinson's disease (PD) is a common and disabling pathology that is characterized by both motor and non-motor symptoms and affects millions of people worldwide. The disease significantly affects quality of life of those affected. Many works in literature discuss the effects of the disease. The most promising trends involve sensor devices, which are low cost, low power, unobtrusive, and accurate in the measurements, for monitoring and managing the pathology. Objectives: This review focuses on wearable devices for PD applications and identifies five main fields: early diagnosis, tremor, body motion analysis, motor fluctuations (ON-OFF phases), and home and long-term monitoring. The concept is to obtain an overview of the pathology at each stage of development, from the beginning of the disease to consider early symptoms, during disease progression with analysis of the most common disorders, and including management of the most complicated situations (i.e., motor fluctuations and long-term remote monitoring). Data sources: The research was conducted within three databases: IEEE Xplore ${ }^{\circledR}$, Science Direct ${ }^{\circledR}$, and PubMed Central ${ }^{\circledR}$, between January 2006 and December 2016. Study eligibility criteria: Since 1,429 articles were found, accurate definition of the exclusion criteria and selection strategy allowed identification of the most relevant papers. Results: Finally, 136 papers were fully evaluated and included in this review, allowing a wide overview of wearable devices for the management of Parkinson's disease.

Keywords: Parkinson's disease, wearable sensors, motion analysis, early diagnosis, tremor, motor fluctuations, monitoring, telemedicine

\section{INTRODUCTION}

Parkinson's disease (PD) is a complex neurodegenerative disorder that has a usually asymmetric onset, characterized by typical motor symptoms as bradykinesia, hypo-/akinesia, muscular rigidity, and resting tremor (Fahn, 2008). Although the pathology is generally diagnosed on the basis of these motor symptoms, many non-motor manifestations (NMMs) are commonly evident and they may sometimes be more disabling of motor disturbances, such as olfactory disturbances, autonomic dysfunction, sleep fragmentation, depression, and dementia (Wolters, 2008). Some NMMs (e.g., sleep disorders, bladder disturbances, gastrointestinal symptoms, olfactory symptoms) may occur throughout the entire course of the disease, even if cognitive symptoms such as hallucinations and dementia tend to occur late in the PD. The disease is difficult to detect and treat promptly, as it shows a wide variability in the clinical expression (Fahn, 2008) as well as in the somatic 
symptom progression (Dickson and Grünewald, 2004; Caslake et al., 2013; de Lau et al., 2014; Szewczyk-Krolikowski et al., 2014). Over the past three decades, the knowledge of PD has increased significantly, with particular interest on the premotor phase and novel therapeutic and diagnostic approaches (Korczyn and Gurevich, 2010). Currently, experts recognize the need to redefine the research criteria for the diagnosis of this complex disease by considering clinical features, pathological findings, and genetics or molecular mechanisms (Mirelman et al., 2011; Berg et al., 2013). Recent studies demonstrate that several NNMs (e.g., rapid eye movement sleep behavior disorders, hyposmia, constipation, depression) are correlated to the neuropathological changes in the brain and they can anticipate the motor manifestations of the disease by $5-7$ years. Furthermore, the study of the pre-motor phase could lead the research for predictive biomarkers and risk or protective factors for PD (Tolosa and Pont-Sunyer, 2011; Palma and Kaufmann, 2014). Today, PD diagnosis is based on the assessment of motor (and non-motor) symptoms, typically during neurological visual examinations, but the diagnostic methods and disease progression monitoring approaches remain suboptimal for PD management (Kassubek, 2014). This is particularly true when cofactors such as greater age, poor cognition, and worse mobility are manifested (Hu et al., 2011). During the test for PD diagnosis, in fact, the neurologist watches the patient perform specific tasks and assigns scores for each of them as required and defined in the Unified Parkinson's Disease Rating Scale (UPDRS) (Fahn and Elton, 1987) or its updated version, the Movement Disorder Society-sponsored revision of the UPDRS (MDS-UPDRS) (Goetz et al., 2008). The Hoehn and Yahr scale (HY) (Hoehn and Yahr, 1967) instead includes stages 1-5, and it is used to assign an overall score to the patient on the basis of the pathological progress. All these clinical scales are subjective; this fact leads to high inter-rater variability among different neurologists or different medical centers, as well as high intra-rater variability over time. The correct diagnosis of $\mathrm{PD}$ is of vital importance for adequate prognosis and treatment, although a study reveals that $\sim 25 \%$ of diagnoses are incorrect, particularly when essential tremor, vascular Parkinsonism, and atypical Parkinsonian syndromes are manifested (Tolosa et al., 2006). An exhaustive study of the pathology, including a more accurate knowledge of its clinical appearance and other tests such as olfactory exam and magnetic resonance imaging (MRI), could guide the correct diagnosis (Tolosa et al., 2006). The treatment for PD is still a matter of debate, especially in the early phases. Common sense says that the therapy must be personalized and adapted to the individual needs of PD patients to provide the best medical care and treat the predominant symptoms (Ossig and Reichmann, 2015). Early and accurate diagnosis of PD may improve the long-term quality of life (QoL) for PwPD, while misdiagnosing a patient causes delay in receiving the appropriate treatment plan.

In this context, the use of smart technologies for PD applications has increased in recent years. In particular, wearable sensors are fundamental in helping clinicians perform early diagnosis, differential diagnosis, and objective quantification of symptoms over time. A growing number of papers concerning this topic during the last decade also demonstrate the increasing development and use of such wearable technologies. For example, the use of inertial sensors such as accelerometers (ACC) and gyroscopes (GYRO), combined with advances in short-range communication technologies (i.e., Bluetooth, Zigbee), is now feasible and meets the needs of people with chronic disorders by featuring low power consumption, unobtrusiveness, light weight, and ease of use (Bonato, 2010). Wearable sensors have demonstrated their potential power for PD diagnosis (Butt et al., 2017) and management (Rovini et al., 2016), as well as for other pathologies (e.g., post-stroke, neck injuries) (Rodgers et al., 2015) or to monitor pharmacological trials (Henderson et al., 2016). In terms of pharmacological treatment, levodopa (Ldopa) is currently the most used and effective medication for PD, even if several side effects result from it, especially motor fluctuations and dyskinesias (Chou, 2008). When Ldopa-related side effects are difficult to control, surgical therapies such as neuromodulation using deep brain stimulation (DBS) (Rissanen et al., 2015) can be applied, while at the same time, other potential solutions (e.g., biological therapies) are emerging (Strauss et al., 2014). To redefine new metrics for early diagnosis, differential diagnosis, and quantification of symptoms, the development of a system for objective assessment of the pathology to identify motor dysfunctions, which are imperceptible upon expert clinical exam, is required (Scanlon et al., 2013). Finally, it is important to consider also the social aspects that are involved with a disabling pathology such as PD. The burden of care among caregivers of $\mathrm{PwPD}$ considerably increases with age and disease progression and is linked to the period and level of assistance required (Razali et al., 2011), whereas non-motor symptoms, especially cognitive decline, play a prevalent role in caregivers' grief (Carter et al., 2012). To reduce the burden of caretakers, a recent study (Megalingam et al., 2014) proposes a wearable health monitoring system that can measure heart rate, temperature, electrocardiogram (ECG), tilt, and fall of the homebound patients and can send a notification via smartphone to the caregivers if a critical situation is occurring. Such a system would enable remote assistance.

The aim of this review is to provide readers with broad scientific and technological information about the use and challenges of wearable sensor technologies for PD applications. This paper details the investigation of the typology of wearable sensors, fields of application, processing approaches, and experimental methodologies. Such a complete overview of PD wearable technology makes this paper highly suitable for scientists with both clinical and technical background. In particular, this paper provides a review of the typologies of wearable sensors that were investigated and adopted for PD applications in the last decade, and it describes implemented experimental protocols, the subjects of the studies, extracted features, and performance of classifiers. Such wearable technologies are organized with respect to five critical fields of application that cover the entire pathology progression: (1) early diagnosis, (2) tremor, (3) body motion analysis, (4) motor fluctuations and ON-OFF phases, and (5) home and long-term monitoring. For each topic, the existing systems, found using the methodology described in the next paragraph, were investigated. 
The results are presented here, and recommendations for further development and discussion of future trends are provided as well.

\section{METHODS}

\section{Search Strategy}

An electronic database search was performed from September 2016 to December 2016 using IEEE Xplore ${ }^{\circledR}$, Science Direct ${ }^{\circledR}$, and PubMed Central ${ }^{\circledR}$ to identify articles concerning the use of wearable sensors for PD applications. Specifically, the terms and key words used for the literature research were ("Parkinson") AND ("wearable" OR "inertial" OR "accelerometer" OR "acceleration" OR "gyroscope" OR "EMG” OR "EEG” OR "ECG” OR "GSR" OR "clothes") located within title and/or abstract. Only original, full-text articles published in English, between January 2006 and December 2016, which discussed the use of wearable sensors for PD applications, were included in the review. Obtained in the research were 485 references from IEEE Xplore ${ }^{\circledR}, 653$ references from Science Direct ${ }^{\circledR}$, and 291 references from PubMed Central ${ }^{\circledR}$. Five major applications were identified: early diagnosis, tremor detection, analysis of the motor performances, analysis of motor fluctuations (on/off phases), and home and long-term monitoring. Papers were screened from three independent reviewers (i.e., the authors) and disagreements were solved through meetings and discussions. Finally, the selected papers were classified on the basis of the application area. Data abstracted from the papers and reported in Tables 1-9 considered: the used technological solutions and typology of sensors, their placement over the body and the sampling frequency; the experimental protocol adopted; the subjects involved, according to their pathology and their health status; the performed analysis, including the extracted features, the applied statistical methods, the implemented classifiers and the main findings for each work. Particular attention was focused on the classifiers performance because they can synthetically represent the robustness of the technology proposed for a specific PD application.

\section{Selection Criteria}

First, duplicated references were excluded. Then, during the screening procedure, items were excluded if they (i) were an abstract, a short communication, a review article, or a chapter from a book; (ii) were not written in the English language; (iii) were from years prior to 2010 only for sensors other than inertial (i.e., EMG, EEG, ECG, GSR) because they did not concern wearable devices. Eight hundred and forty-seven references were fully assessed during the evaluation procedure, and papers were excluded if (1) they did not use any type of wearable sensors; (2) they did not appear appropriate for this review after the reading of title and abstract; (3) they did not involve patients with Parkinson's Disease; (4) they were not full access; and (5) they involved a number of PD patients $<10$, due to the low level of reliability and statistical validity that can be obtained from their results. In addition, if multiple papers written by the same author had similar content, papers published in journals were selected instead of papers presented at conferences. Furthermore, if multiple papers written by the same author with similar content were presented at conferences, the most recent paper was selected. Finally, 136 papers were fully evaluated and included in this review (Figure 1).

\section{RESULTS}

Of the 136 fully evaluated papers, $33(24.3 \%)$ were published in 2016, and $73(53.7 \%)$ were published during the last 3 years. This result confirmed the increasing interest for wearable sensors in PD applications (Figure 2A). Eleven papers were appropriate for further applications, which resulted in a total of 147 papers. Among the applications covered by this review, the majority of the papers (61.2\%) focused on body motion analysis (Figure 2B) and in particular on gait analysis, which resulted in the most investigated task (37 papers, 25.2\%) (Figure 2C). Finally, regarding the number of PD patients involved in the studies, even if works that recruited less than 10 PwPD were excluded from this review, the majority of the research efforts (47.1\%) included fewer than 15 PwPD (Figure 2D). Thus, clinical validation of the proposed solutions is still a matter of debate. Because of the significant number of papers included in the review process, only papers published in journals (58.8\%) will be shown in detail in the tables presented here, whereas the full dataset of articles, including 56 papers from conferences, will be uploaded as Supplementary Data online.

\section{Application 1: Early Diagnosis}

Only 5 papers that deal with early diagnosis were obtained by following the research criteria of this review (Table 1). Posture detection systems were investigated because symptoms of postural instability are frequent in the early stage of PD and can lead to complications from festination in the next phases of the pathology. Postural sway performance seems to be a biological marker for prodromal PD (Chen et al., 2014), as it results in an abnormal quite stance in subjects with untreated PD (Mancini et al., 2011).

In contrast, Brodie et al. (2014) proposed to analyse new features extracted from gait, which could represent a biomarker for PD. Jerk, harmonic stability, and oscillation range measured by accelerometers on the pelvis and head were significant measures to distinguish early PwPD when compared to similar measurements for age-matched healthy control (HC) subjects. Sant'Anna et al. (2011) also analyzed gait, focusing on both leg movements and arm swing. They demonstrated that asymmetry between left and right sides in PwPD is higher than in $\mathrm{HC}$, particularly for upper limbs. Different indexes for asymmetry assessment proposed in literature were compared, since this characteristic results in one of the first motor symptoms of the disease. Perumal and Sankar (2016) also considered selected features extracted by gait analysis as possible biomarkers for early diagnosis of PD, because the features enabled good discrimination between PwPD and HC. Perumal and Sankar also analyzed tremor in the frequency domain to differentiate between Parkinsonian tremor and atypical Parkinsonism. They found that different typos of tremor occurred in different frequency bands; particularly, resting tremor occurred during the early stages of 
Rovini et al.

Wearable Sensors for Parkinson's Disease

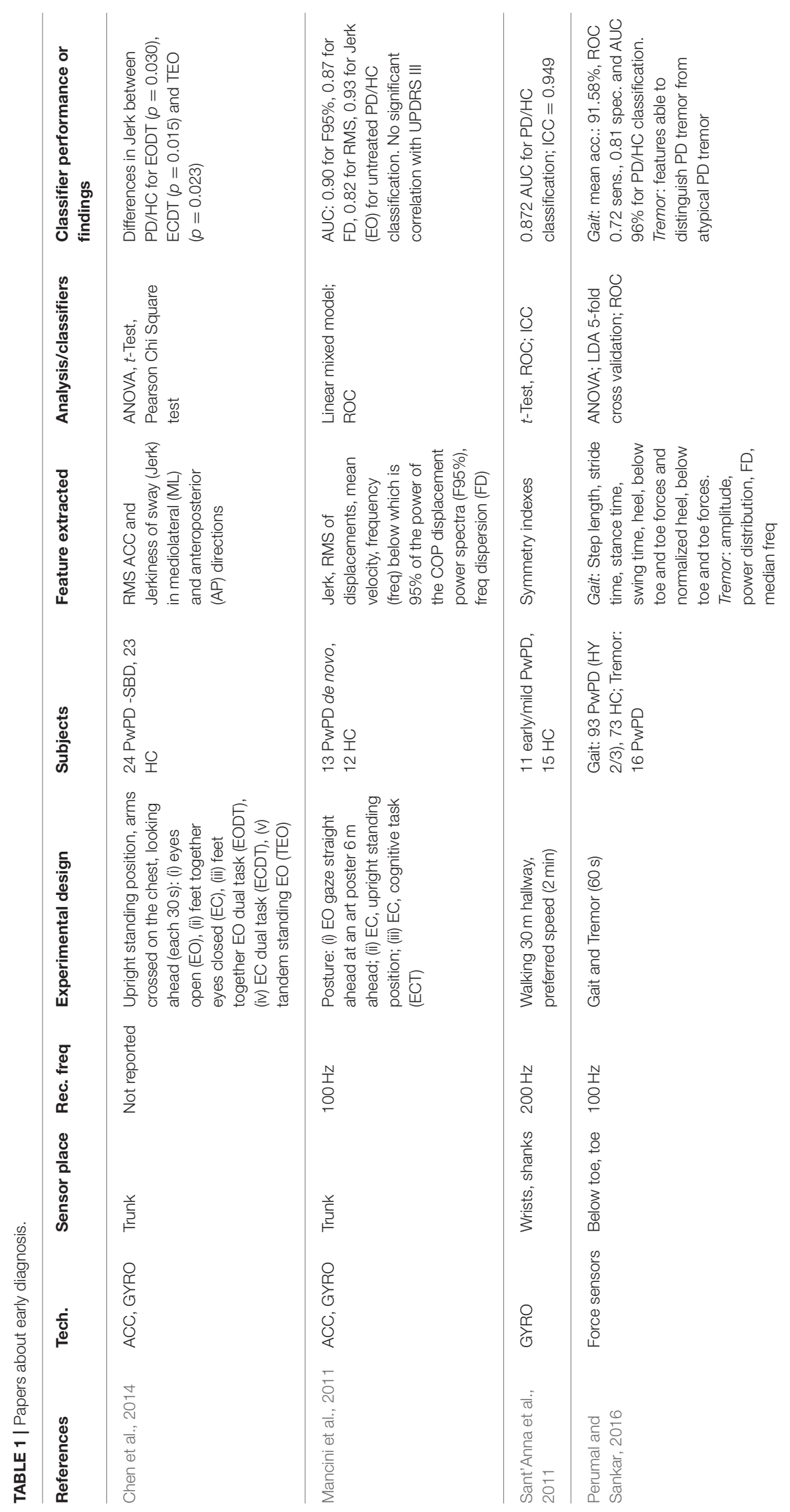

Frontiers in Neuroscience | www.frontiersin.org

4

October 2017 | Volume 11 | Article 555 


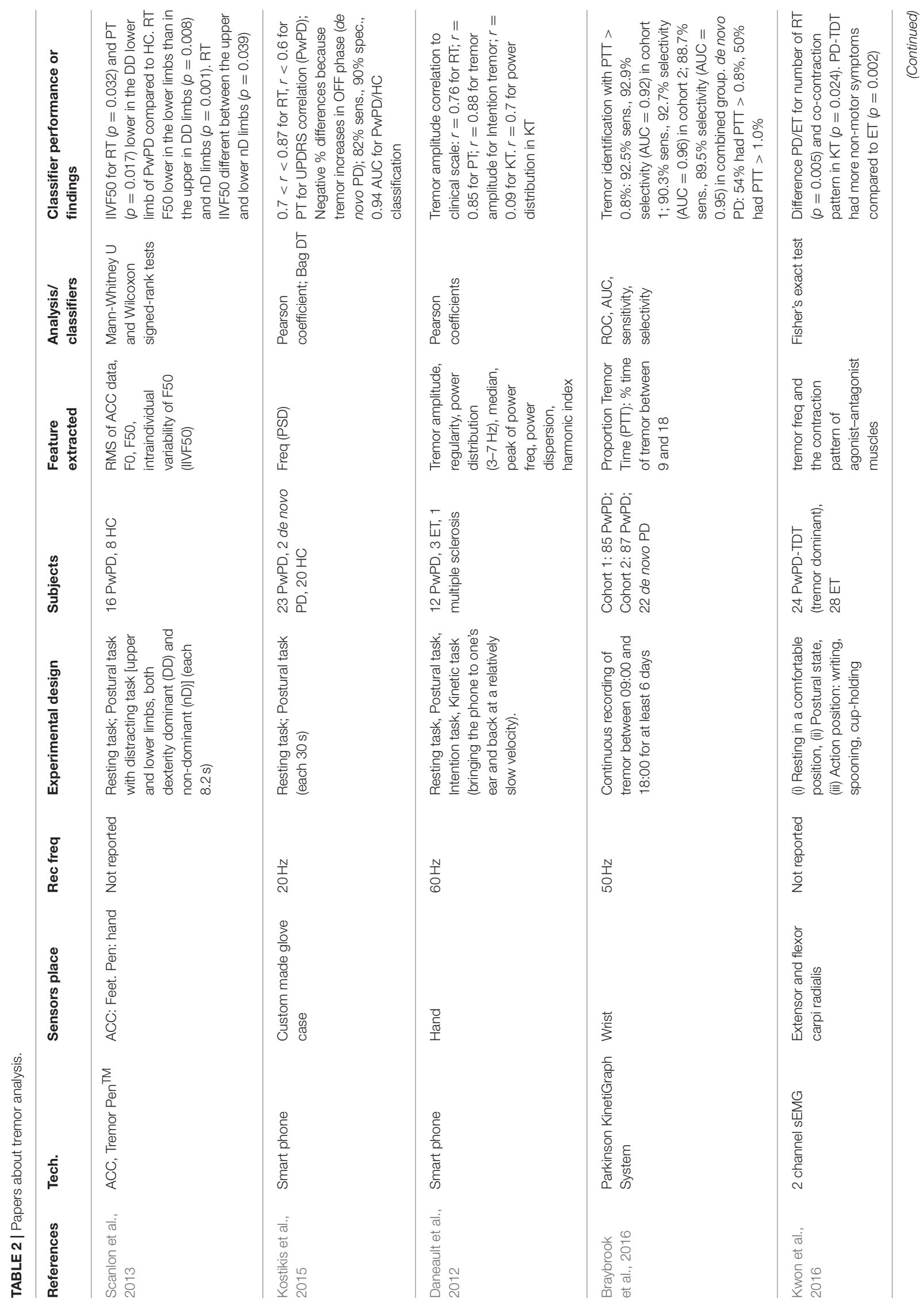




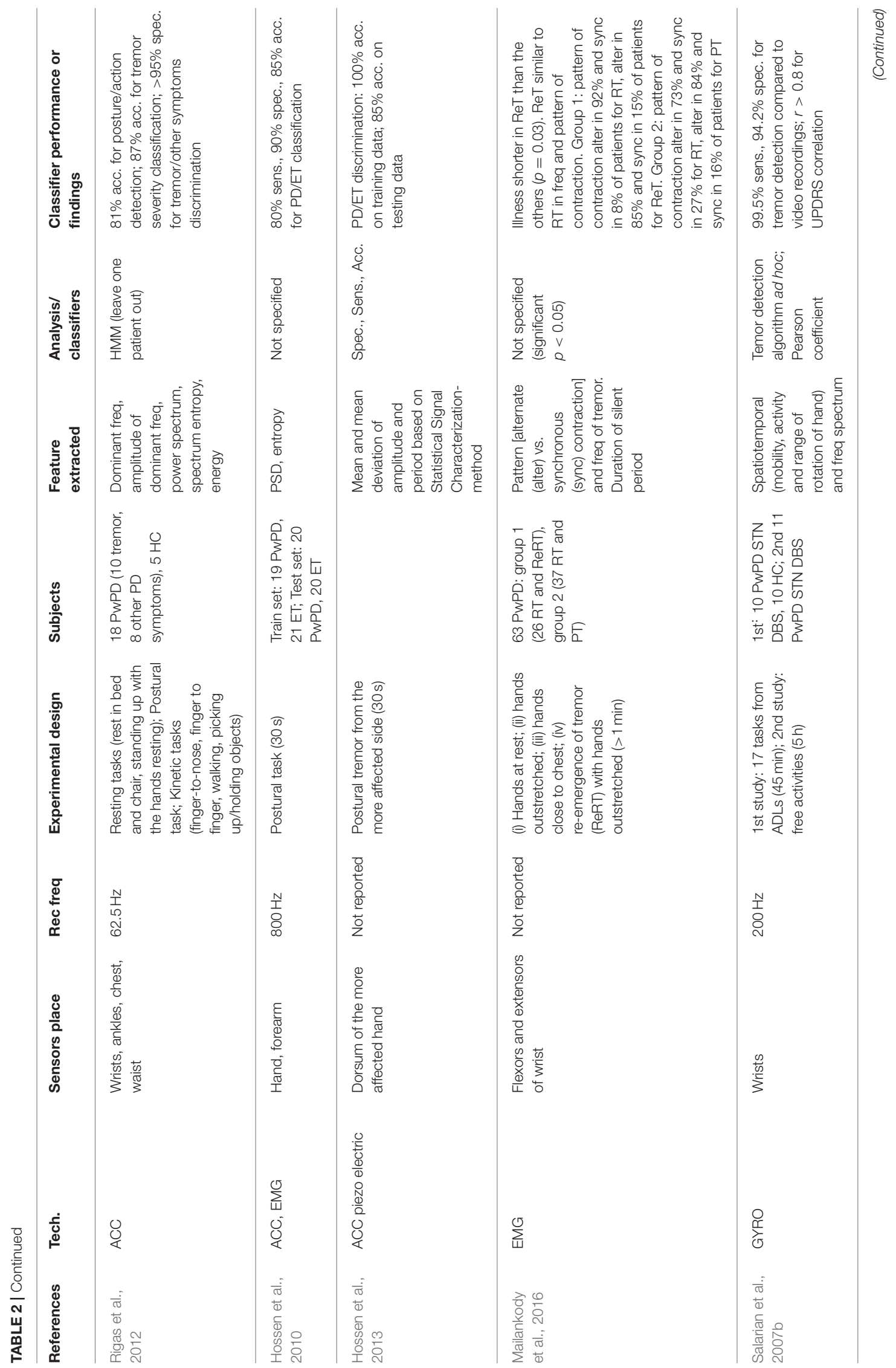




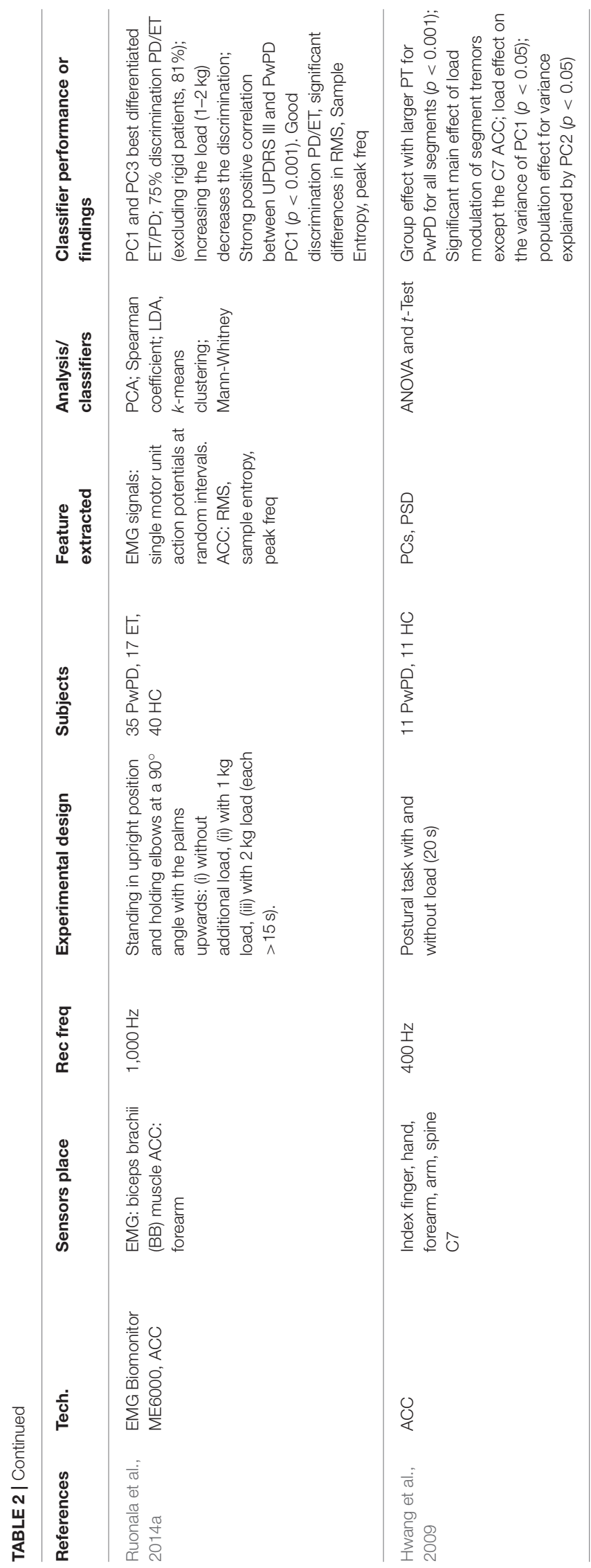

the disease, appearing at the onset in approximately $70 \%$ of patients.

\section{Recommendations and Trends}

The research for technological solutions able to address the early identification of PD is lacking, as demonstrated by the small number of papers found according to the inclusion criteria of this review. The works specifically focused on posture, gait, and asymmetry analyses. The idea is to recognize pathologically abnormal postures as soon as possible, from the prodromal phase of the pathology (Chen et al., 2014), to allow intervention at the earliest stage of PD. To obtain accurate results, the studies must be validated on a large dataset, involving a wide number of subjects with PD at only a mild idiopathic clinical stage of the disease (Brodie et al., 2014). The study of Perumal and Sankar (2016), for example, aimed to accomplish early diagnosis, but it involved PwPD with HY stage 2-3, which is an inappropriate dataset of patients. To investigate the early stage of the disease, only subjects with minimal motor abnormalities must be included in the studies (Sant'Anna et al., 2011), even if recruitment of such patients is difficult because they often do not go to the doctor until symptoms are already widely manifested. In this sense, large prevention and screening programs to identify patients at risk to develop the disease should be recommended and investigated, promoting the early diagnosis of pathology with positive and effective consequences on the therapeutic treatments.

\section{Application 2: Tremor}

Tremor is the most common symptom of PD, resulting in 26 papers in this review; it appears in 70\% of patients and typically involves one side only, at the beginning. Resting tremor (RT) is the prevalent type, and it appears generally when limbs are not intentionally moved, when patient is sitting (typically pillrolling tremor of the hands) or walks with arms dangling. Other typos of tremor are postural tremor (PT) that occurs when the body part is contracted against gravity, kinetic or action tremor (KT) that is task-specific, limited to duration of performing a particular task (e.g., writing), essential tremor (ET) which can overlap the frequency band of RT but is associated with a movement disorder different from PD, and physiological tremor which is present in healthy subjects. Tremor is a complex cerebello-thalamo-cortical phenomenon, but the specific role of the cerebellum in suppressing or generating tremor remains unclear. As the assessment of tremor is currently based on the visual examination of a neurologist, technological solutions able to quantify the gravity of the disease, and efficacy of the therapy appear to provide an optimal solution that offers low invasiveness and high reliability (Scanlon et al., 2013). Correct diagnosis of different tremors is important because the treatment depends on the specific etiology of each tremor type. However, currently reported misdiagnosis between RT and ET may occur in $20-30 \%$ of the cases (Ghassemi et al., 2016; Surangsrirat et al., 2016). In literature, several works have analyzed the use of an inertial measurement unit (IMU) and other sensors, such as electromyography (EMG), which can be complementary in detecting tremor. These sensors can be attached to different parts 


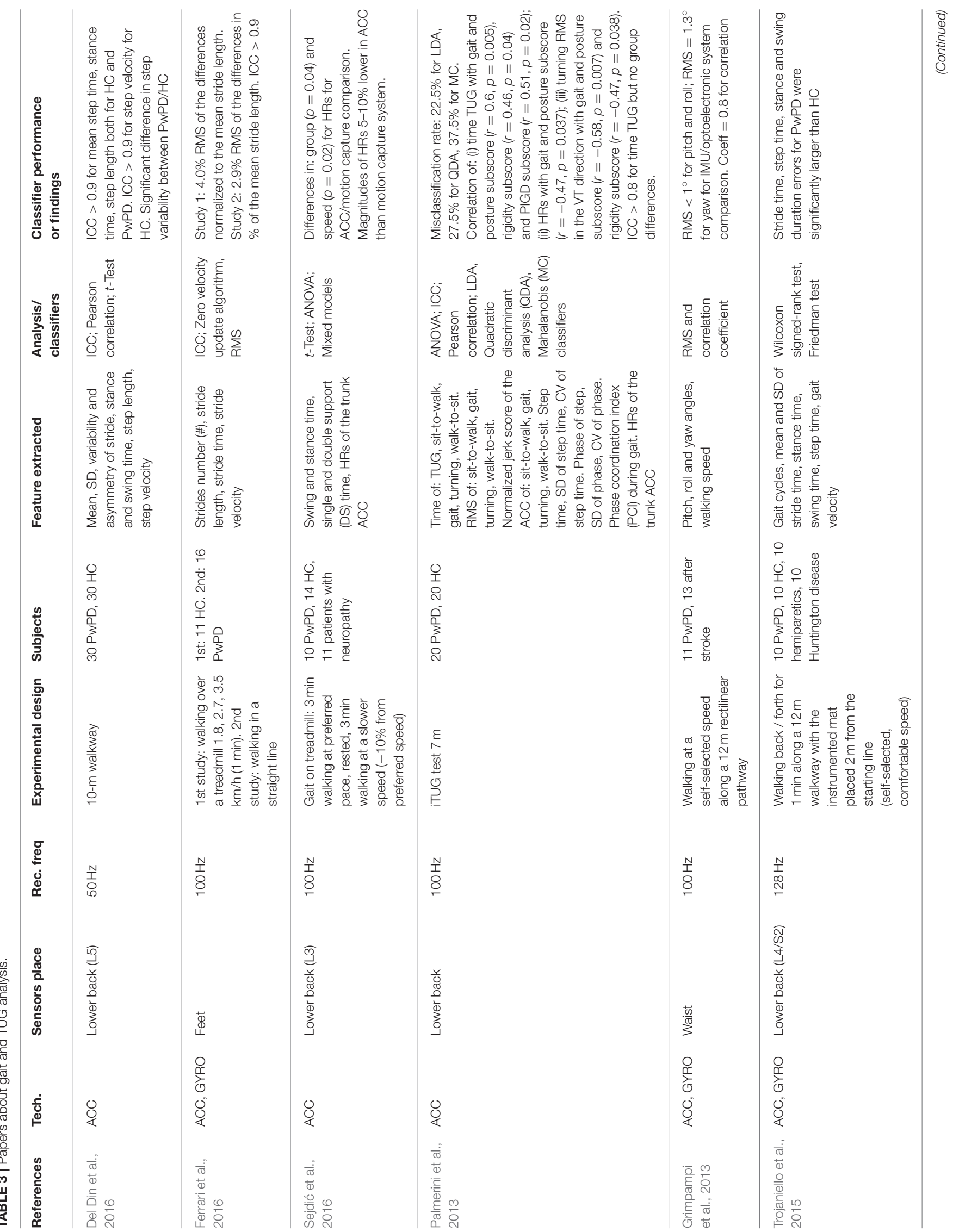




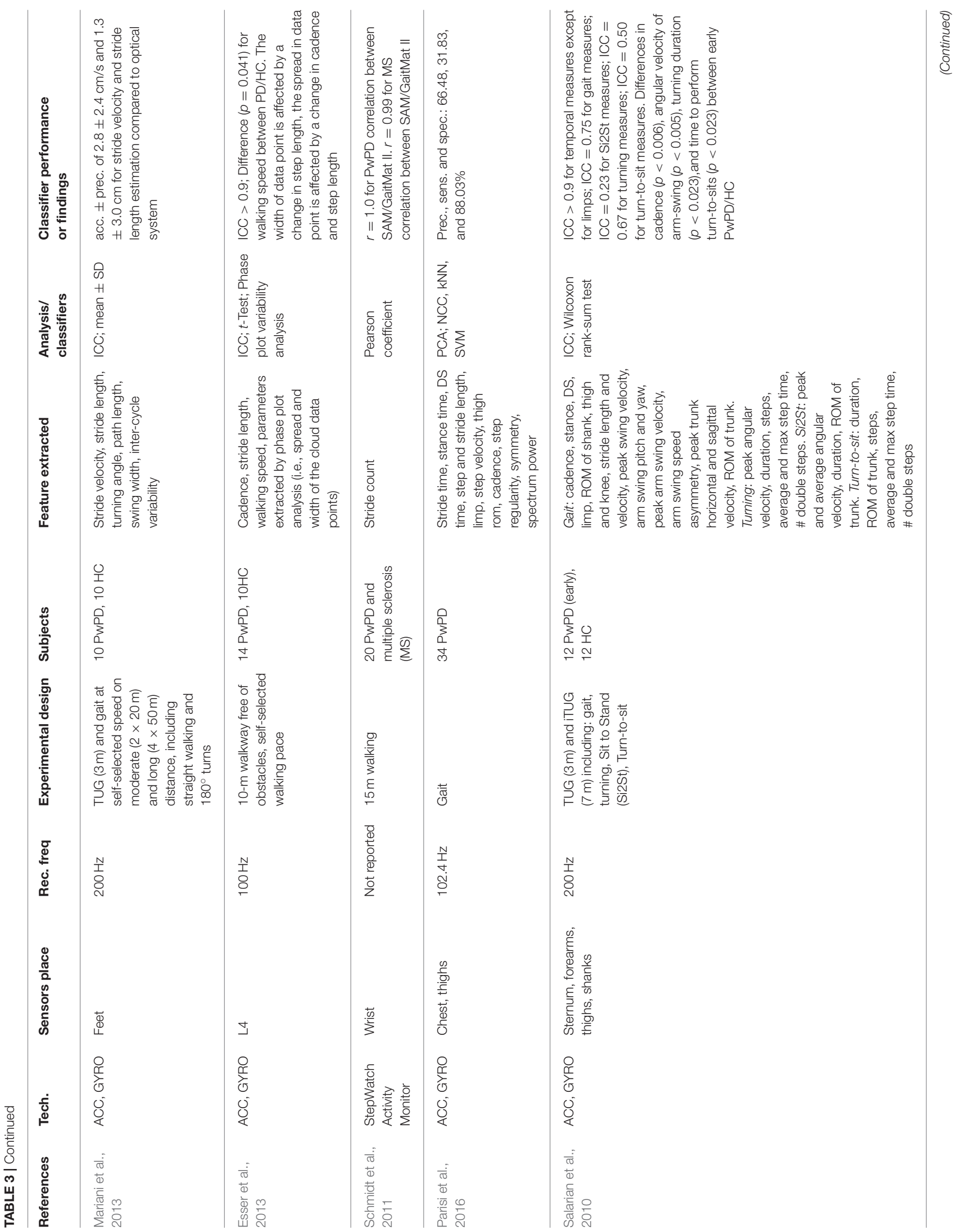




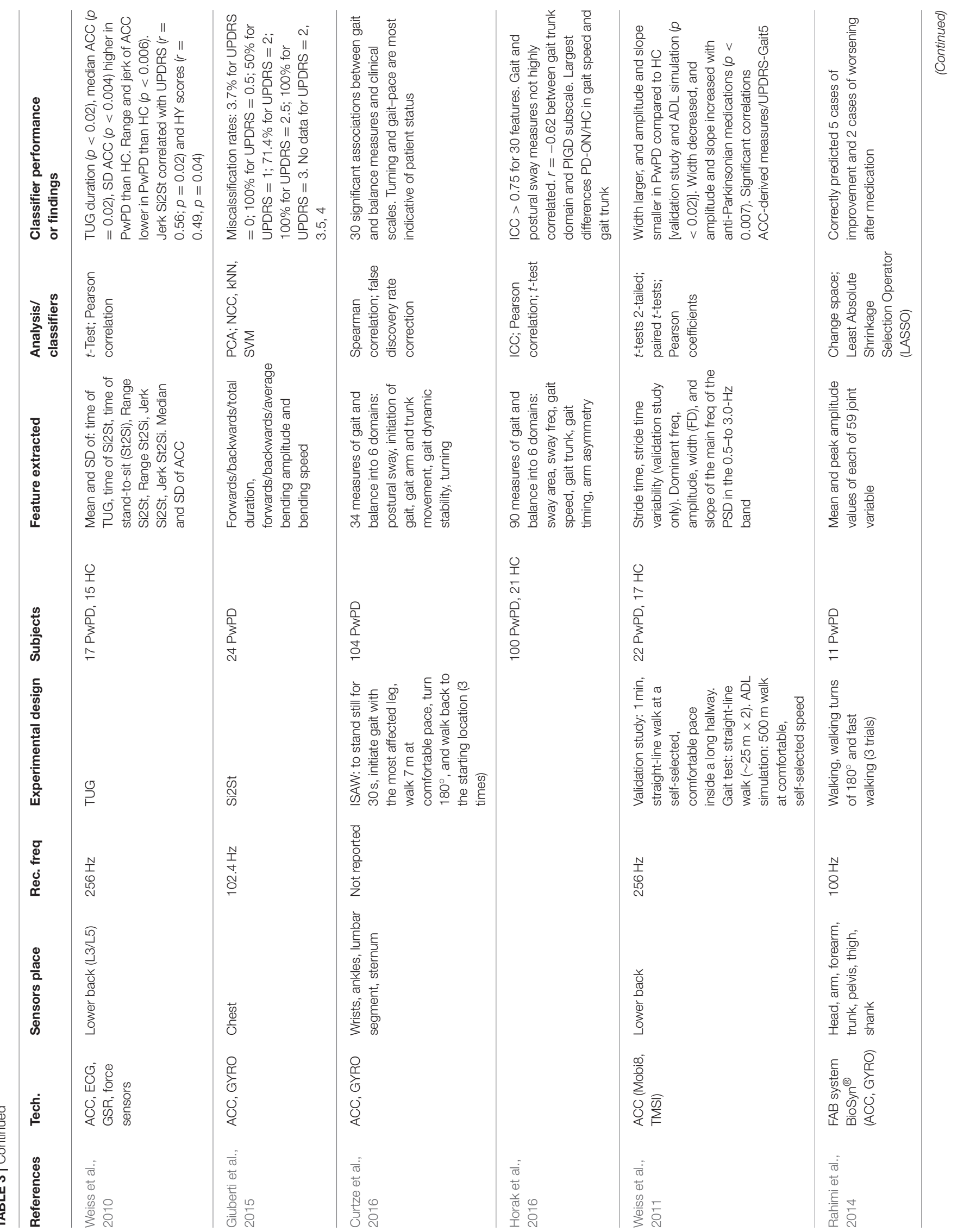




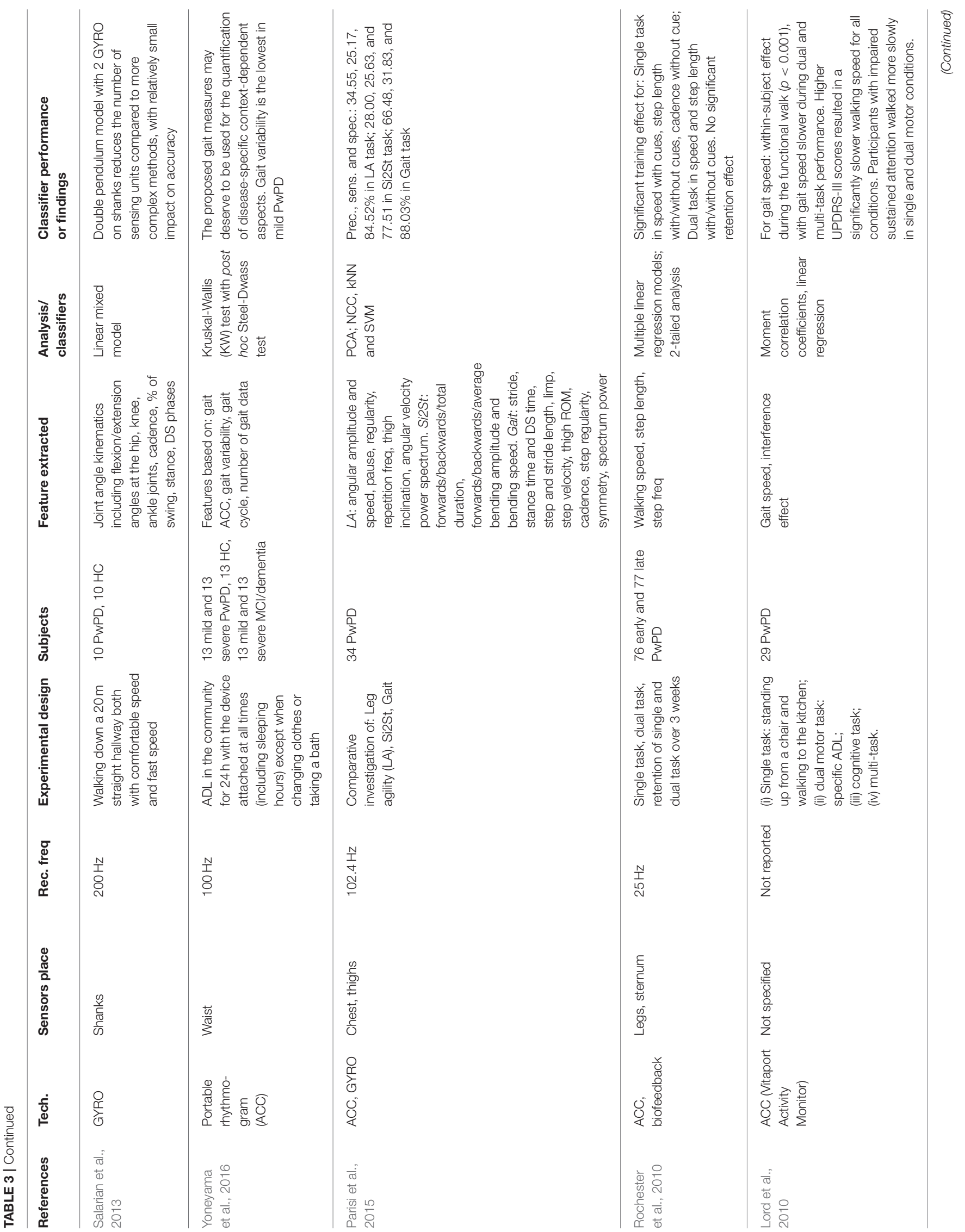




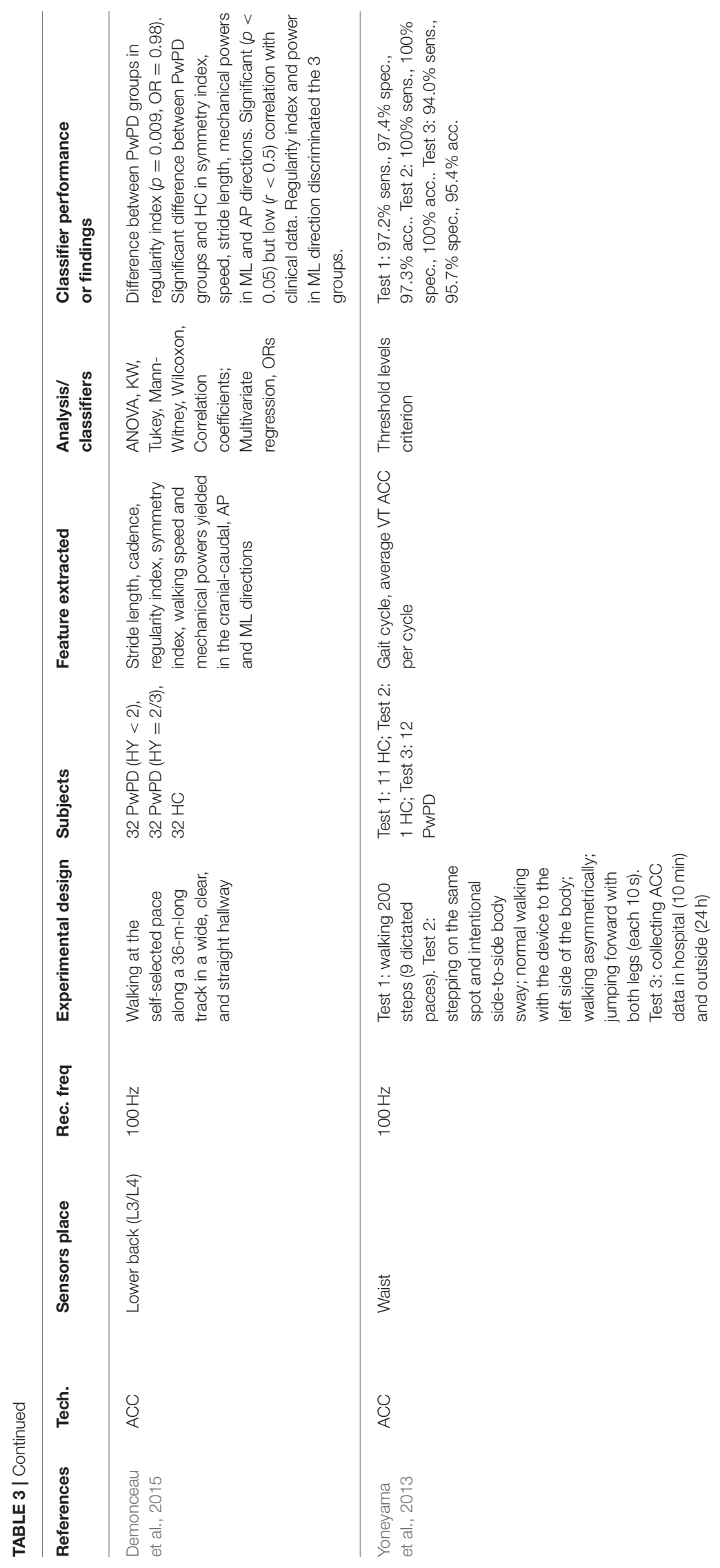




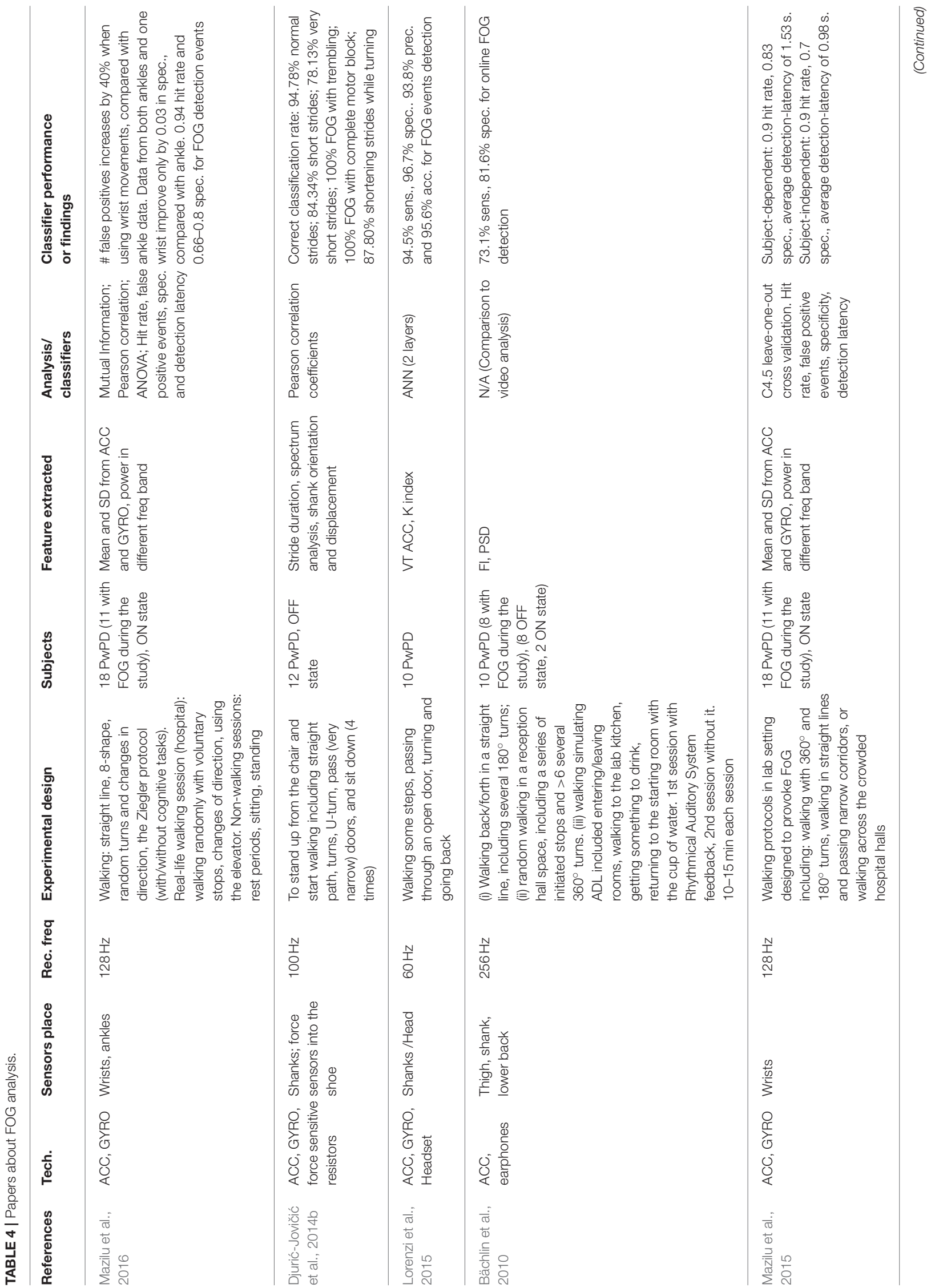




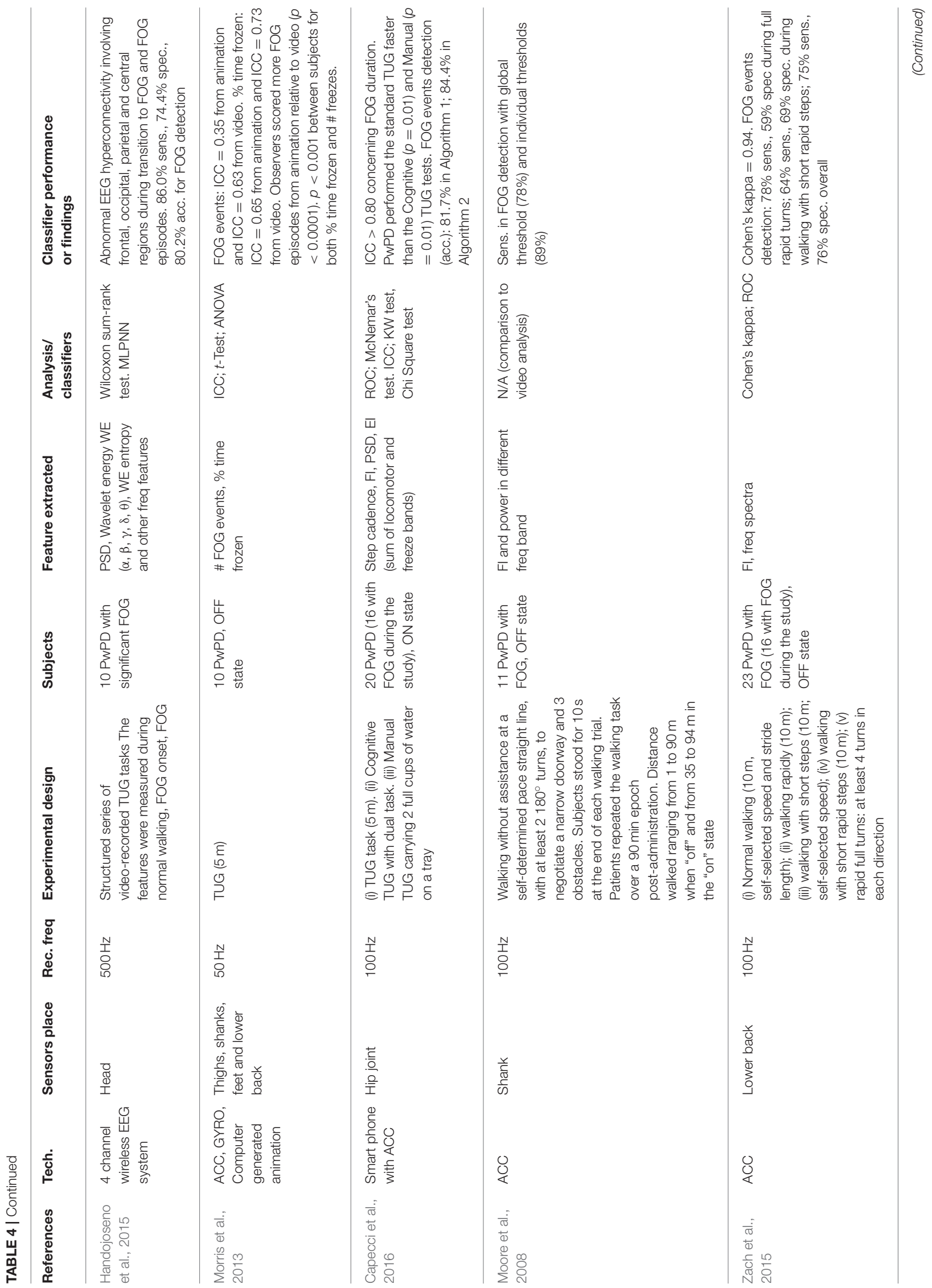




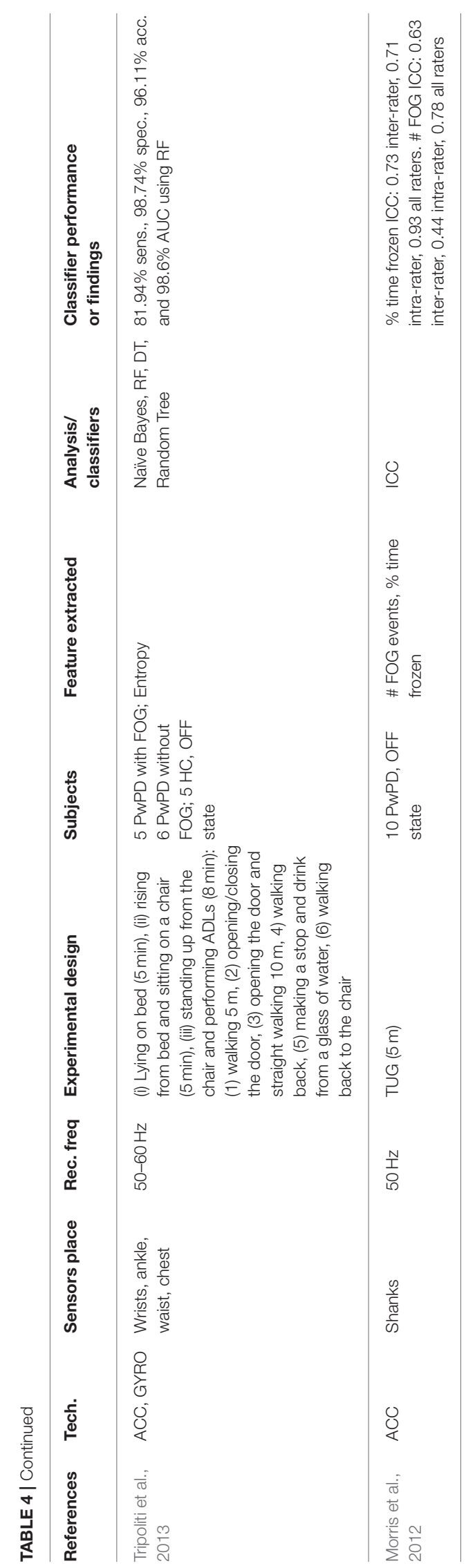

of the body (e.g., ankles, shanks, lower back) to measure the tremor in PwPD (Table 2). Further works proposed alternative solutions, such as smart clothes (Niazmand et al., 2011) or wearable glove systems, but the datasets for the experiments with these gloves were limited ( $\leq 5 \mathrm{PwPD}$ ) so were not analyzed in this review. In contrast, Bazgir et al. (2015), Kostikis et al. (2015), and Daneault et al. (2012) proposed smartphone-based systems that were mounted in a custom-made glove-case from which the acceleration signal was recorded. Finally, Braybrook et al. (2016) proposed the Parkinson's Kinetigraph System, a wrist-worn device able to collect data continuously over an extended time period and detect tremor events. Data for tremor analysis were collected while the subjects performed standard diagnostic exercises according to UPDRS for assessment of RT, PT, KT; or conducted a reaching task (Alhamid et al., 2010) or an action task (Kwon et al., 2016). In these exercises, it is crucial to recognize different typos of tremors (e.g., essential, resting, postural, re-emergent) accurately (Thanawattano et al., 2015; Surangsrirat et al., 2016), analyse the various frequency bands properly (Hossen et al., 2010, 2013; Niazmand et al., 2011; Daneault et al., 2012; Hossen, 2012; Rigas et al., 2012, 2016; Pierleoni et al., 2014; Bazgir et al., 2015; Ghassemi et al., 2016; Kwon et al., 2016; Mailankody et al., 2016; Zhou et al., 2016), and distinguish tremor correctly from other movements and disorders (e.g., dyskinesias, bradykinesia) (Salarian et al., 2007b; Rigas et al., 2012, 2016; Pierleoni et al., 2014), as well as recognize tremor severity accurately (Salarian et al., 2007b; Daneault et al., 2012; Rigas et al., 2012; Pierleoni et al., 2014; Bazgir et al., 2015). For these purposes, a frequency analysis was the most appropriate approach (Salarian et al., 2007b; Daneault et al., 2012; Hossen, 2012; Cavallo et al., 2013; Scanlon et al., 2013; Pierleoni et al., 2014; Bazgir et al., 2015; Braybrook et al., 2016; Zhou et al., 2016), and subjects with tremors in their hands were expected to have higher power in the high-frequency components (Alhamid et al., 2010). Although most of the works used signals from accelerometers to calculate features for tremor assessment, Surangsrirat et al. (2016) and Thanawattano et al. (2015) proposed the use of angular velocities to calculate the ratio of temporal fluctuations of tremor signal during resting tasks and kinetic tasks. This method can differentiate between PD tremor and ET since PwPD have a potential for higher tremor fluctuations with PD tremors. Additionally, Salarian et al. (2007b) used only gyroscope signals to calculate mobility and activity of the hand during selected time windows.

The principal aim of these works was to find a correlation between the features measured and the clinical scores assigned by the neurologists during medical examinations. Good results for correlation were achieved in several works (Pierleoni et al., 2014), which primarily used Pearson's coefficient (Salarian et al., 2007b; Cavallo et al., 2013; Kostikis et al., 2015; Rigas et al., 2016) and Artificial Neural Network (ANN) (Bazgir et al., 2015). Several machine learning approaches, including Support Vector Machine (SVM) classifier and Random Forest (RF), were implemented to predict the severity of tremor symptom (Kostikis et al., 2015). SVM (Hossen, 2012; Ghassemi et al., 2016; Surangsrirat et al., 2016), ANN (Hossen, 2012), combined Hidden Markov Model (HMM) (Rigas et al., 2012) Linear Discriminant Analysis (LDA), 


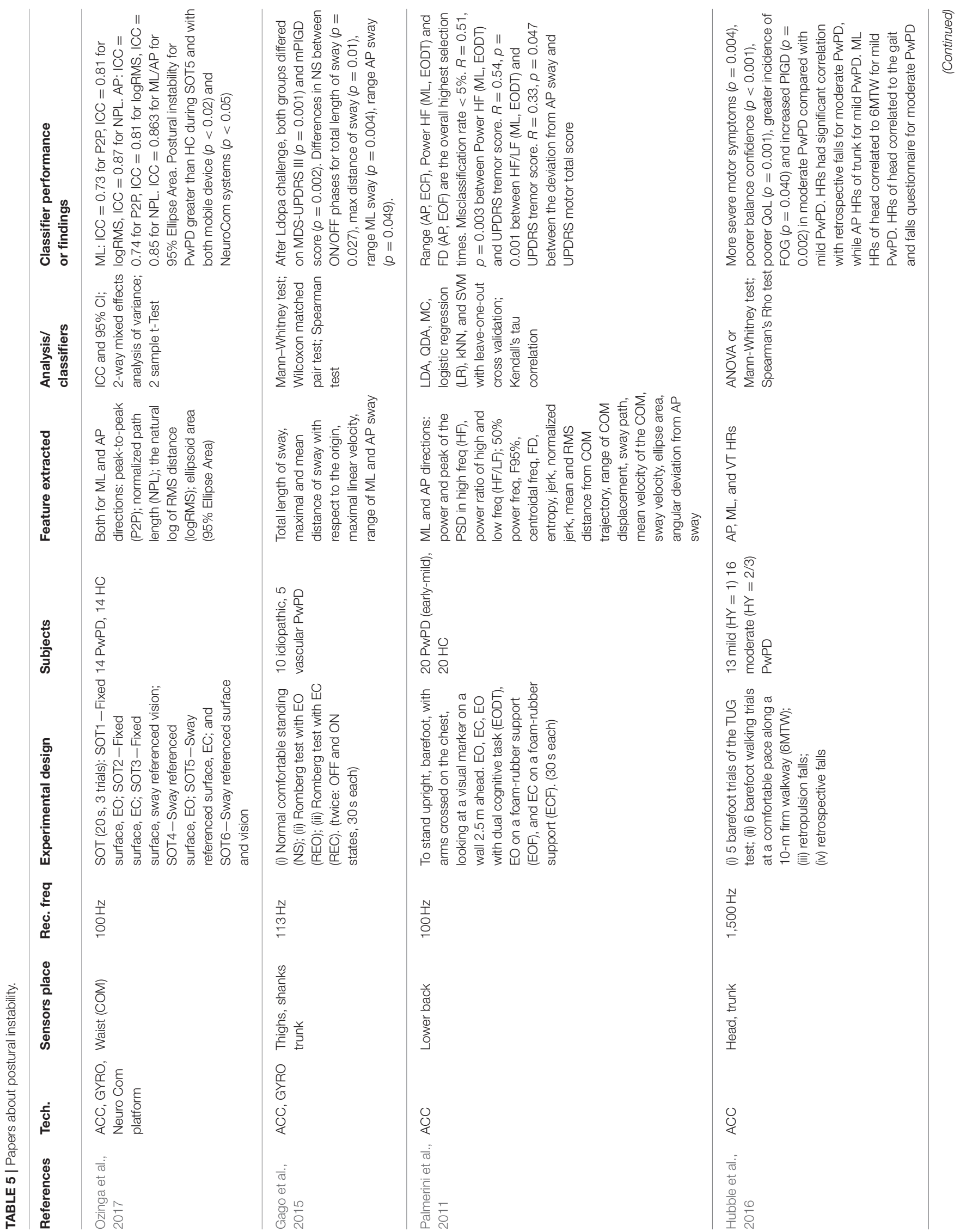




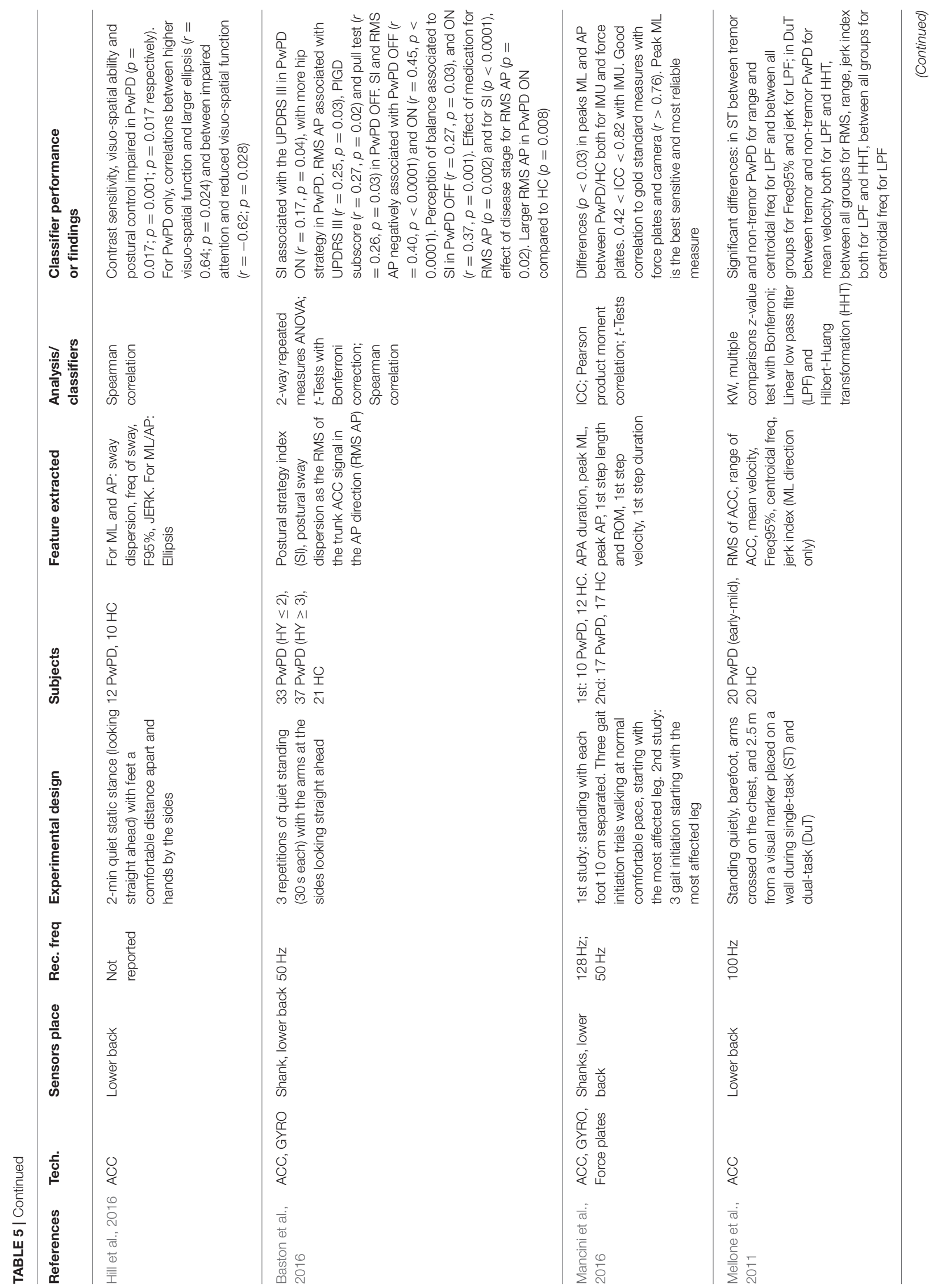




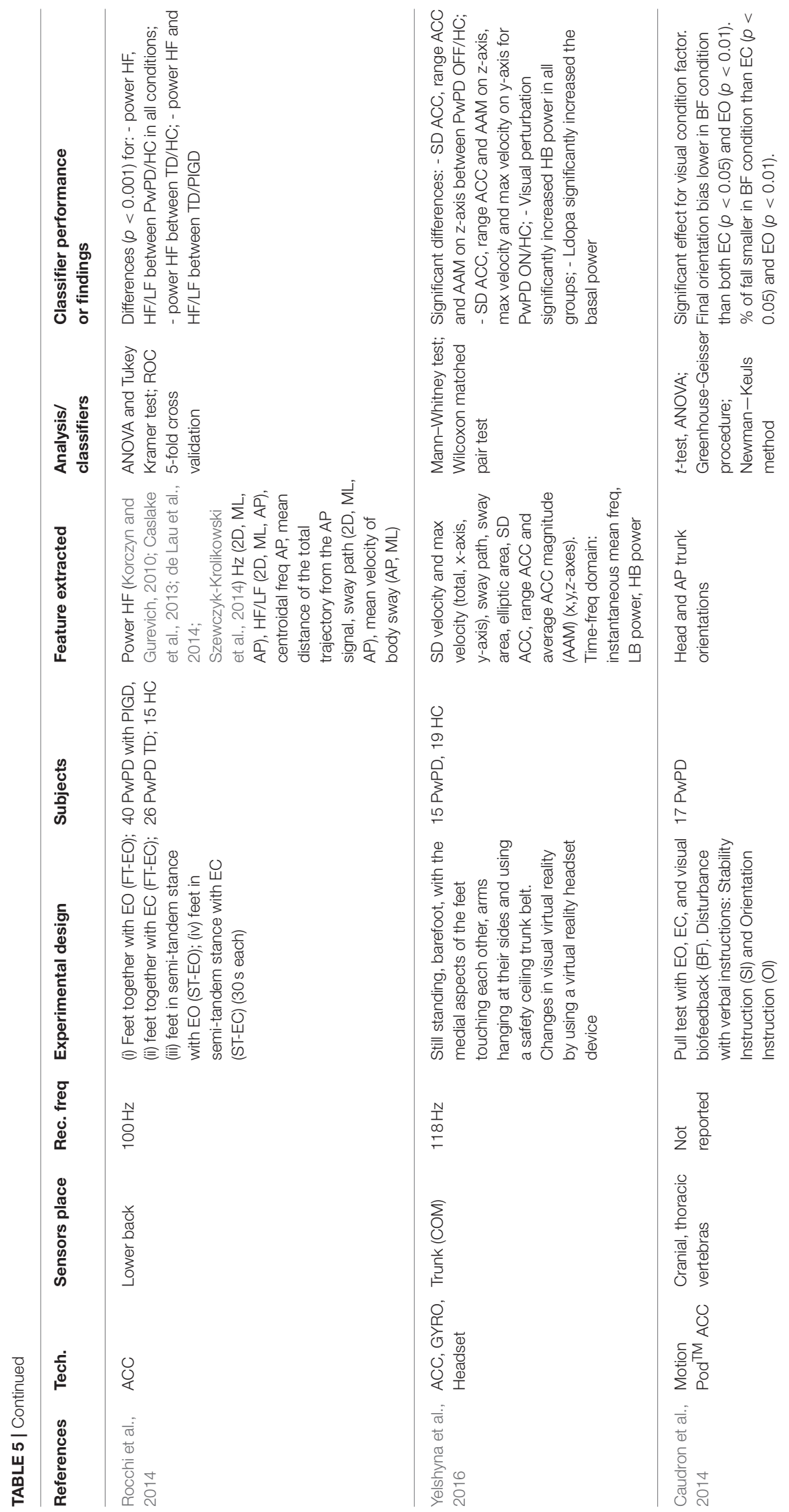




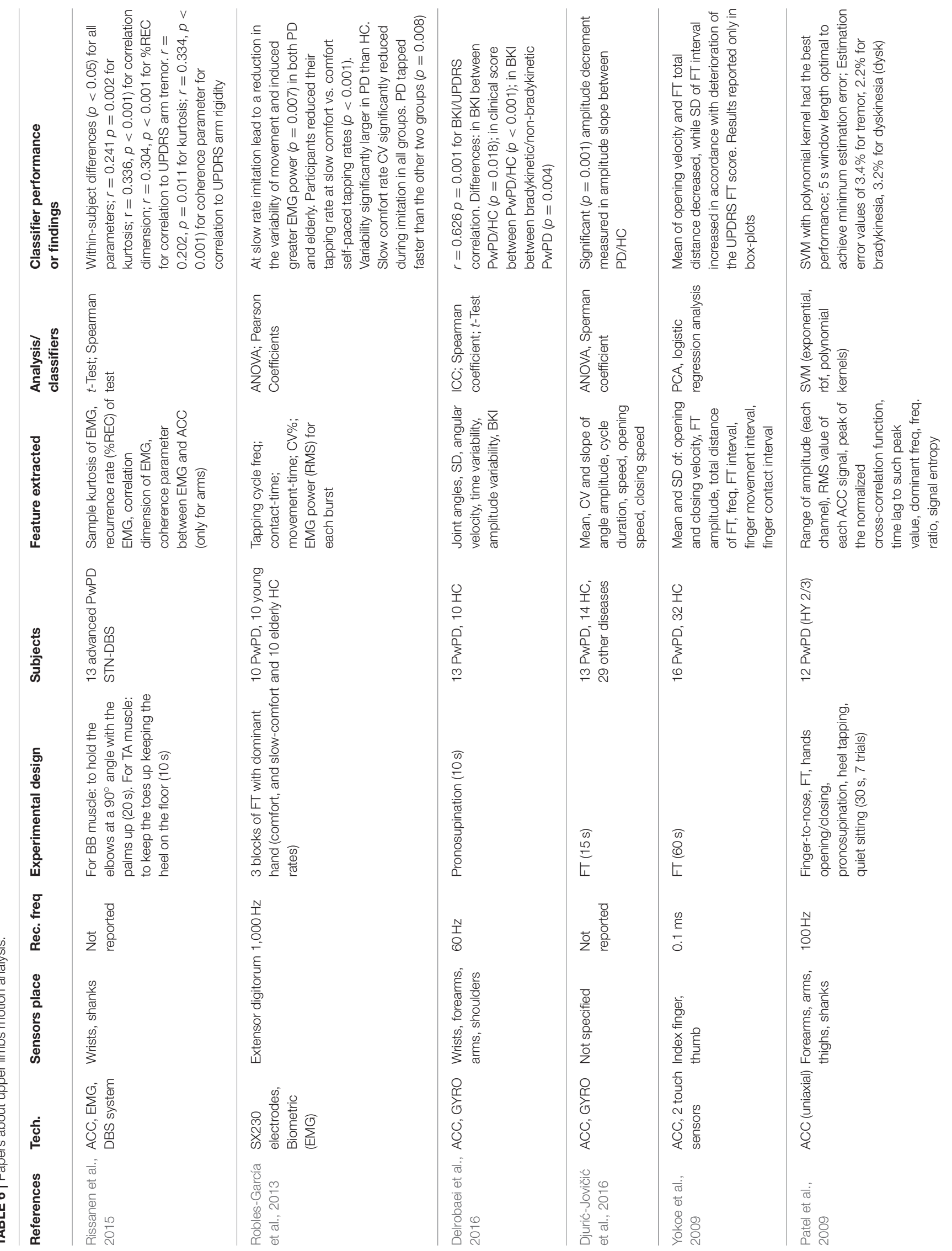




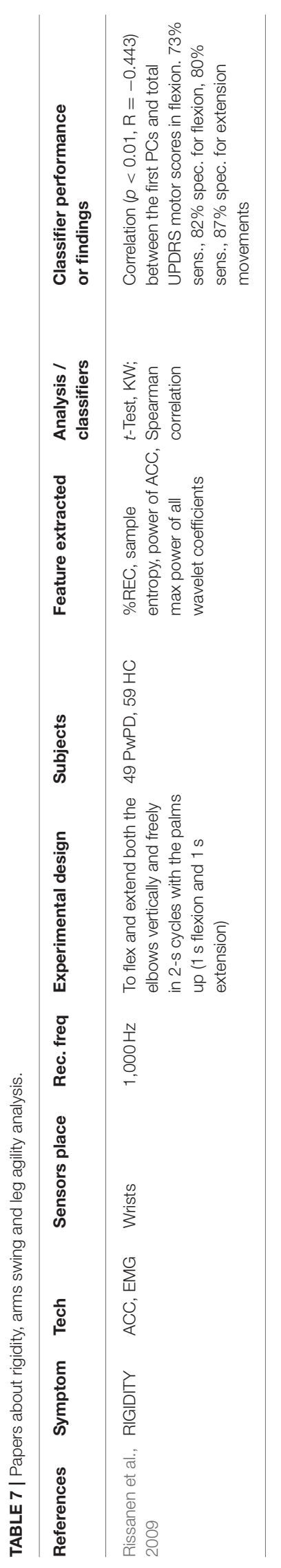

and $k$-means clustering (Ruonala et al., 2014a) were also used to distinguish between different groups of people (e.g., PwPD and HC, Parkinsonian and subjects with ET, or tremor PwPD and PwPD with other motor symptoms).

An alternative application was proposed by Hwang et al. (2009), who analyzed whether a light load can suppress tremor in the distal body segments. They demonstrated that in a PwPD the tremor is not suppressed, but actually it is enhanced in the proximal segments. They speculated that the application of greater inertial loads could reduce the tremor but could also be dangerous for patients that presented difficulties in balance and in postural strategies differently from healthy people. A load was also used by Ruonala et al. (2014a) who demonstrated that increasing the load to 1 or $2 \mathrm{~kg}$ decreased the accuracy in discrimination between PwPD, HC, and ET subjects.

From a different perspective, Fukumoto (2014) studied the effect of Ldopa treatment on tremor symptoms and found an increase of mean frequency and decrease of tremor power except for PwPD affected by motor fluctuations. Additionally, they found that visual and sound cues on tremor PwPD are able to improve tremor symptoms, similarly to the pharmacological therapy, although the Ldopa effect is most effective.

\section{Recommendations and Trends}

According to Zhou et al. (2016), the harmonics of real tremor are not sinusoidal, as those studied in some works to simulate or control the PD tremor, but they vary over time. Thus, papers in which only tremor is simulated were not included in the review. For this reason, inclusion of a large number of PwPD who are significantly affected by tremor is critical to test the efficacy of the proposed systems in measuring the severity of the symptoms (Ghassemi et al., 2016). Indeed, even though some papers provided for the recognition of tremor severity, difficulties remain in distinguishing between adjacent levels to define the correct stage of pathology (Rigas et al., 2012). In addition, the discrimination between patients with similar symptoms but different pathologies (i.e., Parkinsonian tremor and ET) (Hossen, 2012; Hossen et al., 2013) is not easy to achieve, but it is crucial for a correct diagnosis and treatment of the disease (Hossen, 2012; Thanawattano et al., 2015; Ghassemi et al., 2016; Surangsrirat et al., 2016). For example, although ET patients have a tremor that is dominant during action and posture tasks, while PD patients particularly have tremor during rest, it is not a simple matter to find features that discriminate well between the two groups. However, power spectral density seemed to be a good measurement (Hossen, 2012; Pierleoni et al., 2014).

From a technical point of view, uniaxial accelerometers are not sufficient to adequately analyse the motion, whereas the use of triaxial inertial sensors can provide a more detailed investigation regarding tremor detection. Tremors in hands vary from one person to another and may occur more in specific axes rather than others (Salarian et al., 2007b; Alhamid et al., 2010; Thanawattano et al., 2015). Also, the integration of IMU with other typos of sensors, such as EMG, can improve the accuracy (Hossen et al., 2010; Hossen, 2012; Ruonala et al., 2014a,b; Kwon et al., 2016; Mailankody et al., 2016) and the range of the measurements, so additional different typologies of 

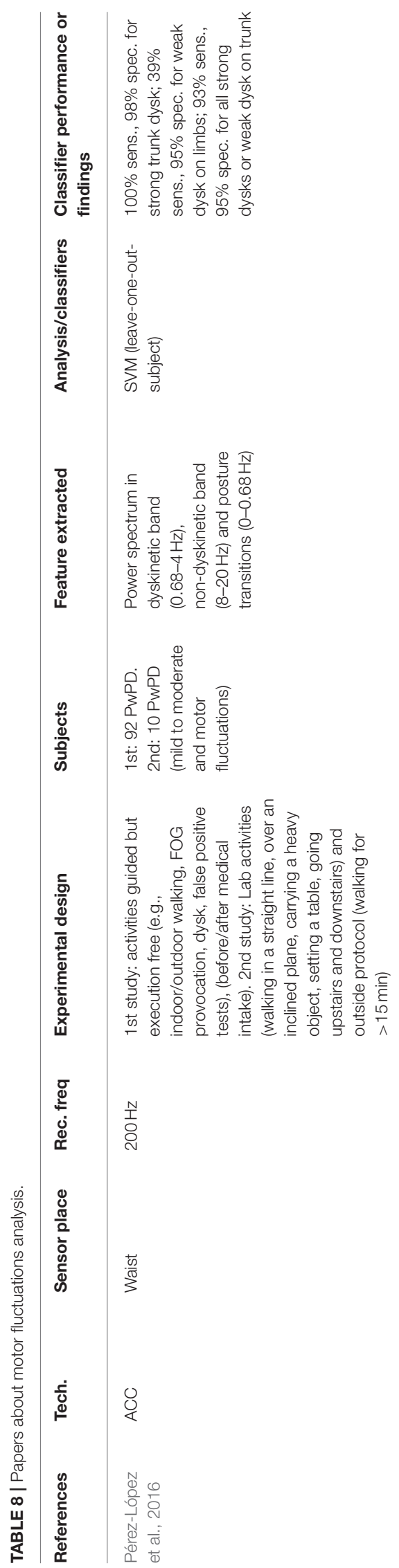

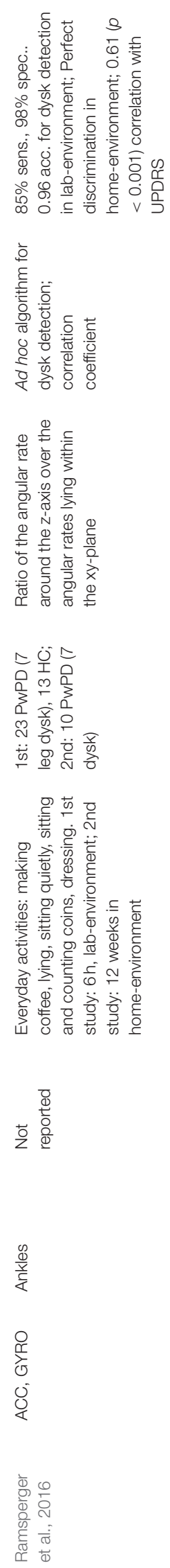

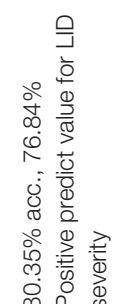

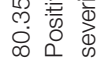
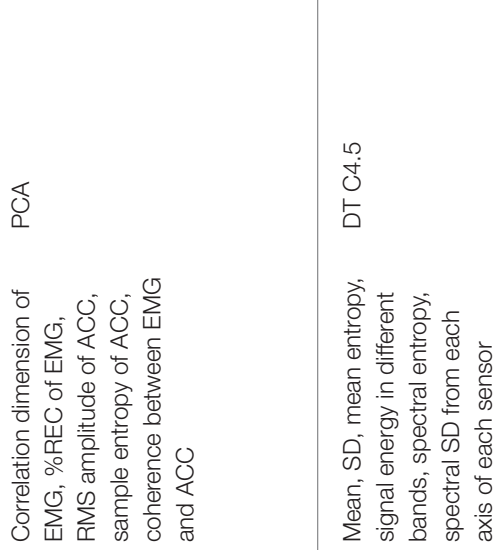

0
0
0
$\vdots$
0
0
0
0
0
0

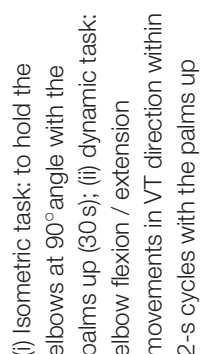

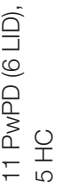

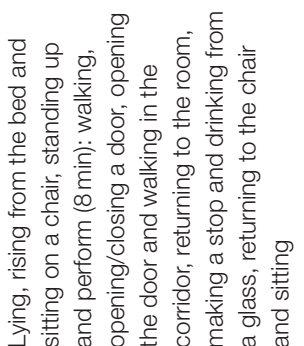

N
No
Oị

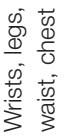

0
0
0
0
0
0

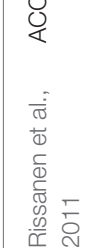

苍

$\underset{3}{\frac{\infty}{00}}$

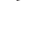

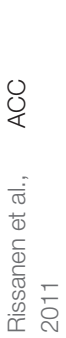



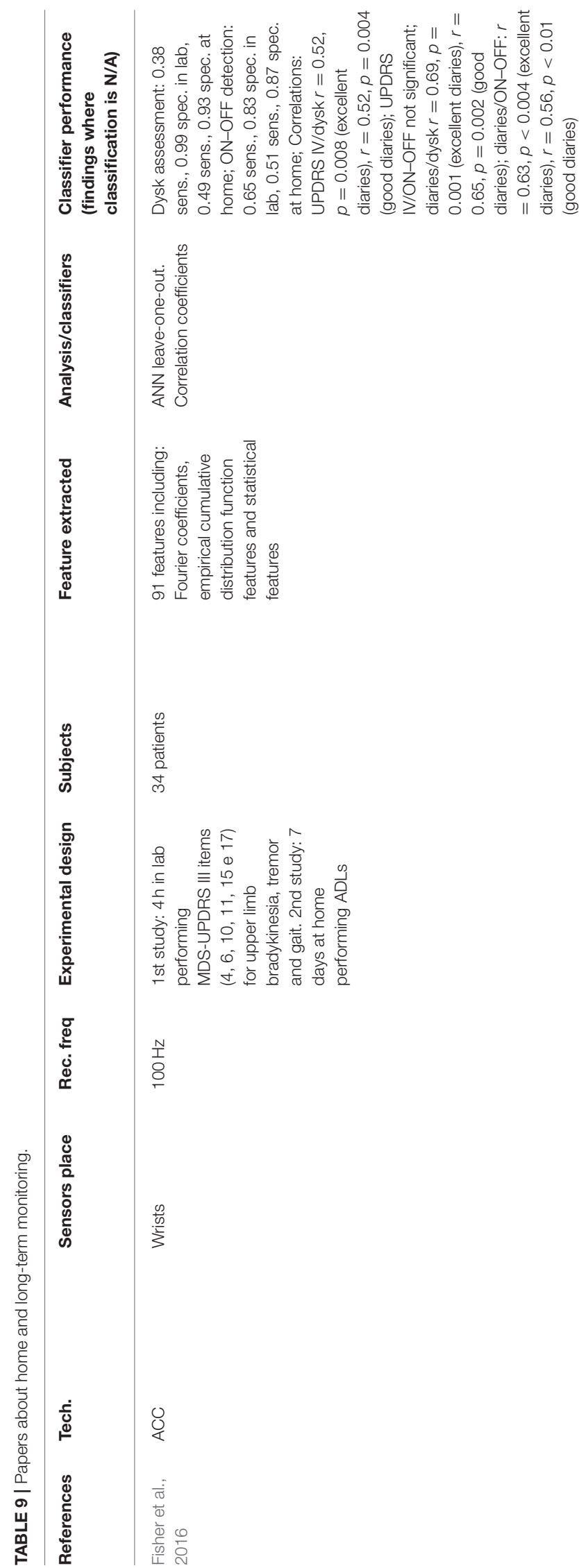

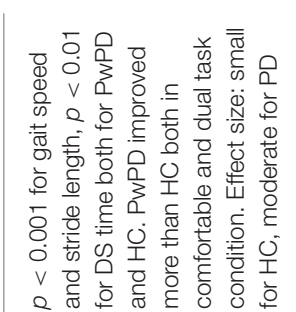

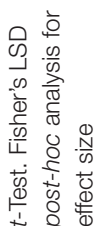

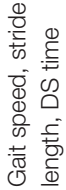

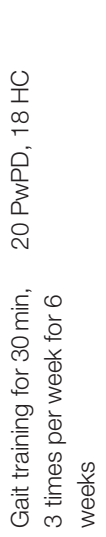

$\underset{\substack{1 \\ 8}}{\stackrel{1}{0}}$

운

$\mathscr{0}$
$\frac{8}{2}$
$\frac{8}{5}$
$\overline{0}$
0
0
0
0
0
0

है

c)

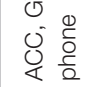

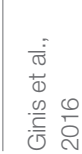

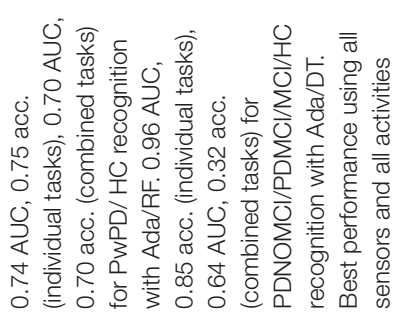

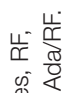

离

:

垈这

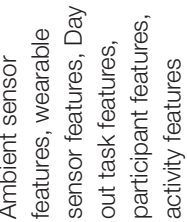

웅 오웅

is $\sum_{0}^{\infty}$

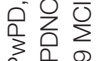

年

芦䓂高

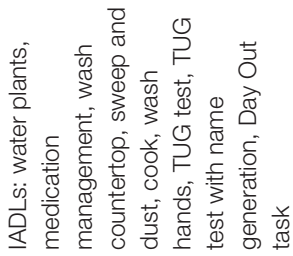

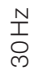

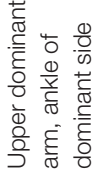

告

ठิ क

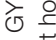

ن

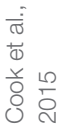

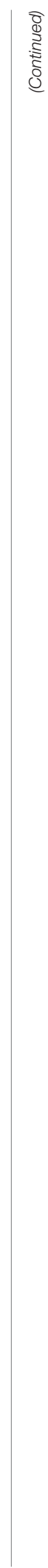




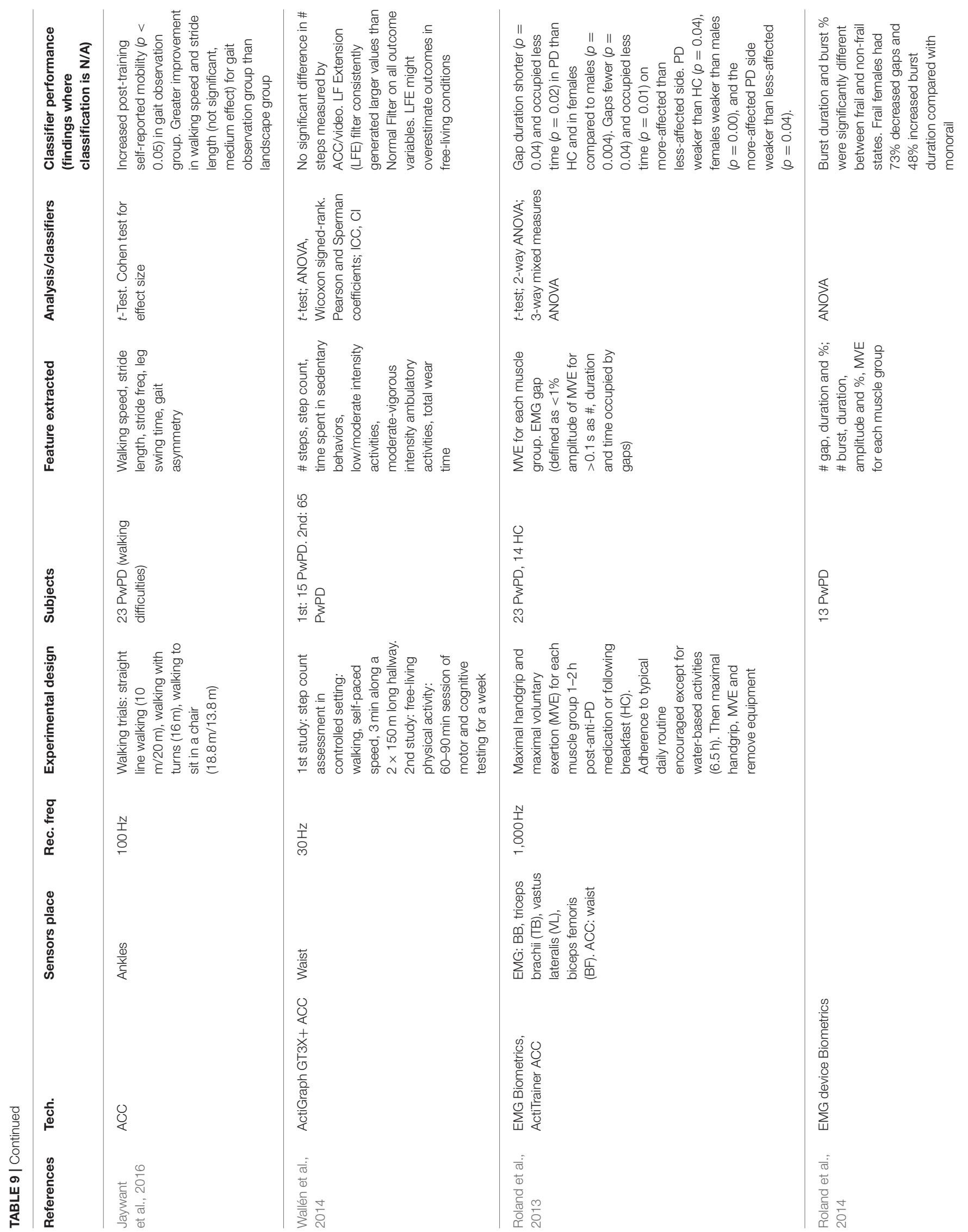




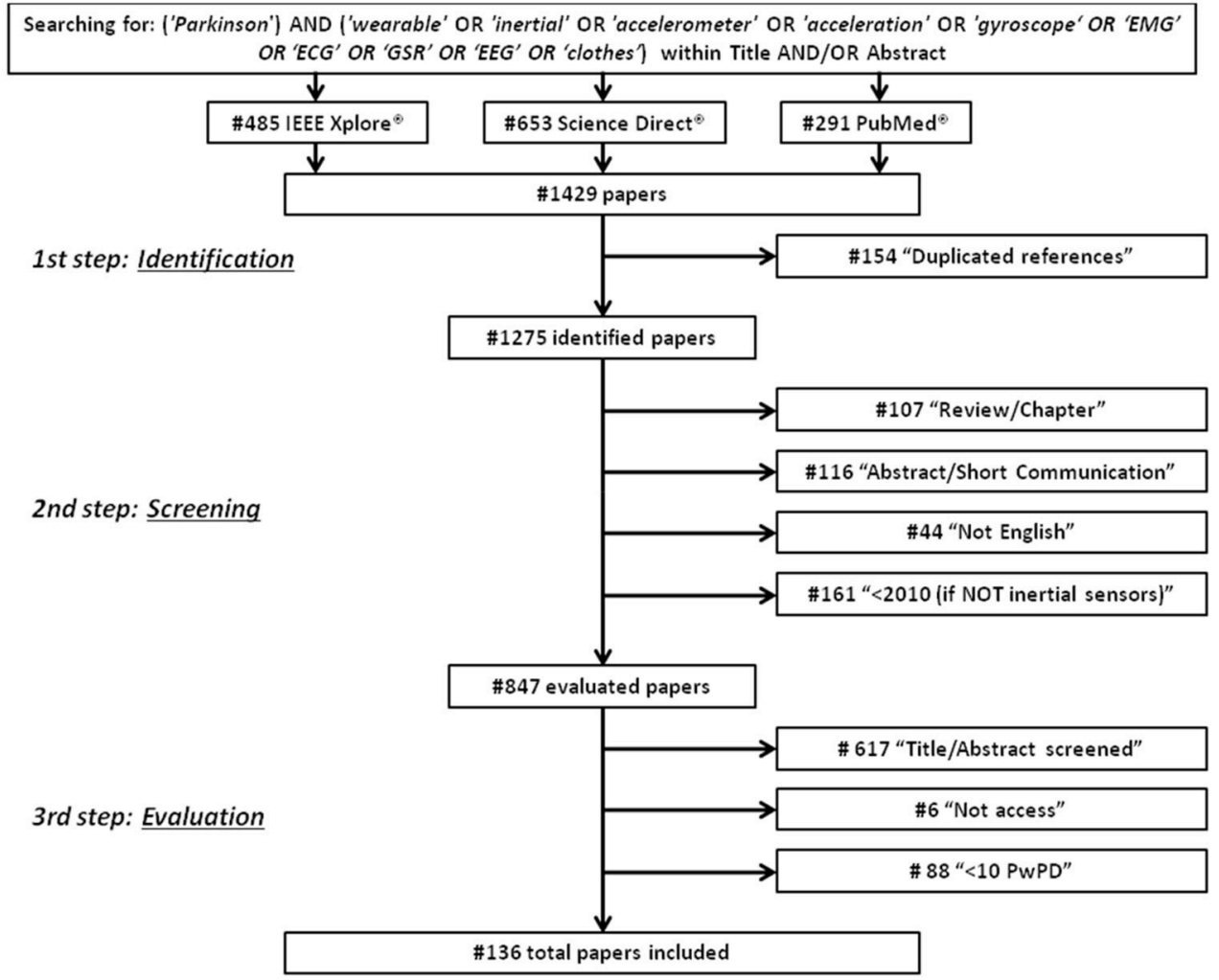

FIGURE 1 | Research methodology for review process.

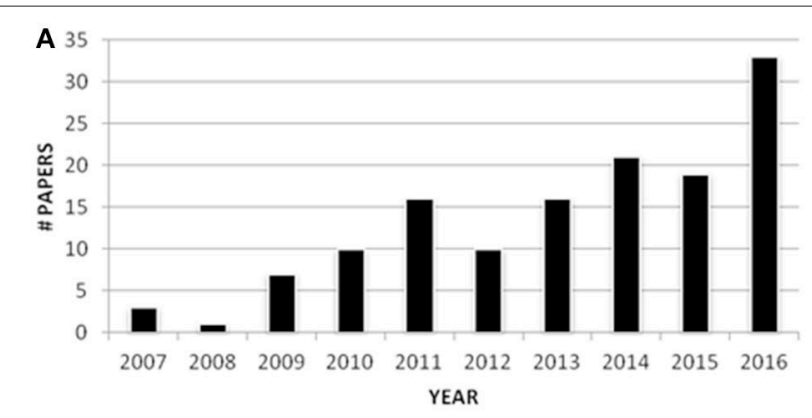

C

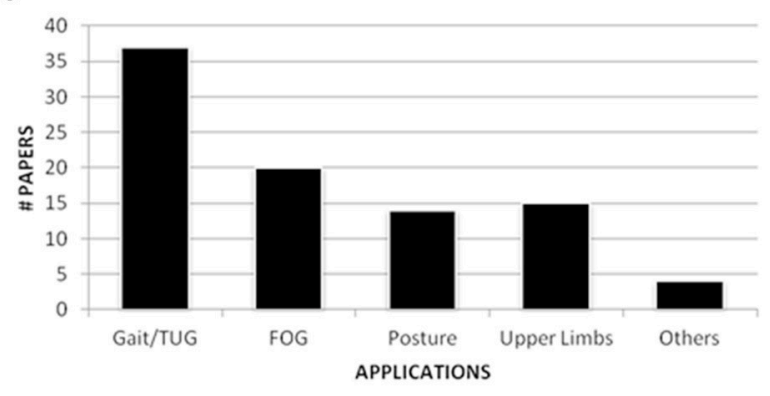

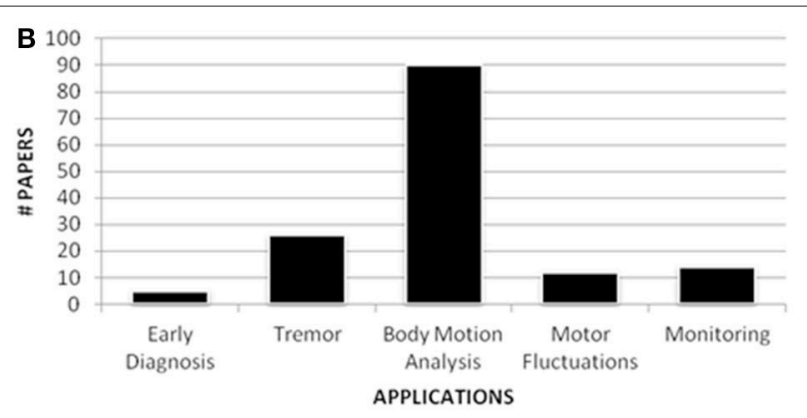

D

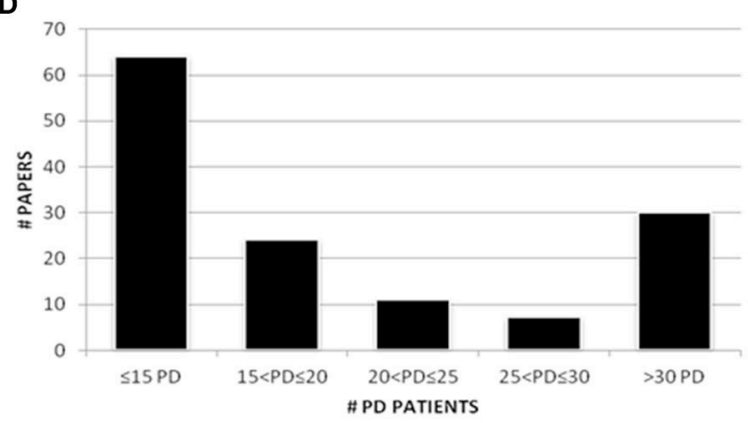

FIGURE 2 | Publication trend per year (A); paper distribution per application (B); papers within body motion analysis application (C); PD patients involved in the studies (D). 
sensors could be investigated with the goal of improving tremor recognition. Since the intra-individual variability of RT and PT frequency in the dexterity-dominant lower limb was lower in PwPD than in HC, and RT frequency differed between upper and lower limbs in PD, devices able to identify minute variations which are imperceptible upon expert clinical exam can be used to differentiate a diseased person from a healthy one (Scanlon et al., 2013).

Regarding the wearability of the devices, the use of gloves in which to insert the sensors does not seem to be an optimal solution because of the disadvantages due to the noises caused by the electronic parts and the discomfort related to the device, which is not adaptable to different hand sizes. On this topic, the solution proposed by Cavallo et al. (2013) seems to be promising in terms of wearability, portability, light weight, performance, and ease of use. In addition, the wrist-worn Parkinson's Kinetigraph System can accurately detect tremor over an extended time (Braybrook et al., 2016). Also, smartphone-based solutions (Daneault et al., 2012; Bazgir et al., 2015; Kostikis et al., 2015) could be an alternative for the measurement of tremor, even if the need to have a custom-made glove-case makes the device usable for only a short time. Additionally, the RT is identified consistently, whereas the measured PT correlates weakly with the clinical assessment, likely because the mass of the smartphone affected the dynamics of the hand/arm system (Kostikis et al., 2015). Added benefits of smartphone use are the common availability and the fact that smartphones do not require downloads or memory-consuming installation because the service provided is web-based. Further, the use of smartphones can provide for a ubiquitous assessment of the disease both in the clinical setting and the home environment. Finally, smart clothes can represent an additional solution that is comfortable to wear and records data independently from a laboratory or technical staff for long-term monitoring (Niazmand et al., 2011).

The systems must be portable and lightweight to avoid disturbing the characteristics of the tremor; capable of being mounted to a predetermined anatomical anchor point (Alhamid et al., 2010; Cavallo et al., 2013); and able to provide timely feedback to the users. Thus, a wired system (i.e., connected by USB cable) to implement an offline analysis or a prototype of large dimensions (Salarian et al., 2007b) must be overcome in favor of totally wireless devices equipped with algorithms for realtime data analysis able to process the tremor quantification and prediction models. For this purpose, dynamic algorithms and models that allow the examination of the time-varying nature of tremors (Rigas et al., 2012) in the presence of unscripted and unconstrained voluntary movements (Cole et al., 2011; Roy et al., 2011) could be a valid solution. Tremor suppression is another important problem to improve QoL in PwPD who suffer with this symptom, and devices able to accomplish this must be investigated and implemented. For this purpose, Zhou et al. (2016) obtained crucial information in their recent study. They affirmed that PD tremor is composed of multiple harmonics with time-varying amplitude; thus, it is not a monofrequency vibration. In particular, the $2 \mathrm{nd}$ and the $3 \mathrm{rd}$ harmonics are so strong that they cannot be neglected. Ignoring these components could lead to development of inefficient tremorsuppression devices. This phenomenon of harmonic peaks in higher-amplitude tremors carries also to differential diagnostic information when different typos of tremors must be recognized (e.g., Parkinsonian tremor and ET) (Hossen et al., 2010).

\section{Application 3: Body Motion Analysis}

The cardinal features of PD are tremor, postural instability, muscular rigidity, and bradykinesia and/or hypokinesia. Thus, PD patients are characterized by a worsening of the motor performance that can be very disabling for them. These symptoms appear evident in different parts of the body, such as trunk, and lower and upper limbs. Generally, the symptoms are assessed by the neurologist during medical examination through visual inspection, in which the patients are asked to perform typical tasks described in the motor section of MDS-UPDRS (MDS-UPDRS III). In particular, for lower limbs the most investigated tasks are gait, including the disabling common complication known as freezing of gait (FOG), and the Timed Up and Go (TUG) test. For upper limbs, the research focused on finger tapping, alternating hand movement, pronation/supination, and finger-to-nose movement. Only one work concerning a multimodal system able to analyse motor tasks from both upper and lower limbs was found (Oung et al., 2015). Totally, 90 papers were assessed within this application. Considering the wide range of impairments related to the body motion, this application area is divided into five sub-sections, concerning different body segments or symptoms, which are named: gait and TUG test, freezing of gait, postural instability, upper limbs and other symptoms (leg agility, rigidity, and arms swing).

\section{Gait and Timed Up and Go (TUG) Test}

Gait is the most examined task in the studies for the analysis of motor performance in PwPD (37 papers were included). Motion capture systems (e.g., ultrasound system, optical system, and/or forceplates) are the gold standard for motion analysis. These systems are typically used to assess the parameters characterizing gait, but they are expensive, unportable, and usable only in laboratory environments. Recent studies also support the use of IMUs to assess objectively the movement of PwPD by demonstrating the validity of IMUs in comparison to motion capture systems (Del Din et al., 2016; Ferrari et al., 2016; Sejdić et al., 2016). Several studies showed the use of accelerometers (Stamatakis et al., 2011; Palmerini et al., 2013; Jarchi et al., 2015; Del Din et al., 2016; Sejdić et al., 2016), gyroscopes (Fatmehsari and Bahrami, 2010; Grimpampi et al., 2013), or both methods (Oung et al., 2015; Trojaniello et al., 2015; Ferrari et al., 2016), placed on different segments of the body (e.g., shank, thigh, foot, lower back) to measure the performance of gait in PD patients, in particular to assess both TUG test and longdistance walking, to distinguish between $\mathrm{HC}$ and $\mathrm{PwPD}$ during specific tasks (Esser et al., 2013; Mariani et al., 2013; Del Din et al., 2016; Table 3). An alternate approach foresees the use of a smartphone-equipped triaxial accelerometer (Arora et al., 2014) or a StepWatch worn on the wrist (Schmidt et al., 2011) to capture the movement of patients during preset gait tests. 
Statistical (e.g., mean, variance, skewness, kurtosis), frequency (e.g., energy, power spectral density, fundamental frequency), and spatiotemporal/kinematic (e.g., stride length, TUG time, stride velocity) features were extracted and analyzed. Step or stride segmentation were key points for the gait analysis to recognize heel-strike and toe-off times characterizing the gait cycle and the complete walk (Barth et al., 2013; Del Din et al., 2016; Parisi et al., 2016). The experimental protocols were principally based on TUG exercise and gait. The TUG test consisted of standing up from the chair and walking a $3 \mathrm{~m}$ (or $7 \mathrm{~m}$ ) distance at a normal speed, followed by a turn of $180^{\circ}$ and walking back, and ending with another turn of $180^{\circ}$ and sitting down on the chair (Salarian et al., 2010; Weiss et al., 2010; AlJawad et al., 2012; Mariani et al., 2013; Palmerini et al., 2013; Reinfelder et al., 2015). Restricted sit-to-stand (Si2St) task with feet fixed on the floor without any linear translation movement (Giuberti et al., 2015); extended TUG test (ETUG) with $10 \mathrm{~m}$ to walk and wide curve trajectory (Caldara et al., 2014); and Instrumented Stand and Walk Test (ISAW), which is a TUG in which the phase of standing up and sitting down are not included (Curtze et al., 2016; Horak et al., 2016), are variations on the traditional tasks. Alternatively, other works focused on gait tests on short (Esser et al., 2013), moderate (Schmidt et al., 2011; Arora et al., 2014), and long distance (Weiss et al., 2011), including $180^{\circ}$ turns (Mariani et al., 2013; Rahimi et al., 2014); or straight walking at different speeds (e.g., comfortable, slow, fast) (Salarian et al., 2013; Del Din et al., 2016); or random walking with initiated stops and several $360^{\circ}$ turns; or basic mobility-related activities (e.g., lying, standing) and domestic activities (Barth et al., 2013; Yoneyama et al., 2016). Only Barth et al. (2011) and Oung et al. (2015) proposed to analyse exercises able to assess foot mobility (e.g., heel-toe tapping or foot rotation), whereas, Parisi et al. (2015) proposed a comparative outlook of different tasks: gait, sit-to-stand, and leg agility. Others (Lord et al., 2010; Rochester et al., 2010) implemented experimental protocols which include single, dual, and multiple tasks to analyse the effect of external cues on gait strategies. Only one work (Salarian et al., 2009) was focused on turning; it recognized differences between early PwPD and HC with excellent sensitivity and reliability thanks to the automatic detection of all turns. The majority of the works compared the performances of a group composed of PwPD and a group of control subjects (Barth et al., 2013; Palmerini et al., 2013; Arora et al., 2014; Oung et al., 2015; Parisi et al., 2015), and showed that the second group had better results in terms of time of execution, speed (Horak et al., 2016), regularity, cadence, symmetry, stride length (Demonceau et al., 2015), amplitude, and slope (Weiss et al., 2011). Others implemented multi-class classification to distinguish among $\mathrm{HC}, \mathrm{PwPD}$ without gait disturbance, and PwPD with gait disturbance (Tien et al., 2010) or compared the performance of $\mathrm{HC}, \mathrm{PwPD}$, and subjects with dementia (Yoneyama et al., 2016). Moreover, recent European research projects, including REMPARK (Cabestany et al., 2013), PERFORM (Cancela et al., 2011), and CuPiD (Ferrari et al., 2016), used systems based on wearable IMUs to examine disease management and assessment with artificial intelligence and to try to identify the gait and movement of PD disorders.

\section{Recommendations and trends}

As with other applications previously analyzed, some works presented limited datasets, investigating groups that were not age-matched (Ferrari et al., 2016) and sometimes including other pathologies in addition to PD (Schmidt et al., 2011; Salarian et al., 2013). Thus, bigger sample sizes are needed to confirm the significance of the novel gait parameters (Mariani et al., 2013). In several studies, moderate patients (e.g., $H Y=2 / 3$, Palmerini et al., 2013; Yoneyama et al., 2013; Sejdić et al., 2016) were involved, so the difference in performance between $\mathrm{PwPD}$ and $\mathrm{HC}$ are easily identifiable. The recruitment of PwPD in the first stage of the disease (i.e., $\mathrm{HY}=1$ ) should be primarily investigated to demonstrate the accuracy and the objectivity of the technological solutions with respect to the traditional clinical evaluations (Demonceau et al., 2015), aiming to achieve early diagnosis of the pathology (Barth et al., 2011). Many gait analysis protocols have been developed to complete the medical exam of PD patients, but the optimal method remains under debate (Demonceau et al., 2015). Regardless, the use of inertial sensors placed on different parts of the body seems to be a promising method for objective estimation of the parameters of the gait (Barth et al., 2013; Del Din et al., 2016; Ferrari et al., 2016). The performance of the inertial sensors is different from that of commercial pedometers that are less accurate in quantifying gait performances. Although the TUG test, which includes turning movements, was analyzed in several papers, and the importance to classify the different phases with the TUG test is recognized (Reinfelder et al., 2015), only one work specifically dealt with the rotation task. This is likely because turning is not directly measured in UPDRS, and the PIGD sub-score as a clinical measure of reference has limited compliance (Salarian et al., 2009). The majority of the papers aimed to distinguish between the PwPD and HC and compared the spatiotemporal and frequency features measured during the protocol adopted. Only a restricted number of studies (e.g., Salarian et al., 2010, 2013; Tien et al., 2010; Parisi et al., 2015) focused on developing full biomechanics analysis to measure biomechanical parameters, such as joint range of motion, ankle dorsiflexion, finger flexion, etc., and investigated the benefit of using such kind of features in artificial intelligence algorithms. Within the same papers, different feature selection methods could be examined and compared; those revealing the best accuracy in distinguishing between the two groups were selected (e.g., SVM, LDA, RF, odds ratios (ORs), $\mathrm{k}$ nearest neighbors $(\mathrm{kNN})$, nearest centroid classifiers (NCC), $t$-test]. Parisi et al. (2015) also reported good results in correlating kinematic features and UPDRS scores, although the automatic system tends to underestimate the actual UPDRS scores. The lack of a meaningful correlation might be due to the relatively blunt nature of UPDRS in assessing PD symptoms (Yoneyama et al., 2013). Alternatively, Barth et al. (2013) implemented a step segmentation algorithm based on Dynamic Time Warping (DTW), which has the main advantage that the two input series do not need to be aligned in the time domain, and the error caused by the non-linear relation of the two series can be avoided. Other papers pointed out conflicting results, even when starting from identical hypotheses 
(Salarian et al., 2009; Palmerini et al., 2013). For Palmerini et al. (2013), in fact, temporal measures of PwPD are normal compared to $\mathrm{HC}$, but patients are characterized by reduced smoothness and dynamics in trunk movement during gait and turining. However, the misclassification rate of $22.5 \%$ in the early-mild stage of the disease is high. Further, the separation between PwPD and HC is not challenge anymore, because an accurate distinction within the group of PwPD should be achieved to assess the severity of the pathology at each moment (Barth et al., 2011). This task remains difficult, even though significant differences between mild and severe PwPD (Yoneyama et al., 2016), as well as between mild and moderate PwPD (Demonceau et al., 2015) were seen. Further, Parisi et al. (2015) revealed the contribution of the sit-to-stand task to distinguish between patients with slight and mild symptoms and those who manifest moderate or severe impairments. Finally, for the TUG test, accelerometer-derived parameters, in addition to test duration, could represent complementary and objective biomarkers of PD to assess the pathology progression and therapeutic response (Weiss et al., 2010). To minimize the invasiveness of the devices and to improve the acceptability of the systems proposed, it is important to try to reduce the number of sensor units using existing biomechanical models and place the devices in a way that does not interfere with gait (Salarian et al., 2013). Fatmehsari and Bahrami (2010), for example, demonstrated that a single gyroscope attached to either shank or thigh is sufficient to discriminate between PwPD and HC by calculating non-linear features. Trojaniello et al. (2015) confirmed that a single IMU placed on the lower back works well for healthy subjects, but it shows difficulties for impaired gait. This result was confirmed by Ferrari et al. (2016), who found that shuffling gait could mask the proper detection of initial contacts and foot-off events. In contrast, Sejdić et al. (2016) obtained good results in pathological subjects, as did Del Din et al. (2016), who affirmed that a single accelerometer on the lower back is sufficient for measuring gait characteristic, including asymmetry and variability. Therefore, in pathological situations, the use of sensors placed on both legs is recommended (Reinfelder et al., 2015) so that data from left and right sides can be merged for the final evaluation (Ferrari et al., 2016). A smartphone-based solution (Arora et al., 2014) or the StepWatch (Schmidt et al., 2011) can be innovative in terms of wearability because the sensors are hidden in common tools, and they can accurately count the strides. However, these solutions do not allow measurement of clinical features of interest such as stride length, so a complete analysis of the movement is not possible, and direct comparison with other systems is not feasible. For future implementations, it is crucial that the results of the gait analysis are shown immediately after execution of the test, through the development of semi-automated operations (Caldara et al., 2014) or dedicated applications available on smartphones (Ferrari et al., 2016), to enable real-time gait analysis. The algorithms should automatically detect all transitions and all turns, showing differences between $\mathrm{HC}$ and PwPD and good test-retest reliability (Salarian et al., 2010), even if large variations in results are common due to different walking styles. Although the principal aim of gait analysis is to quantify the motor performance of the patients to provide a more accurate diagnosis of the pathology, gait analysis can be associated with other applications, including rehabilitation, supporting decision-making (Grimpampi et al., 2013), biofeedback for gait monitoring, and fall prevention (Caldara et al., 2014). The use of adequate external cues can improve the gait stability for early/mild patients, but the cues become less effective for advanced patients. However, the use of auditory, visual, and somatosensory cues during single and dual tasks enhance motor learning in PwPD (Rochester et al., 2010), so they could reasonably support rehabilitation programs. Differently, the implementation of dual and multi tasks that measured selective, divided, and sustained attention, negatively interfered with the gait (Lord et al., 2010).

\section{Freezing of Gait}

FOG is one of the more disabling complications, especially in elderly long-term, advanced PwPD. Motor blocks are a subtype of the FOG phenomenon that primarily affect the gait initiation process. They include delayed release of anticipatory postural adjustments (APA), hypokinetic APA (reduced scaling), and bradykinetic APA (abnormal timing), suggesting the existence of a pathophysiological mechanism that involves both locomotor networks and cortical areas (Delval et al., 2014). FOG episodes mainly appear at the gait initiation, when the patient must turn or when to the patient must pass through narrow spaces. The gold standard for FOG evaluation is direct or video recorded gaiteven if, often, the FOG questionnaire (FOG-Q) is administered (Bächlin et al., 2009). Worsening coordination during gait is another feature characterizing PwPD, and is directly correlated to FOG severity (Mazilu et al., 2016). In the 20 studies included in this review, IMUs were used alone, with other sensors, or integrated in different technological devices to improve the detection of FOG events (Table 4). Force sensors (Djurić-Jovičić et al., 2014b), EMG (Cole et al., 2011), headsets (Lorenzi et al., 2015), earphones (Bächlin et al., 2009, 2010), ECG and Galvanic Skin Response (GSR) sensors (Mazilu et al., 2015), and a portable four-channel wireless electroencephalogram (EEG) system (Handojoseno et al., 2012, 2013, 2014, 2015) were the most common supplementary devices used to provide biofeedback. In contrast, Morris et al. (2013) proposed a validated method to assess the phenomenon using a computer-generated animation and reconstructed data coming from IMUs. Capecci et al. (2016) used a smartphone at the hip joint to record gait data to detect FOG events. Mazilu et al. (2016) proposed to apply IMU on the wrist since movement on the upper limbs is also highly correlated with FOG events, and the wrist seems to be a convenient place in terms of unobtrusiveness, usability, and acceptability. Furthermore, results from both ankles and wrist are minimally better than those obtained by ankles only. To define a parameter to assess FOG episodes, Moore et al. (2008) analyzed the frequency characteristics of vertical leg movement during walking. They introduced the Freeze Index (FI), the ratio between the power of the gait signal in the "freeze" band (3$8 \mathrm{~Hz})$ and the power in the "locomotor" band $(0.5-3 \mathrm{~Hz})$. When experiencing a freezing episode, a "trembling" of the leg was observed, reflected in the power spectra of vertical leg movement with high-frequency components in the band 2-6 Hz. Zach et al. 
(2015) also adopted the FI to identify freezing episodes by analyzing FOG-eliciting tasks as rapid full turns and walking with rapid short steps. They obtained low specificity due to false positive events detected by the sensor but not revealed by video analysis. Additional information about step cadence can improve sensitivity and specificity for FOG event recognition, avoiding false event detection (Capecci et al., 2016). Tripoliti et al. (2013) instead proposed to measure the entropy related to the freezing event, since it is a nonlinear parameter, as is the FOG disorder. Djurić-Jovičić et al. (2014b) proposed a novel method that used the Pearson's correlation coefficient to define the "representative stride" and the "normal zone" to separate normal from abnormal gait, distinguishing also between straight and turning strides. Alternatively, Cole et al. (2011) proposed a dynamic neural network to better capture the time-varying nature of FOG, because the method enabled them to learn how the features representative of FOG events can change over time. Differently, Handojoseno et al. performed frequency and wavelet analysis to extract significant features from EEG signals and then used MLPNN to detect FOG episodes with accuracy ranging between 70 and 80\% (Handojoseno et al., 2012, 2013, 2014, 2015). Vibration and auditory biofeedback methods were implemented (Bächlin et al., 2009, 2010; Mazilu et al., 2014) to provide a cue to alert the patient about the occurrence of a FOG event. This kind of intervention can be helpful in preventing falls due to FOG episodes, with the aim to reduce major complications for the PwPD both in terms of health and costs. To allow the biofeedback intervention and the alert to the patient, a real-time processing of data is needed (Bächlin et al., 2009; Mazilu and Hardegger, 2012; Mazilu et al., 2014). Recently, in European projects (e.g., REMPARK, CUPID), systems for PD that include the detection and intervention of FOG episodes (Cabestany et al., 2013; Mazilu et al., 2014, 2015, 2016), propose devices such as GaitAssist, and are equipped with two IMUs and a smartphone for active support of gait initiation, turns, and response inhibition, were developed (Mazilu et al., 2014).

\section{Recommendations and trends}

As with the other applications, the number of patients involved in these studies is not very high. Furthermore, not all the patients experienced FOG during experimental phases, so the datasets are further reduced. To avoid this issue, the systems also could be tested at home, at convenient times during the day, to capture a higher number of FOG events. However, the implementation of a long experimental protocol that includes cognitive and physical dual-tasks seemed to be able to trigger FOG episodes also in the laboratory setting (Capecci et al., 2016). Nevertheless the major difficulty for FOG detection is its unpredictability, so it would be preferable to test the wearable sensors in everyday situation and not while performing structured test (i.e., TUG test). Moreover, the large variability between clinicians suggests that caution should be used when comparing subjective ratings across centers (Morris et al., 2012). The majority of the works implemented experimental protocols that included all the part of freezing (i.e., starting, turning and narrow) while only some works (Handojoseno et al., 2012, 2013, 2014, 2015; Morris et al., 2012, 2013; Zach et al., 2015; Capecci et al., 2016) applied
TUG test for FOG detection, which is a test that not include "narrow" tasks. Generally the number and the duration of the freezing events were assessed, analyzing the gait of the patient directly or through a video recorded, but the reliability results of clinical assessment for these features were moderate (Morris et al., 2012; Tripoliti et al., 2013). Percent time frozen seemed to be a reliable metric of severity for both clinical and objective measures (Morris et al., 2012). In addition, the measurement of entropy allowed a detection of FOG events. Furthermore, this method is independent from the type of movement of the patient and the condition of the experiment; it is not based on thresholds and permits the detection of FOG events within longer periods of time while the patient performs daily activities (Tripoliti et al., 2013). Alternatively, the implementation of wavelet transform can have the advantage of providing localization in time and spectral domains, which is important for localizing the FOG events. Furthermore, because the use of a video recording to assess FOG is not always possible, different solutions should be found. The study of Morris et al. (2013) that employed computer-generated animation reconstructed by IMU data could be a promising solution, allowing for monitoring outside of the clinical environment, despite the complexity of this approach and the fact that motion artifacts in the computer-generated representation can affect event detection. Finally, at present, the use of videos seems to be mandatory to obtain good sensitivity and specificity, by establishing a FI (Moore et al., 2008) and thresholds to detect FOG events, because the use of IMU sensors only seems to identify several false positive detections (Zach et al., 2015) that should be avoided. The system for FOG detection should be user-specific (Capecci et al., 2016) and in real time, implementing algorithms able to effectively reduce the delay tolerance between FOG event detection and system reaction to promote a timely intervention (Bächlin et al., 2009) that could help the PwPD to avoid FOG episodes. Moreover, feedback should be context-aware, because continuous cueing is not appreciated by patients (Bächlin et al., 2010) and the efficacy of cueing could decrease over time. An interesting challenge would be freezing prediction instead of freezing detection (Mazilu et al., 2015), whereas possible integration of IMUs with other sensors to measure physiological parameters could provide a more complete analysis of patients' status related to the detection and prevention of FOG episodes, even if current results have limited accuracy (Handojoseno et al., 2012, 2013, 2014, 2015; Mazilu et al., 2015). Finally, the system should be usable outdoors, during unconstrained and unscripted activities (Cole et al., 2011), and be highly compact (Lorenzi et al., 2015), unobtrusive, light weight, easy to use, and meet the requirements of acceptability (Tripoliti et al., 2013; Capecci et al., 2016). In this direction, a smartphone-based system (Capecci et al., 2016) could be a valid solution that could allow patients to use the system during everyday activities and in the community, without discomfort. Also, the solution proposed by Mazilu et al. (2016), which looks for wrist sensors that can be included easily in a smartwatch or wristband, could represent a valid solution in terms of onbody acceptance and accessible technology, despite the fact that it comes at the cost of an increased number of false positives and a slight increase in detection latency. 


\section{Postural Instability}

Postural instability is one of the four cardinal motor symptoms in $\mathrm{PD}$, resulting in 14 papers in this review. The pull test is the main clinical examination to assess postural instability, as suggested by MDS-UPDRS, even if the equilibrium score derived from the Sensory Organization Test (SOT) is largely used. Postural instability accounts for $70 \%$ of PwPD that fall at least once each year, resulting in an increase of hospitalization and decrease in QoL (Ozinga et al., 2017). Postural control problems cause impairments in PD patients from the early stages of the disease (Gago et al., 2015; Masu et al., 2016). Furthermore, in pharmacologically untreated subjects (Mancini et al., 2011), prediction of postural instability enables prediction of future problems (Palmerini et al., 2011) and slows disease progression (Hubble et al., 2016) (Table 5). Sensor-based systems can improve PD diagnosis both in early disease, by measuring parameters not accurately identified by traditional tests (Hubble et al., 2016), as well as by distinguishing between mild and moderate disease stages (Masu et al., 2016). Pasluosta et al. (2015) proposed a new methodology to estimate postural sway to obtain unbiased and automated score prediction comparable to that rated by the physician. Gago et al. (2015), instead, tried to characterize the postural stability response to normal stance, Romberg test, and Ldopa treatment. PD patients showed high AP postural sway, increased jerk, and low responsiveness to Ldopa, correlated to their gait disturbance, resulting in the ability to identify PD in subjects at the early stage. Contrarily, jerk was slightly close to statistical significance in Hill et al. (2016). In a recent work, Baston et al. (2016) proposed the use of IMUs to quantify postural strategy and sway dispersion among $\mathrm{HC}$ and PwPD at different disease stages. Postural strategy was not affected by disease stage, but it was significantly lower in $\mathrm{ON}$ compared to OFF medication, and it was associated with selfperception of balance, while sway dispersion was significantly larger in the more severe $\mathrm{PD}$ group compared to the mild. In contrast, Mancini et al. (2016) focused on the APA prior to gait initiation and the first step, a state that is not readly observable to the naked eye. The system resulted in high test-retest reliability, both for $\mathrm{HC}$ and patients, and data measured from IMUs were highly correlated with those derived by validated systems. The peak of the ML acceleration during APA resulted in the most sensitive measure to the disease, with an amplitude significantly smaller in PD OFF compared to controls. Alternatively, Mellone et al. (2011) determined that since the tremor which is very common in PwPD can affect the identification of postural sway, appropriate techniques of filtering had to be adopted to remove tremor and preserve local dynamics without sacrificing frequency bandwidth. Additionally, the instrumented balance test can be adopted to classify PwPD on the basis of motor subtypes (i.e., dominant tremor or PIGD) (Rocchi et al., 2014) with high accuracy regarding clinical scales. Different motor subtypes show differences in biological and pathophysiological aspects, so their identification can be useful in large clinical studies and to promote accurate personalized therapies. Yelshyna et al. (2016) explored the mechanism underlying compensatory postural adjustments (CPA) by implementing a kinematic and time-frequency analysis based on IMU data during a virtual reality scenario to find differences between PwPD ON, PwPD OFF, and HC, to evaluate Ldopa effects. The lower band (LB) reflected the effect of Ldopa, while the higher band (HB) was responsible for the reaction to visual input-changing scenario; PwPD OFF showed abnormal CPA with respect to PwPD $\mathrm{ON}$ and HC in both bands. Finally, Hill et al. (2016) were the first to investigate the relationship of vision and visualcognition with postural control in PwPD compared to HC. Contrast sensitivity, visuo-constructive ability, and visuo-spatial ability were associated with postural control impairments in PD compared to age-matched HC. Visual biofeedback is important to maintain equilibrium, stability, and vertical body orientation, contributing also to a significantly decreased percentage of falls in PwPD in response to clinical pull test (Caudron et al., 2014).

\section{Recommendations and trends}

Even if the retropulsion test is included in the MDS-UPDRS 3.12 item to evaluate the postural stability, only two studies included in this review attempted to replace this test (Caudron et al., 2014; Hubble et al., 2016), probably due to methodological difficulties. The accelerometer-based approach makes it easier to quantify postural impairments compared to the conventional protocol with force plates, which are more expensive and not portable (Palmerini et al., 2011; Mancini et al., 2016; Ozinga et al., 2017). APA and postural sway, both in ML and AP directions, are the most analyzed features, able to differentiate between PD patients and HC (Ozinga et al., 2017) in the early stages of the disease (e.g., APA disruption can precede the compromission of the step execution) (Gago et al., 2015; Mancini et al., 2016), as well as between mild and severe PD groups (Baston et al., 2016) for differential diagnosis or between ON/OFF states for the advanced patients (Baston et al., 2016; Yelshyna et al., 2016). Alternatively, only one work took into account atypical parkinsonism (i.e., vascular PwPD) other than idiopathic PD patients (Gago et al., 2015), analyzing the response to L-dopa therapy. Additional sensory and attentional demands included in the experimental protocol can be helpful to identify the optimal features for disclosing postural differences between PD and $\mathrm{HC}$ subjects (Palmerini et al., 2011).

\section{Upper Limbs}

According to literature, several groups have studied the use of wearable sensors for the analysis of upper limb motion (15 papers were included). In particular, two main approaches have been followed: the use of simple sensors (e.g., on fingers or wrists) or the integration of the sensors in a sort of glove (e.g., $\mathrm{TG}^{\circledR}$ medical glove and MiMed smart glove) (Table 6). An alternative solution was proposed by Cavallo et al. (2013) who developed a wireless wearable modular device called SensHand equipped with IMUs placed on the wrist and on the distal phalanx of thumb, index, and middle fingers. The device was not a traditional glove in terms of wearability and modularity, but an integration of inertial sensors. The use of a combination of inertial sensors adequately placed on arms, forearms, hands, and finger segments allowed the measurement of a wide range of parameters both in spatiotemporal and frequency domains. EMG signals, only, were analyzed by Robles-Garcia et al. to 
assess the variability of the movements in finger tapping (RoblesGarcía et al., 2013). In most of the works, the examiners asked to the patients to perform standard task items described in MDSUPDRS III (e.g., alternating hand movements, finger-to-nose, finger tapping) for upper limb motion analysis (Fukawa et al., 2007; Okuno et al., 2007; Patel et al., 2009; Yokoe et al., 2009; Hoffman and McNames, 2011; Cavallo et al., 2013; Robles-García et al., 2013; Jia et al., 2014; Delrobaei et al., 2016; Djurić-Jovičić et al., 2016; Eskofier et al., 2016). The papers looked for the correlation between the features extracted and the clinical scores assigned by clinicians on the UPDRS (Okuno et al., 2007) using Pearson (Robles-García et al., 2013) or Spearman correlation (Delrobaei et al., 2016; Djurić-Jovičić et al., 2016), multiple linear regression (Cavallo et al., 2013), ANN (Fukawa et al., 2007), quadratic, and nearest mean scaled classifiers (DjurićJovičić et al., 2014a), and they presented a good data separation in clusters between different groups of subjects (Cavallo et al., 2013; Djurić-Jovičić et al., 2014a; Delrobaei et al., 2016). For example, Cavallo et al. (2013) showed better performance in terms of frequency, velocity, and amplitude of movement for HC compared to PwPD, whereas Patel et al. (2009) provided analysis of bradykinesia and dyskinesia with low estimation error values. Also, Eskofier et al. (2016) performed an assessment of bradykinesia, but they introduced the use of deep learning instead of machine learning techniques as a promising method to analyse wearable sensor data. Bradykinesia also was objectively assessed by Delrobaei et al. (2016), who provided for a new index (i.e., BKI score) to express a quantification of this symptoms in upper limbs, according to UPDRS. Djurić-Jovičić et al. (2014a) defined areas and volumes related to tapping activities that can be used to quantify the movement vigor and highlight their decrement over time, which is typical for PwPD caused also by fatigue (DjurićJovičić et al., 2016). Yokoe et al. (2009) proposed the opening velocity in the finger-tapping task as a novel parameter for the discrimination of PD patients, whereas Okuno et al. (2007) focused on contact force in finger tapping to predict the level of the pathology. A different approach was proposed by Barth et al. (2012), who used a Biometric Smart Pen (BiSP) equipped with a triaxial accelerometer, finger grip force during holding the pen, refill force, and vibration sound, able to measure handwriting, drawing, and gesture movements on paper or in free air.

\section{Recommendations and trends}

The clear trend in terms of wearable technology is the development of wireless, unobtrusive, quiet, and washable devices that are easy to use without a technician's support (Cavallo et al., 2013). Triaxial inertial sensors seem to be preferable rather than uniaxial accelerometers (Patel et al., 2009) because the former provides the possibility to analyse the motion not only in a plane (e.g., AP plane, sagittal plane) but in a complete $3 \mathrm{D}$ space. In addition, combining results obtained by sensor pairs to characterize motion patterns that correspond to normal activity and detect their transition into abnormal ones is easier using triaxial inertial sensors. Further, to acquire data able to finely measure the motion of the hand and fingers, a high sampling frequency of the sensors is needed (e.g., $100 \mathrm{~Hz}$ ) (Patel et al., 2009; Hoffman and McNames, 2011; Cavallo et al.,
2013), instead of low acquisition rate (Jia et al., 2014). Features that are extracted with Continuous Wavelet Transform (CWT) (Djurić-Jovičić et al., 2014a) or entropy (Patel et al., 2009), even if they can discriminate well between PwPD and HC, can be difficult to interpret for clinical staff. Biomechanical measures such as velocity, frequency, or displacement of movements (Yokoe et al., 2009; Cavallo et al., 2013; Delrobaei et al., 2016) provide results that are more appropriate, easier to understand, and more similar to the assessment required by MDS-UPDRS. Halts and hesitations are important parameters, as well, to evaluate the severity of the diseases for tasks such as finger tapping, but no study has proposed their exact calculation based on inertial signals. The calculation of indexes able to quantify the severity of PD symptoms, such as the BKI score for bradykinesia assessment (Delrobaei et al., 2016), are encouraging, because the development of such indicators could overcome the issues of subjectivity and inter-rater variability that currently afflict the diagnosis of the pathology. Furthermore, the implementation of these indexes could assist with home monitoring and personalized assessment and treatment of the symptoms. Further, some features can have a potential use to achieve optimal stimulator settings for DBS, a technique widely used in PwPD, especially in advanced stages of the disease to improve and slow the symptoms of the pathology. Even if DBS effects result in high inter-subject variability, and different DBS settings show high intra-subject variability, the EMG features proposed by Rissanen et al. (2015) could detect motor symptoms that kinematic measurements or clinical evaluation cannot detect, and they can help the clinicians in arriving at optimal DBS settings more quantitatively. To obtain predictive values from motion analysis, Hoffman and McNames (Hoffman and McNames, 2011) proposed a comparison between different adaptive filtering algorithms: least mean square and Kalman filter show the best results in predicting angular velocities and angular values of the movements performed by the patients.

\section{Other Symptoms: Leg agility, Rigidity, and Arms Swing}

A task specifically requested by the UDPRS scale for motor evaluation of lower limbs is the leg agility task (LA). It consists of raising the foot from the floor as fast as possible, starting from a sitting posture, for 10 repetitions. A wide number of features can be measured by placing an inertial sensor on each thigh and analyzing this exercise (Giuberti et al., 2014; Parisi et al., 2015), because thigh inclination and heel elevation are highly correlated, as demonstrated by comparing results from the sensors with those of an optoelectronic system. A good correlation emerged between the extracted features and the score assigned by expert neurologists on the UPDRS scale. The use of a wireless body sensor network (BSN) makes the proposed system directly integrable into IoT systems.

Rigidity is one of the four cardinal symptoms in $\mathrm{PD}$, but uncertainty exists about the best method to evaluate it. Rissanen et al. (2009) proposed to measure the dynamic muscle contraction and distinguish between PD patients and HC by analyzing EMG and acceleration signals acquired during elbow flexion and extension movements. The results showed that 
these dynamic measurements can be informative for assessing neuromuscular dysfunction in PD, even if the accuracy in assessing the patients and the controls was not very high, especially considering that Parkinsonian subjects had low scores in the UPDRS scale for rigidity, finger tapping, and tremor tasks (Table 7). According to the MDS-UPDRS item 3.3, the rigidity should be assessed during passive movements, so methodological difficulties can occur in measuring the rigidity response of PD patients using wearable sensors. This could be responsible for the very limited number of papers found about the measurement of this impairment.

The assessment of the arm swing using ultrasound-based motion analysis during treadmill walking is useful to identify PwPD, particularly when they are subjected to dual tasks, as well as in response to adaptation of the pharmacological treatment. Specifically, asymmetry indexes based on angular amplitude of the movement calculated for both of the arms were analyzed and compared by Sant'Anna et al. (2011), resulting in good discrimination between PwPD and HC in the first stage of the disease.

\section{Application 4: Motor Fluctuations and On/Off Phases}

Levodopa (Ldopa) is the most common pharmacological therapy adopted by PwPD. It is a treatment able to partially reactivate the neural connections that control the temporal patterns responsible for performing the activity, because Ldopa is converted to dopamine, which is lacking with the progression of the pathology. Also, several side-effects are caused by Ldopa therapy, particularly dyskinesias (referred to as Ldopainduced dyskinesias or LID), and discrimination between these movements and voluntary motion is difficult to achieve using wearable sensors. PD patients in mid-stage and advanced disease often suffer from motor-fluctuations which represent a severe motor disorder that negatively influences health-related QoL in those patients. Several papers exist in literature about the use of devices based on inertial sensors to monitor the motor fluctuations that affect PwPD, especially in the late stage of the pathology and to evaluate therapy response (12 papers were included in the review). Accelerometers and gyroscope MEMS were placed on different body segments (e.g., wrist, upper arm, thigh, shin, foot, trunk) and combined with other sensors (e.g., EMG or ECG) as reported in Table 8. To monitor the fluctuations that can appear during the day in a patient, both linear and nonlinear features were analyzed. Patel et al. (2009) proposed for monitoring of motor fluctuations; dyskinesia and bradykinesia assessment; and measurements of the intensity of acceleration, modulation, rate, regularity, coordination between right and left sides, and entropy in a continuous monitoring. Cancela et al. (2011), instead, focused on spectral analysis, showing that the power spectrum in PwPD is wider than in HC, and the power in the main peak moved to different frequency bands, generating new peaks with a significant power. Samà et al. (2012) also focused on the spectral analysis of the accelerometer signal, defining frequency thresholds able to identify if a patient suffers from dyskinesia, avoiding false positive detections, similarly studied by Pérez-López et al. (2016). Further, they provide for an ON/OFF state algorithm detection, based on stride characterization during walking, since OFF states results in lower amplitude in both temporal and frequency domain. To evaluate the pharmacological therapy taken by the patients, instead, Ruonala et al. measured ECG-derived parameters both in time and frequency domain, demonstrating that some parameters effectively decrease in a significant way, between off and on medication (Ruonala et al., 2015). Hssayeni et al. (2016) focused on signal power, jerk, entropy, peak-to-peak, and correlation coefficient and developed a semi-supervised clustering approach, $\mathrm{k}$-means based, to automatically assess the ON/OFF medication states of PwPD. Finally, Ramsperger et al. (2016) measured dyskinesia as the ratio of the angular rate around the $\mathrm{z}$-axis over the angular rates lying within the xy-plane, as measured by the triaxial gyroscope sensor within the SENSE-PARK European research project. The previous features were extracted following specific experimental protocols that included standard diagnostic exercises according to the UPDRS scale such as finger-tonose, tapping, sit-to-stand, walking, stand-to-sit, finger tapping, alternating hands movements, heel tapping (Patel et al., 2009; Rissanen et al., 2011); or allowing subjects to do everyday free activities (e.g., walking, reading, eating) (Ramsperger et al., 2016); or prescribing specific daily activities (e.g., cutting food, dressing) Samà et al. (2012) (Hssayeni et al., 2016); or permitting a combination of both typos of movements (Pastorino et al., 2011; Rahimi et al., 2011; Tsipouras et al., 2012) eventually with some restrictions (e.g., subject seated) (Tsipouras et al., 2011). The aim of these studies was to identify different motor states (Patel et al., 2009; Samà et al., 2012; Hssayeni et al., 2016); quantify the efficacy of treatment (Ruonala et al., 2015) and DBS (Rissanen et al., 2011) in PD to assess the severity of bradykinesia (Pastorino et al., 2011), dyskinesias (Pérez-López et al., 2016; Ramsperger et al., 2016), and LID (Tsipouras et al., 2011); and to manage them (Tsipouras et al., 2012). For this reason, permitting the use of the system at home in an unsupervised environment, at times, is important (Pastorino et al., 2011; Ramsperger et al., 2016).

\section{Recommendations and Trends}

The study and identification of motor fluctuations in PwPD is a challenge in the long-term management of the pathology. The OFF states can appear during the day, when the effect of the drugs consumed by patients is reduced and the severity of the symptoms comes out or re-emerges. When a patient is in the OFF phase, the patient's condition can be considered critical, and the patient can be subject to the risk of falls, FOG events, LID, significant tremor, and general difficulties in performing daily activities. Commonly, this situation is not manifested when a patient is under medical examination in a hospital or in ambulatory monitoring, so the ability to control the subject at home throughout the day is essential to identify and to prevent, if possible, these critical events. The pharmacological treatment most commonly used for PD is based on Ldopa. This drug effectively holds off the motor symptoms of the pathology (Ruonala et al., 2015), but at the same time, it can be responsible for side effects such as LID that, in turn, can be very disabling for PwPD and predictive for risk of falls (Ramsperger et al., 
2016). An adequate monitoring of LID is needed to adjust the pharmacological treatment followed by the patients and ensure the benefits derived from an optimal drug therapy (Pérez-López et al., 2016). However, the detection of LID severity, particularly for slightly impaired patients, is not easy to obtain (Tsipouras et al., 2011). Because of the lack of objective methods for quantifying the efficacy of treatment in PD, new strategies should be implemented (Rissanen et al., 2011; Ramsperger et al., 2016). Since the border between ON and OFF depends on the stage of the disease and on the patient, thresholds that best distinguish both motor states in a certain patient are not expected to be the best thresholds for another patient (Samà et al., 2012). The inter-individual differences between patients, in fact, can lead to different responses both on therapy treatment and motor states. To date, the best results in identifying different motor states are restricted to patients with a great improvement in tremor and bradykinesia from medication OFF to ON stage (Hssayeni et al., 2016). Long-term experimentations in home settings appear necessary to obtain valuable data that allow an accurate assessment of ON/OFF motor states in PwPD and dyskinesias (Ramsperger et al., 2016).

\section{Application 5: Home and Long-Term Monitoring}

To date, as clinical scales are the gold standard for in-clinical setting assessment of PD, the use of patient-completed symptom diaries is the current gold standard for the home monitoring of the pathology (Fisher et al., 2016). Recent studies proposed the use of commercial devices such as the Microsoft Kinect sensor (Paredes et al., 2015) as a low-cost solution to assess the movement of Parkinsonian patients, not only in clinical settings, but also at home. Nevertheless, the accuracy of these systems can be considered good in the measurement of spatiotemporal features for gross movements, but it is not acceptable compared to validated motion capture systems, which are the gold standard for fine movement analysis of actions such as hand clasping or finger tapping, which is required in the MDS-UPDRS scale for PD severity evaluation.

The concept of monitoring patients in their own homes is the future trend in terms of long-term monitoring, instead of the typical ambulatory monitoring proposed in previous works (Salarian et al., 2007a), even if with good results. Recently, the European Committee has emphasized this direction, as demonstrated by the grant of different projects for home monitoring. PERFORM, for example, consisted of design, development, validation, and exploitation of a multi-parametric system for the long-term, continuous, and effective assessment and monitoring of motor status in PD using the PERFORM WMSMU, a wearable multi-sensor monitoring unit (Tsipouras et al., 2014). The system aimed to allow the physicians to monitor and detect changes in the symptomatic behavior as quickly as the changes appear (Cancela et al., 2010). Also, the European research project $\mathrm{CuPiD}$ looked for home environment applications. In particular, within this project, a system composed of IMUs and smartphone-based application was developed with the aim to provide an efficient gait training application at home for PwPD (Ginis et al., 2016). They system gave real-time measurement of gait, auditory biofeedback on spatiotemporal gait parameters, and rhythmical auditory cueing to prevent or overcome FOG episodes. The system was able to improve gait and balance in PwPD in a more effective way than traditional homebased gait intervention as well as follow-up controls. Moreover, it appeared well-tolerated and user-friendly for PwPD, even for those who were unfamiliar with a smartphone. The systems proposed in literature (14 papers included in this review) for monitoring and managing the development of the disease at home are equipped with triaxial accelerometers and gyroscopes, MEMS or EMG devices, placed on different body segments (e.g., wrist, ankle, waist, thigh, shin) for a full wearable system that did not impose limitations to patients' movements (Table 9). The data logger was required to be portable and multifunctional, and a robust control on the device was adopted to inform the patient about the treatment program or to allow the sending of emergency calls if needed (Tsipouras et al., 2014). Alternatively, Cook et al. (2015) proposed the CASAS Smart Home, in which sensor data from ambient sensors are added to wearable sensors and smartphone. The system included a large number of sensors, but it allowed extraction of a considerable number of features, revealing differences between tasks performed by HC and PwPD and providing an automatic classification of end users between $\mathrm{HC}$, subjects with mild cognitive impairment (MCI), and PwPD with or without MCI. To control the development of the disease, different approaches are adopted, as reported in literature. In some works the patients are asked to perform standardized motor tasks only, as those described in the motor section of MDS-UPDRS (e.g., quiet sitting, finger tapping, finger-to-nose, alternating hand movements, heel tapping, walking) (Patel et al., 2009; Jaywant et al., 2016). Other works required instead the performance of ADL or similar common everyday tasks (i.e., preparing snacks, eating, reading, writing, using of Internet, conversing with someone) (Cancela et al., 2010; Rahimi et al., 2011; Khan et al., 2014; Lambrecht et al., 2014; Cook et al., 2015; Fisher et al., 2016), whereas further works aimed to continuously monitor the patients (Pastorino et al., 2011; Roland et al., 2013, 2014; Wallén et al., 2014), eventually even during the night (Fisher et al., 2016). In terms of measurements, a wide range of features were extracted for long-term monitoring at home, including statistical and frequency features, gait parameters, Fourier coefficients, and many others.

The system proposed by Fisher et al. (2016) was composed of wrist-worn accelerometers to acquire data in laboratory and home environment and ANN to compare the sensor data with the diaries of patients as self-reported. Diaries and clinicianrated assessments were compared. High specificity both in laboratory and at home was seen for dyskinesia, but with low sensitivity at home, perhaps because the system is based on wrist-worn accelerometers. Thus, dyskinesia that can occur in other body segments cannot be assessed. High correlation was found between sensor data and diaries regarding the amounts of time in a given disease state. However, since the ideal PD home monitoring system should be real time and adaptable, and in light of the information obtained, the real-time evaluation with the proposed sensor system is not feasible. A different analysis was 
conducted by the research group of Roland et al., who pointed out the differences between PwPD and HC, but also between genders on the basis of features extracted by EMG signals, and found significant dissimilarities (Roland et al., 2013, 2014). Finally, Jaywant et al. (2016) studied the efficacy of home-based gait observation training to enhance walking in PD. Objective changes in gait did not result, but an increased self-perceived mobility was reported. Since this aspect is evaluated through the administration of PDQ-39 mobility subscale, it is possible that accelerometers did not measure the kinds of functional improvements perceived by the patients and expressed in the clinical scale. Furthermore, the system was feasible and easy to understand and to use.

\section{Recommendations and Trends}

The principal aim of the home monitoring is to provide an optimal management of PD. According to literature results, this can be done by observing the development of the pathology through the analysis of data acquired by wearable sensors, which seem to be the best type of devices to adopt. The implementation of a Smart Home, in fact, lowered the users' acceptance of the technology and resulted in an invasive system that did not provide a sufficiently high accuracy in observations and also measured numerous irrelevant features (Cook et al., 2015).

To obtain a useful monitoring system, the clinicians should be able to interact remotely with patients in the home setting, to configure the sensor nodes for the application at hand, and to record annotated data with the possibility of video conferencing, real-time data visualization, data collection supervision, annotation tools, and spot checks. Collecting data in the home setting and remotely monitoring the patient would allow clinicians to improve quality of care of the patients while reducing the costs. From a technical point of view, the system should be able to work in an unsupervised environment (Pastorino et al., 2011; Roland et al., 2013, 2014; Wallén et al., 2014; Fisher et al., 2016), provide real-time biofeedback (Ginis et al., 2016), feature user-friendly interfaces both for clinicians and patients, and attend to the real-time transmission of the data acquired to prevent data loss caused, for example, by absence of available Internet service, to be acceptable and usable (Fisher et al., 2016; Ginis et al., 2016; Jaywant et al., 2016).

At the moment, the large variability resulting within subjects for each task, across tasks for individual subjects, and between scripted and unscripted tasks is a crucial point to overcome to ensure the correct assessment of the status of the patient. The use of a large number of sensors seems, for instance, the most feasible solution to capture the wide range of movement patterns adopted by PwPD to perform required tasks (Rahimi et al., 2011). On the contrary, to address acceptability and usability requirements, a reduced number of sensors is more appropriate, as with the wrist-worn devices proposed by Fisher et al. (2016), which are usable also in the home environment and do not compromise the execution of common ADL. Moreover, large dimension and weight of wearable devices should be avoided because they are not comfortable (Pastorino et al., 2011).

For future works, the system should be totally automatic and avoid the requirement that patients must introduce information manually about medication or meal intake using the developed GUI, or compile a daily diary (e.g., every half-hour). Finally, to obtain real-time assessment of the patients' status, different machine learning methods were, and the SVM with Gaussian radial kernel seemed to be the best classifier for detecting PD (Khan et al., 2014).

\section{DISCUSSION AND CONCLUSIONS}

PD is a disabling pathology that affects millions of people worldwide. Since the disease heavily influences the QoL of patients, raising the burden of care on their relatives and the costs for health and care for the society, an optimal solution for the management and treatment of PD is needed. This paper focuses on the use of wearable devices for PD applications (early diagnosis, tremor, body motion analysis, motor fluctuations and ON-OFF phases, and home and long-term monitoring), to analyse the current state-of-the-art of existing systems used in this field and to identify the pros and cons for each work with the aim to give recommendations for future improvements.

Currently, PD is diagnosed when wide areas of the brain are already damaged, because the patients go to the clinician when motor symptoms are evident and begin to influence their common activities. Thus, the diagnosis is generally made when the brain neurodegenerative process is already triggered, whereas to improve the treatment of the pathology, PD should be diagnosed when it is at the onset. Recent trends show that it is very important to identify the disease in the early stage, possibly in the prodromal phase, when the symptoms are not yet evident, to optimize the management of the pathology and to improve the quality of care and consequently the QoL for the patients. Currently, the neurologist diagnoses the pathology by asking the patient to perform tasks defined in the MDSUPDRS (Goetz et al., 2008) and assessing the patient through a visual examination. The scale adopted is semi-quantitative, and the neurologist assigns a score between 0 (normal state) and 4 (severe stage of PD) for each task performed and then sums the scores for all the items. This type of evaluation is subjective, based on the experience of the clinician, and generally it can vary between different neurologists and health centers, making the diagnosis inaccurate or uncertain, at times. Additionally, to confirm the diagnosis, invasive and expensive nuclear medicine tomographic imaging techniques are generally adopted (i.e., single proton emission computed tomography-SPECT-with DaTscan) with high costs for healthcare. For these reasons, in recent years, different research groups have worked to find a method to objectively measure the motor performance of the patients, since the motor symptoms are those that generally lead the neurologist to the diagnosis. The idea is to quantify the motor skills of the patients, finding a way to measure the items proposed in MDS-UPDRS III. A method to objectify the motion can lead to a quantitative diagnosis of the $\mathrm{PD}$, overtaking the problems linked to the subjectivity and to the inter-rater and intra-rater variability, thus increasing the accuracy of the diagnosis. A study (Ghassemi et al., 2016) revealed that the overall classification rate is not only limited by technical accuracy, 
but also by clinical accuracy. In this direction, different works proposed well-defined experimental protocol to replicate the MDS-UPDRS III items, looking for a close correlation between the parameters measured with the technological solution adopted and the clinical scores assigned by the neurologist. System cost and portability are two important characteristics that must be considered when developing a novel diagnostic tool. To measure the motor performance of the patients, the most appropriate way seems the use of wearable devices based on inertial sensors, which can acquire data with a high sample rate (e.g., $100 \mathrm{~Hz}$, Mellone et al., 2011; Palmerini et al., 2011; Cavallo et al., 2013; Sejdić et al., 2016), and examination of the results on board or transmittal of results to a control station for offline data processing. Thanks to the recent advances in MEMS technology, this type of device is portable, light weight, unobtrusive, easy to use, inexpensive, and accurate in the measurements. Thus, wearable devices with inertial sensors can represent an optimal solution in healthcare applications, for use in both clinical infrastructure and the home environment. Similarly, traditional motion capture tools such as multi-camera retro-reflective motion analysis systems, while potentially effective at finely measuring the motor performance of the subjects, are nonetheless difficult to bring out of a laboratory setting because of their cost and non-portability. Although features extracted from 3-D motion data are slightly more accurate than features extracted from inertial sensor data, inertial sensors are non-invasive and can be used continuously and in uncontrolled environments (Sant'Anna et al., 2011).

The possibility to use the system not only in controlled health infrastructures, but also in unsupervised environments (homebound setting, over a 24-h cycle) (Pastorino et al., 2011; Fisher et al., 2016; Ramsperger et al., 2016) and during unscripted tasks (Cole et al., 2011; Roy et al., 2011) is crucial to improve the monitoring and assistance for PD patients during common everyday activities. For this reason, the devices must be tested fully, not only for the typical items proposed in MDS-UPDRS III, which are needed for the diagnosis, but also for the ADL and other tasks of everyday life (Salarian et al., 2007b; Bächlin et al., 2009, 2010; Mazilu and Hardegger, 2012; Lambrecht et al., 2014; Cook et al., 2015; Fisher et al., 2016; Rigas et al., 2016). Since video recording for long-term monitoring during free daily activities is not possible (Salarian et al., 2007b), the use of wearable sensors is the most promising solution for this purpose, with the plan to eventually implement them into IoT systems (Giuberti et al., 2014). An alternative approach in order to avoid high variability in remote monitoring could be the use of Virtual Reality technologies. They, in fact, could be an useful tool for an homogeneous approach of monitoring patients at home and they could be suitable also for other applications (i.e., rehabilitation) in PD (Dockx et al., 2016).

Moreover, to guarantee an optimal management during the home monitoring, it is mandatory to develop and provide userfriendly interfaces that allow the clinicians and the patients to stay in contact (Pierleoni et al., 2014), adopting a sort of telemedicine service that permits exchanging of information, consulting service, and therapy adjustments. At the beginning, patients, caregivers and medical staff should be trained to use this technology, adopting educational strategies that could increase the level of confidence with the proposed solutions. Then, with automatic updates to a mHealth server, caregivers and healthcare professionals can gain insights into overall wellness of the subjects by analyzing the parameters from multiple tests performed in a single day or monitoring and evaluating the evolution of disease by analyzing the trends in the parameters collected over longer periods of time. In this direction, the recent EU PD_Manager Project (Rigas et al., 2016) was targeted the development of a complete mHealth PD management solution through Microsoft band and Android application. The real-time (Ferrari et al., 2016) and automated assessment of the performance of the patients (Giuberti et al., 2015) is another cardinal point for the development of an efficacious remote support and monitoring system. It allows assessment of the status of the patients on demand, evaluating also the eventual changes due to modification in pharmacological or rehabilitative treatment, as well as objective evaluation of the efficacy of the adopted therapy. In fact, it is widely recognized that self-report assessments can be limited by patients over- or under-reporting their difficulties. Hence, more objective tests would greatly benefit the clinical assessment of PwPD (Hubble et al., 2016). Finally, an adequate monitoring and assistance system at home could reduce the number of medical examinations in hospital, promoting the empowering and the self-consciousness of the PwPD in the management of their own disease, and stimulating them to improve the performance in following a personalized therapeutic pathway in developing specific models for home monitoring.

As reported in Table 10, a significant limitation of most studies reported in literature is the small dataset adopted to test the technological solutions proposed (Figure 2D). The small sample size reduces the generalizability of the results, which should be verified in a larger sample (Mariani et al., 2013; Palmerini et al., 2013) and, specifically, longitudinal and large sized validation is needed to prove clinical applicability (Ghassemi et al., 2016) of the developed technologies.

Another open issue in the analyzed works concerns the optimal number of sensors to use for recording patient activities. The literature has a lack of consensus regarding the optimal number of sensors, and the optimal site for their placement, for the assessment of PD motor symptoms (Fisher et al., 2016). The sensors should guarantee that the subjects can perform the movements without restrictions. If a reduction in the number of sensors may lead to loss of potentially relevant information (Pastorino et al., 2011), to avoid a high invasiveness of the system, a restricted number of sensors must be used (Salarian et al., 2013), because using up to three on-body sensors together with a phone decreases the acceptance of the system (Mazilu et al., 2016). Thus, a trade-off must be found and adopted. The acceptability of the system would be considered, particularly for smart home monitoring and long-term assessment of motor symptoms (Barth et al., 2011), when a prolonged use of the devices is required. In this direction, recent studies (Arora et al., 2014; Kostikis et al., 2015; Capecci et al., 2016; Ferrari et al., 2016) proposed the use of 
TABLE 10 | Limitations and opportunities.

\begin{tabular}{|c|c|c|}
\hline TYPE & Barriers/limitations & Challenges and opportunities \\
\hline \multirow[t]{3}{*}{ Experimental } & Dataset & High number of subjects must be recruited to validate the results of the studies. \\
\hline & Dataset & Patients with mild impairments $(\mathrm{HY}=1)$ should be investigated to promote early diagnosis. \\
\hline & Dataset & Age-matched controls should be involved, because motor performance vary with age. \\
\hline \multirow[t]{6}{*}{ Technological } & Acceptability & The system should be developed with consideration for patients' requirements and needs. \\
\hline & Usability & $\begin{array}{l}\text { The system should be easy to use and eventually provide user-friendly interfaces both for clinician and } \\
\text { patients. }\end{array}$ \\
\hline & Portability and unobtrusiveness & $\begin{array}{l}\text { The system should be wireless and non-invasive for use at home or outdoors without limitations for the } \\
\text { patients. The number of sensors used and their placement over the body should address the trade-off } \\
\text { between accuracy in measurements and obtrusiveness. }\end{array}$ \\
\hline & Long-term monitoring & $\begin{array}{l}\text { The system should ensure long-term operation. Long-term battery and low-power solutions have to be } \\
\text { adopted, especially for home-monitoring applications for advanced patients with motor fluctuations. }\end{array}$ \\
\hline & Measurements & $\begin{array}{l}\text { The system should provide quantitative, accurate, precise, objective, and reliable measurements of the } \\
\text { symptoms analyzed to actively support the clinician in diagnosis and management of the pathology. }\end{array}$ \\
\hline & & $\begin{array}{l}\text { The system should be able to recognize different stages of the disease as well as to evaluate changes due to } \\
\text { pharmacological treatments. }\end{array}$ \\
\hline Clinical & Availability for use in clinical practice & $\begin{array}{l}\text { - Accuracy, precision, reliability, and dependability of the system should be ensured. } \\
\text { - Objective support for clinical examination and assessment should be validated, complying with traditional } \\
\text { medical scales. } \\
\text { - Improvements for patients' management should be efficiently demonstrated. }\end{array}$ \\
\hline
\end{tabular}

smartphones that are equipped with inertial sensors. This device is a common technological tool that does not require specific technical capabilities for the use and is widely accepted and usable, not only in healthcare infrastructures, but especially at home, outdoors, and in the community.

Moreover, the features to extract from the inertial signals and to provide to the clinical staff are a matter of debate. Generally, they are selected by using statistical techniques such as ANOVA or the Mann-Whitney test, which can identify the features that best discriminate between different subjects' groups (i.e., patients and $\mathrm{HC}$ ). Even if this is mathematically correct, the features proposed are not always easy to understand by the clinicians. It is important, instead, to provide the clinical staff with a reduced and comprensive set of features to avoid misleading in clinical practice (Palmerini et al., 2011).

Finally, to provide automatic (almost real time) assessment of the performances measured, recent works implemented different machine learning approaches (Kostikis et al., 2015), such as SVM (Patel et al., 2009), NaiveBayes, kNN, NCC, RF (Reinfelder et al., 2015), decision tree (DT), and LDA (Barth et al., 2011; Perumal and Sankar, 2016). All of these classifiers were used to discriminate between PwPD and patients with similar symptoms but different pathologies (e.g., subjects with ET) (Surangsrirat et al., 2016) or to distinguish between PwPD and $\mathrm{HC}$, or to identify different stages of the disease, or to evaluate the medication and DBS effects (Rissanen et al., 2011). In various works, the Principal Component Analysis (PCA) is implemented as well, generally for the dataset reduction and feature selection (Palmerini et al., 2011; Parisi et al., 2015), or for visual inspection analysis. In fact, the reduceddimensional system of the principal components could be used for explorative analysis (e.g., for clustering subjects with the same behavior) and to detect outlier subjects, which have a performance very different from the average. This technique is often used in combination with the discussed machine learning techniques (Rissanen et al., 2009; Tien et al., 2010; Giuberti et al., 2015; Parisi et al., 2015; Ghassemi et al., 2016).

Alternatively, a recent work proposed the use of deep learning as a promising method to analyse wearable sensor data in place of machine learning approaches (Eskofier et al., 2016). Advantages of deep learning are (i) there is no need to rely on expertdefined features that may or may not represent the information content of the signal that is subjected to classification; (ii) the analysis procedure resembles what human experts do, since the whole signal segment is rated with one continuous and clinical scale-like output; (iii) an adaptation of the network to an individual patient is possible; (iv) deep learning based frameworks, in particular, can be expected to produce better results with the growing amount of data that will become available. However, to manage these data, high quality labels are required from clinical experts. Deep learning may provide higher accuracy than machine learning (e.g., AdaBoost.M1, RF, kNN, SVM) for correctly classifying symptoms and subjects, but further and extended studies are required to validate this theory.

In conclusion, this review provides a wide overview of the technological solutions currently implemented for PD applications. The rising idea from literature is to use unobtrusive and accurate systems that could monitor the disease progression since its beginning, throughout its development. Appropriate technological solutions, in fact, could improve the management of the PD, enhancing the QoL of PwPD, through an early 
and objective diagnosis of the pathology, as well as monitoring the effects of the pharmacological therapy during the disease progression.

\section{AUTHOR CONTRIBUTIONS}

ER was responsible for paper structure and writing, synthesizing the information from the papers into text and tables. CM was the clinical supervisor, responsible for clinical aspects and contributing in introduction, methodology definition and search strategies. FC was the scientific supervisor, guarantor for the review and contributed in methodology definition, paper writing, discussion and conclusion. All authors were involved in papers screening and selection. All authors read, provided feedback and approved the final manuscript.

\section{REFERENCES}

Alhamid, M. F., Alamri, A., and El Saddik, A. (2010). "Measuring hand-arm steadiness for post-stroke and Parkinson's disease patients using SIERRA framework," in International Workshop on Medical Measurements and Applications (MeMeA) (Ottawa, ON: IEEE), 6-9.

Al-Jawad, A., Adame, M. R., Romanovas, M., Hobert, M., Maetzler, W., Traechtler, M., et al. (2012). "Using multi-dimensional dynamic time warping for TUG test instrumentation with inertial sensors," in International Conference on Multisensor Fusion and Integration for Intelligent Systems (MFI) (Hamburg: IEEE), 212-228.

Arora, S., Venkataraman, V., Donohue, S., Biglan, K. M., Dorsey, E. R., and Little, M. A. (2014). "High accuracy discrimination of Parkinson's disease participants from healthy controls using smartphones," in International Conference on Acoustics, Speech and Signal Processing Proceedings (Florence: IEEE), 3641-3644.

Bächlin, M., Plotnik, M., Roggen, D., Inbar, N., Giladi, N., Hausdorff, J., et al. (2009). "Parkinson's disease patients' perspective on context aware wearable technology for auditive assistance," in $3 r d$ International Conference on Pervasive Computing Technologies for Healthcare (PervasiveHealth) (London: IEEE), $1-8$.

Bächlin, M., Plotnik, M., Roggen, D., Maidan, I., Hausdorff, J. M., Giladi, N., et al. (2010). Wearable assistant for Parkinson's disease patients with the Freezing of Gait symptom. IEEE Trans. Inf. Technol. Biomed. 14, 436-446. doi: 10.1109/TITB.2009.2036165

Barth, J., Klucken, J., Kugler, P., Kammerer, T., Steidl, R., Winkler, J., et al. (2011). "Biometric and mobile gait analysis for early diagnosis and therapy monitoring in Parkinson's Disease," in 33rd Annual International Conference of the IEEE Engineering in Medicine and Biology Society (EMBC) (Boston, MA: IEEE), 868-871.

Barth, J., Oberndorfer, C., Kugler, P., Schuldhaus, D., Winkler, J., Klucken, J., et al. (2013). "Subsequence Dynamic Time Warping as a method for robust step segmentation using gyroscope signals of daily life activities," in 35th Annual International Conference of the IEEE Engineering in Medicine and Biology Society (EMBC) (Osaka: IEEE), 6744-6747.

Barth, J., Sünkel, M., Winkler, J., Eskofier, B., and Klucken, J. (2012). “Combined analysis of hand and gait motor function in Parkinson's disease," in 34th Annual International Conference of the IEEE Engineering in Medicine and Biology Society (EMBC) (San Diego, CA: IEEE), 5122-5125.

Baston, C., Mancini, M., Rocchi, L., and Horak, F. (2016). Effects of Levodopa on Postural Strategies in Parkinson's disease. Gait Posture 46, 26-29. doi: 10.1016/j.gaitpost.2016.02.009

Bazgir, O., Frounchi, J., Habibi, S. A. H., Palma, L., and Pierleoni, P. (2015). “A neural network system for diagnosis and assessment of tremor in Parkinson disease patients," in 22nd Iran Conference on Biomedical Engineering (ICBME) (Iran: IEEE), 1-5.

\section{FUNDING}

Regione Toscana PAR FAS 2007-2013, Bando FAS Salute 2014, DAPHNE project.

\section{ACKNOWLEDGMENTS}

This work is financially supported by the DAPHNE project (Regione Toscana PAR FAS 2007-2013, Bando FAS Salute 2014, CUP J52I16000170002).

\section{SUPPLEMENTARY MATERIAL}

The Supplementary Material for this article can be found online at: https://www.frontiersin.org/articles/10.3389/fnins. 2017.00555/full\#supplementary-material

Berg, D., Lang, A. E., Postuma, R. B., Maetzler, W., Deuschl, G., Gasser, T., et al. (2013). Changing the research criteria for the diagnosis of Parkinson's disease: obstacles and opportunities. Lancet Neurol. 12, 514-524. doi: 10.1016/S1474-4422(13)70047-4

Bonato, P. (2010). Wearable sensors and systems. IEEE Eng. Med. Biol. Mag. 29, 25-36. doi: 10.1109/MEMB.2010.936554

Braybrook, M., O’Connor, S., Churchward, P., Perera, T., Farzanehfar, P., and Horne, M. (2016). An ambulatory tremor score for Parkinson's disease. J. Parkinsons. Dis. 6, 723-731. doi: 10.3233/JPD-160898

Brodie, M. A., Lovell, N. H., Canning, C. G., Menz, H. B., Delbaere, K., Redmond, S. J., et al. (2014). "Gait as a biomarker? Accelerometers reveal that reduced movement quality while walking is associated with Parkinson's disease, ageing and fall risk," in 36th Annual International Conference of the IEEE Engineering in Medicine and Biology Society (EMBC) (Chicago, IL: IEEE), 5968-5971.

Butt, A. H., Rovini, E., Esposito, D., Rossi, G., Maremmani, C., and Cavallo, F. (2017). Biomechanical parameter assessment for classification of Parkinson's disease on clinical scale. Int. J. Distrib. Sens Netw. 13, 1-15. doi: 10.1177/1550147717707417

Cabestany, J., Perez Lopez, C., Sama, A., Moreno, J. M., Bayes, A., and Rodriguez-Molinero, A. (2013). "REMPARK: When AI and technology meet Parkinson Disease assessment," in 20th International Conference Mixed Design of Integrated Circuits and Systems (MIXDES) (Gdynia: IEEE), 562-567.

Caldara, M., Comotti, D., Galizzi, M., Locatelli, P., Re, V., Alimonti, D., et al. (2014). "A novel body sensor network for Parkinson's disease patients rehabilitation assessment," in 11th International Conference on Wearable and Implantable Body Sensor Networks (BSN) (Zurich: IEEE), 81-68.

Cancela, J., Pansera, M., Arredondo, M. T., Estrada, J. J., Pastorino, M., PastorSanz, L., et al. (2010). "A comprehensive motor symptom monitoring and management system: The bradykinesia case," in 32nd Annual International Conference of the IEEE Engineering in Medicine and Biology Society (EMBC) (Buenos Aires: IEEE), 1008-1011.

Cancela, J., Pastorino, M., Arredondo, M. T., Pansera, M., Pastor-Sanz, L., Villagra, F., et al. (2011). "Gait assessment in Parkinson's disease patients through a network of wearable accelerometers in unsupervised environments," in 33rd Annual International Conference of the IEEE Engineering in Medicine and Biology Society (EMBC) (Boston, MA), 2233-2236.

Capecci, M., Pepa, L., Verdini, F., and Ceravolo, M. G. (2016). A smartphone-based architecture to detect and quantify freezing of gait in Parkinson's disease. Gait Posture 50, 28-33. doi: 10.1016/j.gaitpost.2016.08.018

Carter, J. H., Lyons, K. S., Lindauer, A., and Malcom, J. (2012). Pre-death grief in Parkinson's caregivers: a pilot survey-based study. Park. Relat. Disord. 18, S15-S18. doi: 10.1016/j.parkreldis.2012.06.015

Caslake, R., Taylor, K., Scott, N., Gordon, J., Harris, C., Wilde, K., et al. (2013). Age-, gender-, and socioeconomic status-specific incidence of Parkinson's disease and parkinsonism in North East Scotland: the PINE study. Park. Relat. Disord. 19, 515-521. doi: 10.1016/j.parkreldis.2013.01.014 
Caudron, S., Guerraz, M., Eusebio, A., Gros, J. P., Azulay, J. P., and Vaugoyeau, M. (2014). Evaluation of a visual biofeedback on the postural control in Parkinson's disease. Neurophysiol. Clin. 44, 77-86. doi: 10.1016/j.neucli.2013.10.134

Cavallo, F., Esposito, D., Rovini, E., Aquilano, M., Carrozza, M. C., Dario, P., et al. (2013). "Preliminary evaluation of SensHand V1 in assessing motor skills performance in Parkinson disease," in 13th International Conference on Rehabilitation Robotics (ICORR) (Seattle, WA: IEEE), 1-6.

Chen, T.-Z., Xu, G.-J., Zhou, G.-A., Wang, J.-R., Chan, P., and Du, Y.-F. (2014). Postural sway in idiopathic rapid eye movement sleep behavior disorder: a potential marker of prodromal Parkinson's disease. Brain Res. 1559, 26-32. doi: 10.1016/j.brainres.2014.02.040

Chou, K. L. (2008). Adverse events from the treatment of Parkinson's disease. Neurol. Clin. 26, S65-S83. doi: 10.1016/j.ncl.2008.05.003

Cole, B. T., Roy, S. H., and Nawab, S. H. (2011). "Detecting Freezing-of-Gait during unscripted and unconstrained activity," in 33rd Annual International Conference of the IEEE Engineering in Medicine and Biology Society (EMBC) (Boston, MA: IEEE), 5649-5652.

Cook, D. J., Schmitter-Edgecombe, M., and Dawadi, P. (2015). Analyzing activity behavior and movement in a naturalistic environment using smart home. J. Biomed. Heal Informatics 19, 1882-1892. doi: 10.1109/JBHI.2015.2461659

Curtze, C., Nutt, J. G., Carlson-Kuhta, P., Mancini, M., and Horak, F. B. (2016). Objective gait and balance impairments relate to balance confidence and perceived mobility in people with Parkinson's disease. Phys. Ther. 96, 1734-1743. doi: 10.2522/ptj.20150662

Daneault, J.-F., Carignan, B., Codère, C. É., Sadikot, A. F., and Duval, C. (2012). Using a smart phone as a standalone platform for detection and monitoring of pathological tremors. Front. Hum. Neurosci. 6:357. doi: 10.3389/fnhum.2012.00357

de Lau, L. M. L., Verbaan, D., Marinus, J., and Van Hilten, J. J. (2014). Survival in Parkinson's disease. Relation with motor and non-motor features. Park. Relat. Disord. 20, 613-616. doi: 10.1016/j.parkreldis.2014.02.030

Del Din, S., Godfrey, A., and Rochester, L. (2016). Validation of an accelerometer to quantify a comprehensive battery of gait characteristics in healthy older adults and Parkinson's disease: toward clinical and at home use. IEEE J. Biomed. Health Inform. 20, 838-847. doi: 10.1109/JBHI.2015.2419317

Delrobaei, M., Tran, S., Gilmore, G., McIsaac, K., and Jog, M. (2016). Characterization of multi-joint upper limb movements in a single task to assess bradykinesia. J. Neurol. Sci. 368, 337-342. doi: 10.1016/j.jns.2016.07.056

Delval, A., Tard, C., and Defebvre, L. (2014). Why we should study gait initiation in Parkinson's disease. Neurophysiol. Clin. Neurophysiol. 44, 69-76. doi: 10.1016/j.neucli.2013.10.127

Demonceau, M., Donneau, A. F., Croisier, J. L., Skawiniak, E., Boutaayamou, M., Maquet, D., et al. (2015). Contribution of a trunk accelerometer system to the characterization of gait in patients with mild-to-moderate Parkinson's disease. IEEE J. Biomed. Heal Informatics 19, 1803-1808. doi: 10.1109/JBHI.2015.2469540

Dickson, J. M., and Grünewald, R. A. (2004). Somatic symptom progression in idiopathic Parkinson's disease. Park. Relat. Disord. 10, 487-492. doi: 10.1016/j.parkreldis.2004.05.005

Djurić-Jovičić, M., Bobić, V. N., Ječmenica-Lukić, M., Petrović, I., Radovanović, S., Jovicic, N. S., et al. (2014a). "Implementation of a continuous wavelet transformation in repetitive finger tapping analysis for patients with PD," in 22nd Telecommunications Forum (TELFOR) (Belgrade), 541-544.

Djurić-Jovičić, M., Jovičić, N. S., Radovanović, S. M., Stanković, I. D., Popović, M. B., and Kostić, V. S. (2014b). Automatic identification and classification of Freezing of Gait episodes in Parkinson's disease patients. IEEE Trans. Neural Syst. Rehabil. Eng. 22, 685-694. doi: 10.1109/TNSRE.2013.2287241

Djurić-Jovičić, M., Petrović, I., Ječmenica-Lukić, M., Radovanović, S., DragaševićMišković, N., Belić, M., et al. (2016). Finger tapping analysis in patients with Parkinson's disease and atypical parkinsonism. J. Clin. Neurosci. 30, 49-55. doi: 10.1016/j.jocn.2015.10.053

Dockx, K., Bekkers, E. M., den Bergh, P., Van, G. P., Rochester, L., Hausdorff, J. M., et al. (2016). Virtual reality for rehabilitation in Parkinson's Disease. Cochrane Database Syst. Rev. 12:CD010760. doi: 10.1002/14651858.CD010760.pub2

Eskofier, B. M., Lee, S. I., Daneault, J. F., Golabchi, F. N., Ferreira-Carvalho, G., Vergara-Diaz, G., et al. (2016). "Recent machine learning advancements in sensor-based mobility analysis: deep learning for Parkinson's disease assessment," in 38th Annual International Conference of the IEEE Engineering in Medicine and Biology Society (EMBC) (Orlando, FL: IEEE), 655-658.

Esser, P., Dawes, H., Collett, J., and Howells, K. (2013). Insights into gait disorders: walking variability using phase plot analysis, Parkinson's disease. Gait Posture 38, 648-652. doi: 10.1016/j.gaitpost.2013.02.016

Fahn, S. (2008). “Clinical aspects of Parkinson disease," in Parkinson's Disease: Molecular and Therapeutic Insights from Model Systems, 1st Edn., eds R. Nass, S. Przedborski (Amsterdam: Elsevier Inc.), 3-48.

Fahn, S., and Elton, R. L. (1987). "UPDRS Development Committee. Unified Parkinson's Disease Rating Scale," in Recent Developments in Parkinson's Disease, eds S. Fahn, C. D. Marsden, D. B. Calne, and M. Goldstein (London: Macmillan), 153-304.

Fatmehsari, Y. R., and Bahrami, F. (2010). “Assessment of Parkinson's disease: classification and complexity analysis," in 17th Iran Conference of Biomedical Engineering (ICBME) (Isfahan: IEEE), 1-4.

Ferrari, A., Ginis, P., Hardegger, M., Casamassima, F., Rocchi, L., and Chiari, L. (2016). A mobile Kalman-filter based solution for the real-time estimation of spatio-temporal gait parameters. IEEE Trans. Neural Syst. Rehabil. Eng. 24, 764-773. doi: 10.1109/TNSRE.2015.2457511

Fisher, J. M., Hammerla, N. Y., Ploetz, T., Andras, P., Rochester, L., and Walker, R. W. (2016). Unsupervised home monitoring of Parkinson's disease motor symptoms using body-worn accelerometers. Park. Relat. Disord. 33, 44-50. doi: 10.1016/j.parkreldis.2016.09.009

Fukawa, K., Okuno, R., Yokoe, M., Sakoda, S., and Akazawa, K. (2007). "Estimation of UPDRS finger tapping score by using Artificial Neural Network for quantitative diagnosis of Parkinson's disease," in 6th International Special Topic Conference on ITAB (Tokyo: IEEE), 259-260.

Fukumoto, I. (2014). "A study for automatic diagnosing system of Parkinson disease: a systematic analysis of Parkinsonian tremor by accelerometer," in 11th IntConf.on Informatics in Control, Automation and Robotics (ICINCO) (Vienna: IEEE), 164-168.

Gago, M. F., Fernandez, V., Ferreira, J., Silva, H., Rodrigues, M. L., Rocha, L., et al. (2015). The effect of levodopa on postural stability evaluated by wearable inertial measurement units for idiopathic and vascular Parkinson's disease. Gait Posture 41, 459-464. doi: 10.1016/j.gaitpost.2014.11.008

Ghassemi, N. H., Marx Reiter, F., Pasluosta, C. F., Kugler, P., Schramm, A., Eskofier, B. M., et al. (2016). "Combined accelerometer and EMG analysis to differentiate," in 38th Annual International Conference of the IEEE Engineering in Medicine and Biology Society (EMBC) (Orlando, ON: IEEE), 672-675.

Ginis, P., Nieuwboer, A., Dorfman, M., Ferrari, A., Gazit, E., Canning, C. G., et al. (2016). Feasibility and effects of home-based smartphonedelivered automated feedback training for gait in people with Parkinson's disease: a pilot randomized controlled trial. Park. Relat. Disord. 22, 28-34. doi: 10.1016/j.parkreldis.2015.11.004

Giuberti, M., Ferrari, G., Contin, L., Cimolin, V., Azzaro, C., Albani, G., et al. (2014). "Linking UPDRS scores and kinematic variables in the leg agility task of Parkinsonians," in 11th International Conference on Wearable and Implantable Body Sensor Networks (BSN) (Zurich: IEEE), 115-120.

Giuberti, M., Ferrari, G., Contin, L., Cimolin, V., Azzaro, C., Albani, G., et al. (2015). Automatic UPDRS evaluation in the sit-to-stand task of parkinsonians: kinematic analysis and comparative outlook on the leg agility task. IEEE J. Biomed. Heal Informatics 19, 803-814. doi: 10.1109/JBHI.2015.2425296

Goetz, C. G., Tilley, B. C., Shaftman, S. R., Stebbins, G. T., Fahn, S., Martinez-Martin, P., et al. (2008). Movement Disorder Society-sponsored revision of the Unified Parkinson's Disease Rating Scale (MDS-UPDRS): scale presentation and clinimetric testing results. Mov. Disord. 23, 2129-2170. doi: $10.1002 / \mathrm{mds} .22340$

Grimpampi, E., Bonnet, V., Taviani, A., and Mazz, C. (2013). Estimate of lower trunk angles in pathological gaits using gyroscope data. Gait Posture 38, 523-527. doi: 10.1016/j.gaitpost.2013.01.031

Handojoseno, A. M. A., Shine, J. M., Gilat, M., Nguyen, T. N., Tran, Y., Lewis, S. J. G., et al. (2014). "Prediction of freezing of gait using analysis of brain effective connectivity," in 36th Annual International Conference of the IEEE Engineering in Medicine and Biology Society (EMBC) (Chicago, IL: IEEE), 4119-4122.

Handojoseno, A. M. A., Shine, J. M., Nguyen, T. N., Tran, Y., Lewis, S. J. G., and Nguyen, H. T. (2012). "The detection of Freezing of Gait in Parkinson's disease patients using EEG signals based on Wavelet decomposition," in 34th 
Annual International Conference of the IEEE Engineering in Medicine and Biology Society (EMBC) (San Diego, CA: IEEE), 69-72.

Handojoseno, A. M. A., Shine, J. M., Nguyen, T. N., Tran, Y., Lewis, S. J. G., and Nguyen, H. T. (2013). "Using EEG spatial correlation, cross frequency energy, and wavelet coefficients for the prediction of Freezing of Gait in Parkinson's Disease patients," in 35th Annual International Conference of the IEEE Engineering in Medicine and Biology Society (EMBC) (Osaka: IEEE), $4263-4266$.

Handojoseno, A. M. A., Shine, J. M., Nguyen, T. N., Tran, Y., Lewis, S. J. G., and Nguyen, H. T. (2015). Analysis and prediction of the Freezing of Gait using EEG brain dynamics. IEEE Trans. Neural Syst. Rehabil. Eng. 23, 887-896. doi: 10.1109/TNSRE.2014.2381254

Henderson, E. J., Lord, S. R., Brodie, M. A., Gaunt, D. M., Lawrence, A. D., Close, J. C. T., et al. (2016). Rivastigmine for gait stability in patients with Parkinson's disease (ReSPonD): a randomised, double-blind, placebo-controlled, phase 2 trial. Lancet Neurol. 15, 249-258. doi: 10.1016/S1474-4422(15)00389-0

Hill, E., Stuart, S., Lord, S., Del Din, S., and Rochester, L. (2016). Vision, visuocognition and postural control in Parkinson's disease: an associative pilot study. Gait Posture. 48, 74-76. doi: 10.1016/j.gaitpost.2016.04.024

Hoehn, M. M., and Yahr, M. D. (1967). Parkinsonism: onset, progression, and mortality. Neurology 17, 427-442. doi: 10.1212/WNL.17.5.427

Hoffman, J. D., and McNames, J. (2011). "Objective measure of upper extremity motor impairment in Parkinson's disease with inertial sensors," in 33rd Annual International Conference of the IEEE Engineering in Medicine and Biology Society (EMBC) (Boston, MA: IEEE), 4378-4381.

Horak, F. B., Mancini, M., Carlson-Kuhta, P., Nutt, J. G., and Salarian, A. (2016). Balance and Gait represent independent domains of mobility in Parkinson Disease. Phys. Ther. 96, 1364-1371. doi: 10.2522/ptj.20150580

Hossen, A. (2012). "Selection of wavelet-bands for neural network discrimination of Parkinsonian tremor from essential tremor," in 19th International Conference on Electronics, Circuits, and Systems (ICECS) (Sevilla: IEEE), 37-40.

Hossen, A., Muthuraman, M., Al-Hakim, Z., Raethjen, J., Deuschl, G., and Heute, U. (2013). Discrimination of Parkinsonian tremor from essential tremor using statistical signal characterization of the spectrum of accelerometer signal. Biomed. Mater. Eng. 23, 513-531. doi: 10.3233/BME-130773

Hossen, A., Muthuraman, M., Raethjen, J., Deuschl, G., and Heute, U. (2010). Discrimination of parkinsonian tremor from essential tremor by implementation of a wavelet-based soft-decision technique on emg and accelerometer signals. Biomed. Signal Process. Control. 5, 181-188. doi: 10.1016/j.bspc.2010.02.005

Hssayeni, M. D., Burack, M. A., and Ghoraani, B. (2016). "Automatic assessment of medication states of patients with Parkinson's disease using wearable sensors," in 38th Annual International Conference of the IEEE Engineering in Medicine and Biology Society (EMBC) (Orlando, FL: IEEE), 6082-6085.

Hu, M. T. M., Butterworth, R., Kumar, V., Cooper, J., Jones, E., Catterall, L., et al. (2011). How common and what are the determinants of sub-optimal care for Parkinson's disease patients: the Milton Keynes community study. Park. Relat. Disord. 17, 177-181. doi: 10.1016/j.parkreldis.2010.12.012

Hubble, R. P., Silburn, P. A., Naughton, G. A., and Cole, M. H. (2016). Assessing stability in mild and moderate Parkinson's disease: can clinical measures provide insight? Gait Posture 49, 7-13. doi: 10.1016/j.gaitpost.2016.06.002

Hwang, I. S., Lin, C. C. K., and Wu, P. S. (2009). Tremor modulation in patients with Parkinson's disease compared to healthy counterparts during loaded postural holding. J. Electromyogr. Kinesiol. 19, e520-e528. doi: 10.1016/j.jelekin.2009.03.005

Jarchi, D., Peters, A., Lo, B., Kalliolia, E., Giulio, I., Di Limousin, P., et al. (2015). "Assessment of the e-AR sensor for gait analysis of Parkinson's disease patients," in 12th International Conference on Wearable and Implantable Body Sensor Networks (BSN) (Cambridge, MA: IEEE), 1-6.

Jaywant, A., Ellis, T. D., Roy, S., Lin, C. C., Neargarder, S., and Cronin-Golomb, A. (2016). Randomized controlled trial of a home-based action observation intervention to improve walking in Parkinson disease. Arch. Phys. Med. Rehabil. 97, 665-673. doi: 10.1016/j.apmr.2015.12.029

Jia, X., Duroseau, N., Chan, V., Ciraco, C., Wang, R., Nia, S. M., et al. (2014). "Objective quantification of upper extremity motor functions in Unified Parkinson's Disease Rating Scale Test," in 36th Annual International Conference of the IEEE Engineering in Medicine and Biology Society (EMBC) (Chicago, IL: IEEE), 5345-5348.
Kassubek, J. (2014). Diagnostic procedures during the course of Parkinson's Disease. Basal Ganglia. 4, 15-18. doi: 10.1016/j.baga.2014.02.001

Khan, F. M., Barnathan, M., Montgomery, M., Myers, S., Cot,é, L., and Loftus, S. (2014). "A wearable accelerometer sensor system for unobtrusive real time monitoring of Parkinson disease motor symptoms," in International Conference on Bioinformatics and Bioengineering (Boca Raton, FL), 120-125.

Korczyn, A. D., and Gurevich, T. (2010). Parkinson's disease: before the motor symptoms and beyond. J. Neurol. Sci. 289, 2-6. doi: 10.1016/j.jns.2009.08.032

Kostikis, N., Arnaoutoglou, M., and Kotsavasiloglou, C. (2015). A Smartphonebased tool for assessing Parkinsonian hand tremor. J. Biomed. Heal Informat. 19, 1835-1842. doi: 10.1109/JBHI.2015.2471093

Kwon, K. Y., Lee, H. M., Lee, S. M., Kang, S. H., and Koh, S. B. (2016). Comparison of motor and non-motor features between essential tremor and tremor dominant Parkinson's disease. J. Neurol. Sci. 361, 34-38. doi: 10.1016/j.jns.2015.12.016

Lambrecht, S., Romero, J. P., Benito-León, J., Rocon, E., and Pons, J. L. (2014). "Task independent identification of sensor location on upper limb from orientation data," in 36th Annual International Conference of the IEEE Engineering in Medicine and Biology Society (EMBC) (Chicago, IL: IEEE), 6627-6630.

Lord, S., Rochester, L., Hetherington, V., Allcock, L. M., and Burn, D. (2010). Executive dysfunction and attention contribute to gait interference in "off" state Parkinson's Disease. Gait Posture. 31, 169-174. doi: 10.1016/j.gaitpost.2009.09.019

Lorenzi, P., Rao, R., Romano, G., Kita, A., Serpa, M., Filesi, F., et al. (2015). Smart sensing systems for the detection of human motion disorders. Proc. Eng. 120, 324-327. doi: 10.1016/j.proeng.2015.08.626

Mailankody, P., Thennarasu, K., Nagaraju, B. C., Yadav, R., and Pal, P. K. (2016). Re-emergent tremor in Parkinson's disease: a clinical and electromyographic study. J. Neurol. Sci. 366, 33-36. doi: 10.1016/j.jns.2016.04.041

Mancini, M., Chiari, L., Holmstrom, L., Salarian, A., and Horak, F. B. (2016). Validity and reliability of an IMU-based method to detect APAs prior to gait initiation. Gait Posture 43, 125-131. doi: 10.1016/j.gaitpost.2015.08.015

Mancini, M., Horak, F. B., Zampieri, C., Carlson-Kuhta, P., Nutt, J. G., and Chiari, L. (2011). Trunk accelerometry reveals postural instability in untreated Parkinson's disease. Park Relat Disord. 17, 557-562. doi: 10.1016/j.parkreldis.2011.05.010

Mariani, B., Jiménez, M. C., Vingerhoets, F. J. G., and Aminian, K. (2013). On-shoe wearable sensors for gait and turning assessment of patients with Parkinson's disease. IEEE Trans. Biomed. Eng. 60, 155-158. doi: 10.1109/TBME.2012.2227317

Masu, K., Yamane, D., Machida, K., Sone, M., and Miyake, Y. (2016). "Development of high sensitivity CMOS-MEMS inertia sensor and its application to early-stage diagnosis of Parkinson's disease," in 46th European Solid-State Device Research Conference (Lausanne), 91-96.

Mazilu, S., Blanke, U., Calatroni, A., Gazit, E., Hausdorff, J. M., and Tröster, G. (2016). The role of wrist-mounted inertial sensors in detecting gait freeze episodes in Parkinson's disease. Pervasive Mob. Comput. 33, 1-16. doi: 10.1016/j.pmcj.2015.12.007

Mazilu, S., Blanke, U., Hardegger, M., Tr, G., Gazit, E., Dorfman, M., et al. (2014). "GaitAssist: a wearable assistant for gait training and rehabilitation in Parkinson's disease," in International Conference on Pervasive Computing and Communication (PerCom) (Budapest: IEEE), 1-3.

Mazilu, S., Calatroni, A., Gazit, E., Mirelman, A., Hausdorff, J. M., and Tröster, G. (2015). Prediction of Freezing of Gait in Parkinson's from physiological wearables: an exploratory study. J. Biomed. Heal Informat. 19, 1843-1854. doi: $10.1109 /$ JBHI.2015.2465134

Mazilu, S., and Hardegger, M. (2012). "Online detection of freezing of gait with smartphones and machine learning techniques," in 6th International Conference on Pervasive Computing Technologies for Healthcare (PervasiveHealth) (San Diego, CA), 123-130.

Megalingam, R. K., Pocklassery, G., Jayakrishnan, V., Mourya, G., and Thulasi, A. A. (2014). "Smartphone based continuous monitoring system for home-bound elders and patients," in International Conference on Communication and Signal Processing (ICCSP) (Chennai: IEEE), 1173-1177.

Mellone, S., Palmerini, L., Cappello, A., and Chiari, L. (2011). Hilbert-huang-based tremor removal to assess postural properties from accelerometers. IEEE Trans. Biomed. Eng. 58, 1752-1761. doi: 10.1109/TBME.2011.2116017 
Mirelman, A., Gurevich, T., Giladi, N., Bar-Shira, A., Orr-Urtreger, A., and Hausdorff, J. M. (2011). Gait alterations in healthy carriers of the LRRK2 G2019S mutation. Ann. Neurol. 69, 193-197. doi: 10.1002/ana.22165

Moore, S. T., MacDougall, H. G., and Ondo, W. G. (2008). Ambulatory monitoring of freezing of gait in Parkinson's disease. J. Neurosci. Methods 167, 340-348. doi: 10.1016/j.jneumeth.2007.08.023

Morris, T. R., Cho, C., Dilda, V., Shine, J. M., Naismith, S. L., Lewis, S. J. G., et al. (2012). A comparison of clinical and objective measures of freezing of gait in Parkinson's disease. Park Relat. Disord. 18, 572-577. doi: 10.1016/j.parkreldis.2012.03.001

Morris, T. R., Cho, C., Dilda, V., Shine, J. M., Naismith, S. L., Lewis, S. J. G., et al. (2013). Clinical assessment of freezing of gait in Parkinson's disease from computer-generated animation. Gait Posture 38, 326-329. doi: 10.1016/j.gaitpost.2012.12.011

Niazmand, K., Tonn, K., Kalaras, A., Kammermeier, S., Boetzel, K., Mehrkens, J. H., et al. (2011). "A measurement device for motion analysis of patients with Parkinson's disease using sensor based smart clothes," in Pervasive Comput Technol Healthc (PervasiveHealth), 2011 5th International Conference (Dublin), 9-16.

Okuno, R., Yokoe, M., Fukawa, K., Sakoda, S., and Akazawa, K. (2007). "Measurement system of finger-tapping contact force for quantitative diagnosis of Parkinson's disease," in 29th Annual International Conference of the IEEE Engineering in Medicine and Biology Society (EMBC) (Lyon: IEEE), 1354-1357.

Ossig, C., and Reichmann, H. (2015). Treatment strategies in early and advanced Parkinson disease. Neurol. Clin. 33, 19-37. doi: 10.1016/j.ncl.2014.09.009

Oung, Q. W., Hariharan, M., Lee, H. L., Basah, S. N., Sarillee, M., and Lee, C. H. (2015). "Wearable multimodal sensors for evaluation of patients with Parkinson disease," in 5th International Conference on Control System, Computing and Engineering (ICCSCE) (Penang: IEEE), 269-274.

Ozinga, S. J., Linder, S. M., and Alberts, J. L. (2017). Use of mobile device accelerometry to enhance evaluation of postural instability in Parkinson's disease. Arch. Phys. Med. Rehabil. 98, 649-658. doi: 10.1016/j.apmr.2016. 08.479

Palma, J.-A., and Kaufmann, H. (2014). Autonomic disorders predicting Parkinson disease. Park Relat. Disord. 20, S94-S98. doi: 10.1016/S1353-8020(13)70024-5

Palmerini, L., Mellone, S., Avanzolini, G., Valzania, F., and Chiari, L. (2013). Quantification of motor impairment in Parkinson's disease using an instrumented timed up and go test. IEEE Trans. Neural Syst. Rehabil. Eng. 21, 664-673. doi: 10.1109/TNSRE.2012.2236577

Palmerini, L., Rocchi, L., Mellone, S., Valzania, F., and Chiari, L. (2011). Feature selection for accelerometer-based posture analysis in Parkinson's disease. IEEE Trans. Inf. Technol. Biomed. 15, 481-490. doi: 10.1109/TITB.2011.2107916

Paredes, A. J. D., Munoz, B., Agredo, W., Ariza-Araujo, Y., Luis Orozco, J., and Navarro, A. (2015). "A reliability assessment software using Kinect to complement the clinical evaluation of Parkinson's disease," in 37th Annual International Conference of the IEEE Engineering in Medicine and Biology Society (EMBC) (Milan: IEEE), 6860-6863.

Parisi, F., Ferrari, G., Giuberti, M., Contin, L., Cimolin, V., Azzaro, C., et al. (2015). Body-sensor-network-based kinematic characterization and comparative outlook of UPDRS scoring in leg agility, sit-to-stand, and Gait tasks in Parkinson's disease. J. Biomed. Heal Informat. 19, 1777-1793. doi: 10.1109/JBHI.2015.2472640

Parisi, F., Ferrari, G., Giuberti, M., Contin, L., Cimolin, V., Azzaro, C., et al. (2016). Inertial BSN-based characterization and automatic UPDRS evaluation of the gait task of Parkinsonians. IEEE Trans. Affect Comput. 7, 258-271. doi: 10.1109/TAFFC.2016.2549533

Pasluosta, C. F., Barth, J., Gassner, H., Klucken, J., and Eskofier, B. M. (2015). "Pull test estimation in Parkinson's disease patients using wearable sensor technology," in 37th Annual International Conference of the IEEE Engineering in Medicine and Biology Society (EMBC) (Milan: IEEE), 3109-3112.

Pastorino, M., Cancela, J., Arredondo, M. T., Pansera, M., Pastor-Sanz, L., Villagra, F., et al. (2011). "Assessment of bradykinesia in Parkinson's disease patients through a multi-parametric system," in 33rd Annual International Conference of the IEEE Engineering in Medicine and Biology Society (EMBC) (Boston, MA: IEEE), 1810-1813.

Patel, S., Lorincz, K., Hughes, R., Huggins, N., Growdon, J., Standaert, D., et al. (2009). Monitoring motor fluctuations in patients with Parkinson's disease using wearable sensors. IEEE Trans. Inf. Technol. Biomed. 13, 864-873. doi: 10.1109/TITB.2009.2033471

Pérez-López, C., Samà, A., Rodríguez-Martín, D., Moreno-Aróstegui, J. M., Cabestany, J., Bayes, A., et al. (2016). Dopaminergic-induced dyskinesia assessment based on a single belt-worn accelerometer. Artif. Intell. Med. 67, 47-56. doi: 10.1016/j.artmed.2016.01.001

Perumal, S. V., and Sankar, R. (2016). Gait and tremor assessment for patients with Parkinson's disease using wearable sensors. ICT Expr. 2, 168-174. doi: 10.1016/j.icte.2016.10.005

Pierleoni, P., Palma, L., Belli, A., and Pernini, L. (2014). “A real-time system to aid clinical classification and quantification of tremor in Parkinson's disease," in International Conference on Biomedical and Health Informatics (BHI) (Valencia: IEEE), 113-116.

Rahimi, F., Bee, C., Duval, C., Boissy, P., Edwards, R., and Jog, M. (2014). Using ecological whole body kinematics to evaluate effects of medication adjustment in Parkinson disease. J. Parkinsons. Dis. 4, 617-627. doi: 10.1109/IEMBS.2011.6091443

Rahimi, F., Duval, C., Jog, M., Bee, C., Ma, A. S., Jog, M., et al. (2011). “Capturing whole-body mobility of patients with Parkinson disease using inertial motion sensors : Expected challenges and rewards," in 33rd Annual International Conference of the IEEE Engineering in Medicine and Biology Society (EMBC) (Boston, MA: IEEE), 5833-5838.

Ramsperger, R., Meckler, S., Heger, T., van Uem, J., Hucker, S., Braatz U., et al. (2016). Continuous leg dyskinesia assessment in Parkinson's disease -clinical validity and ecological effect. Park Relat. Disord. 26, 41-46. doi: 10.1016/j.parkreldis.2016.02.007

Razali, R., Ahmad, F., Rahman, F. N. A., Midin, M., and Sidi, H. (2011). Burden of care among caregivers of patients with Parkinson disease: a cross-sectional study. Clin. Neurol Neurosurg. 113, 639-643. doi: 10.1016/j.clineuro.2011.05.008

Reinfelder, S., Hauer, R., Barth, J., Klucken, J., and Eskofier, B. M. (2015). “Timed Up-and-Go phase segmentation in Parkinson's disease patients using unobtrusive inertial sensors," in 37th Annual International Conference of the IEEE Engineering in Medicine and Biology Society (EMBC) (Milan: IEEE), 5171-5174.

Rigas, G., Gatsios, D., Fotiadis, D. I., Chondrogiorgi, M., Tsironis, C., Konitsiotis, S., et al. (2016). "Tremor UPDRS estimation in home environment," in 38th Annual International Conference of the IEEE Engineering in Medicine and Biology Society (EMBC) (Orlando, FL: IEEE), 3642-3645.

Rigas, G., Tzallas, A. T., Tsipouras, M. G., Bougia, P., Tripoliti, E. E., Baga, D., et al. (2012). Assessment of tremor activity in the Parkinson's Disease using a set of wearable sensors. IEEE Trans. Inf. Technol. Biomed. 16, 478-487. doi: 10.1109/TITB.2011.2182616

Rissanen, S. M., Kankaanpaa, M., Tarvainen, M. P., Meigal, A. Y., Nuutinen, J., Tarkka, I. M., et al. (2009). Analysis of dynamic voluntary muscle contractions in Parkinson's disease. IEEE Trans. Biomed. Eng. 56, 2280-2288. doi: 10.1109/TBME.2009.2023795

Rissanen, S. M., Kankaanp,ää, M., Tarvainen, M. P., Novak, V., Novak, P., Hu, K., et al. (2011). Analysis of EMG and acceleration signals for quantifying the effects of deep brain stimulation in Parkinson's disease. IEEE Trans. Biomed. Eng. 58, 2545-2553. doi: 10.1109/TBME.2011.2159380

Rissanen, S. M., Ruonala, V., Pekkonen, E., Kankaanp,ää, M., Airaksinen, O., and Karjalainen, P. A. (2015). Signal features of surface electromyography in advanced Parkinson's disease during different settings of deep brain stimulation. Clin. Neurophysiol. 126, 2290-2298. doi: 10.1016/j.clinph.2015.01.021

Robles-García, V., Arias, P., Sanmartín, G., Espinosa, N., Flores, J., Grieve, K. L., et al. (2013). Motor facilitation during real-time movement imitation in Parkinson's disease: a virtual reality study. Park Relat. Disord. 19, 1123-1129. doi: 10.1016/j.parkreldis.2013.08.005

Rocchi, L., Palmerini, L., Weiss, A., Herman, T., and Hausdorff, J. M. (2014). Balance testing with inertial sensors in patients with Parkinson's disease: assessment of motor subtypes. IEEE Trans. Neural Syst. Rehabil. Eng. 22, 1064-1071. doi: 10.1109/TNSRE.2013.2292496

Rochester, L., Baker, K., Hetherington, V., Jones, D., Willems, A. M., Kwakkel, G., et al. (2010). Evidence for motor learning in Parkinson's disease: acquisition, automaticity and retention of cued gait performance after training with external rhythmical cues. Brain Res. 1319, 103-111. doi: 10.1016/j.brainres.2010.01.001 
Rodgers, M. M., Pai, V. M., and Conroy, R. S. (2015). Recent advances in wearable sensors for health monitoring. IEEE Sens. J. 15, 3119-3126. doi: 10.1109/JSEN.2014.2357257

Roland, K. P., Jones, G. R., and Jakobi, J. M. (2013). Parkinson's disease and sex-related differences in electromyography during daily life. J. Electromyogr. Kinesiol. 23, 958-965. doi: 10.1016/j.jelekin.2013.03.008

Roland, K. P., Jones, G. R., and Jakobi, J. M. (2014). Daily electromyography in females with Parkinson's disease: a potential indicator of frailty. Arch. Gerontol. Geriatr. 58, 80-87. doi: 10.1016/j.archger.2013.08.008

Rovini, E., Esposito, D., Maremmani, C., Bongioanni, P., and Cavallo, F. (2016). "Empowering patients in self-management of Parkinson's disease through cooperative ICT systems," in Optimizing Assistive Technologies for Aging Populations, eds Y. S. Morsi, A. Shukla, and C. P. Rathore (Hershey, PA: IGI Global), 251-277.

Roy, S. H., Cole, B. T., Member, S. S. S. S., Gilmore, L. D., Luca, C. J., and De Nawab, S. H., et al. (2011). "Resolving signal complexities for ambulatory monitoring of notor function in Parkinson's Disease," in 33rd Annual International Conference of the IEEE Engineering in Medicine and Biology Society (EMBC) (Boston, MA: IEEE), 4832-4835.

Ruonala, V., Meigal, A., Rissanen, S. M., Airaksinen, O., Kankaanpää, M., and Karjalainen, P. A. (2014a). EMG signal morphology and kinematic parameters in essential tremor and Parkinson's disease patients. J. Electromyogr. Kinesiol. 24, 300-306. doi: 10.1016/j.jelekin.2013.12.007

Ruonala, V., Pekkonen, E., Rissanen, S., Airaksinen, O., Miroshnichenko, G., Kankaanpää, M., et al. (2014b). "Dynamic tension EMG to characterize the effects of DBS treatment of advanced Parkinson's disease," in 36th Annual International Conference of the IEEE Engineering in Medicine and Biology Society (EMBC) (Chicago, IL: IEEE), 3248-3251.

Ruonala, V., Tarvainen, M. P., Karjalainen, P. A., Pekkonen, E., and Rissanen, S. M. (2015). "Autonomic nervous system response to L-dopa in patients with advanced Parkinson's disease," in 37th Annual International Conference of the IEEE Engineering in Medicine and Biology Society (EMBC) (Milan: IEEE), 6162-6165.

Salarian, A., Burkhard, P. R., Vingerhoets, F. J. G., Jolles, B. M., and Aminian, K. (2013). A novel approach to reducing number of sensing units for wearable gait analysis systems. IEEE Trans. Biomed. Eng. 60, 72-77. doi: 10.1109/TBME.2012.2223465

Salarian, A., Horak, F. B., Zampieri, C., Carlson-Kuhta, P., Nutt, J. G., and Aminian, K. (2010). ITUG, a sensitive and reliable measure of mobility. IEEE Trans. Neural Syst. Rehabil. Eng. 18, 303-310. doi: 10.1109/TNSRE.2010.2047606

Salarian, A., Russmann, H., Vingerhoets, F. J. G., Burkhard, P. R., and Aminian, K. (2007a). Ambulatory monitoring of physical activities in patients with Parkinson's disease. IEEE Trans. Biomed. Eng. 54, 2296-2299. doi: 10.1109/TBME.2007.896591

Salarian, A., Russmann, H., Wider, C., Burkhard, P. R., Vingerhoets, F. J. G., and Aminian, K. (2007b). Quantification of tremor and bradykinesia in Parkinson's disease using a novel ambulatory monitoring system. IEEE Trans Biomed Eng. 54, 313-322. doi: 10.1109/TBME.2006.886670

Salarian, A., Zampieri, C., Horak, F. B., Carlson-Kuhta, P., Nutt, J. G., and Aminian, K. (2009). "Analyzing $180^{\circ}$ turns using an inertial system reveals early signs of progression of Parkinson's disease," in 31st Annual International Conference of the IEEE Engineering in Medicine and Biology Society (EMBC) (Minneapolis, MN: IEEE), 224-227.

Samà, A., Perez Lopez, C., Romagosa, J., Rodriguez-Martin, D., Català, A., Cabestany, J., et al. (2012). “Dyskinesia and motor state detection in Parkinson's disease patients with a single movement sensor," in 34th Annual International Conference of the IEEE Engineering in Medicine and Biology Society (EMBC) (San Diego, CA: IEEE), 1194-1197.

Sant'Anna, A., Salarian, A., and Wickstr, N. (2011). A new measure of movement symmetry in early Parkinson's disease patients using symbolic processing of inertial sensor data. IEEE Trans. Biomed. Eng. 58, 2127-2135. doi: 10.1109/TBME.2011.2149521

Scanlon, B. K., Levin, B. E., Nation, D. A., Katzen, H. L., Guevara-Salcedo, A., Singer, C., et al. (2013). An accelerometry-based study of lower and upper limb tremor in Parkinson's disease. J. Clin. Neurosci. 20, 827-830. doi: 10.1016/j.jocn.2012.06.015
Schmidt, A. L., Pennypacker, M. L., Thrush, A. H., Leiper, C. I., and Craik, R. L. (2011). Validity of the StepWatch step activity monitor: preliminary findings for use in persons with Parkinson disease and multiple sclerosis. J. Geriatr. Phys. Ther. 34, 41-45. doi: 10.1519/JPT.0b013e31820aa921

Sejdić, E., Lowry, K. A., Bellanca, J., Perera, S., Redfern, M. S., and Brach, J. S. (2016). Extraction of stride events from gait accelerometry during treadmill walking. IEEE J. Transl. Eng. Heal. Med. 4, 1-11. doi: 10.1109/JTEHM.2015.2504961

Stamatakis, J., Crémers, J., Maquet, D., Macq, B., and Garraux, G. (2011). "Gait feature extraction in Parkinson's disease using low-cost accelerometers," in 33rd Annual International Conference of the IEEE Engineering in Medicine and Biology Society (EMBC) (Boston, MA: IEEE), 7900-7903.

Strauss, I., Kalia, S. K., and Lozano, A. M. (2014). Where are we with surgical therapies for Parkinson's disease? Park. Relat. Disord. 20, S187-S191. doi: 10.1016/S1353-8020(13)70044-0

Surangsrirat, D., Thanawattano, C., Pongthornseri, R., Dumnin, S., Anan, C., and Bhidayasiri, R. (2016). "Support vector machine classification of Parkinson's disease and essential tremor subjects based on temporal fluctuation," in 38th Annual International Conference of the IEEE Engineering in Medicine and Biology Society (EMBC) (Orlando, FL: IEEE), 6389-6392.

Szewczyk-Krolikowski, K., Tomlinson, P., Nithi, K., Wade-Martins, R., Talbot, K., Ben-Shlomo, Y., et al. (2014). The influence of age and gender on motor and non-motor features of early Parkinson's disease: initial findings from the Oxford Parkinson Disease Center (OPDC) discovery cohort. Park Relat. Disord. 20, 99-105. doi: 10.1016/j.parkreldis.2013.09.025

Thanawattano, C., Anan, C., Pongthornseri, R., Dumnin, S., and Bhidayasiri, R. (2015). “Temporal fluctuation analysis of tremor signal in Parkinson's disease and Essential tremor subjects," in 37th Annual International Conference of the IEEE Engineering in Medicine and Biology Society (EMBC) (Milan: IEEE), 6054-6057.

Tien, I., Glaser, S. D., and Aminoff, M. J. (2010). "Characterization of gait abnormalities in Parkinson's disease using a wireless inertial sensor system," in 32nd Annual International Conference of the IEEE Engineering in Medicine and Biology Society (EMBC) (Buenos Aires: IEEE), 3353-3356.

Tolosa, E., and Pont-Sunyer, C. (2011). Progress in defining the premotor phase of Parkinson's disease. J. Neurol. Sci. 310, 4-8. doi: 10.1016/j.jns.2011.05.027

Tolosa, E., Wenning, G. K., and Poewe, W. (2006). The diagnosis of Parkinson's disease. Lancet Neurol. 5, 75-86. doi: 10.1016/S1474-4422(05)70285-4

Tripoliti, E. E., Tzallas, A. T., Tsipouras, M. G., Rigas, G., Bougia, P., Leontiou, M., et al. (2013). Automatic detection of freezing of gait events in patients with Parkinson's disease. Comput. Methods Progr. Biomed. 110, 12-26. doi: 10.1016/j.cmpb.2012.10.016

Trojaniello, D., Ravaschio, A., Hausdorff, J. M., and Cereatti, A. (2015). Comparative assessment of different methods for the estimation of gait temporal parameters using a single inertial sensor: application to elderly, poststroke, Parkinson's disease and Huntington's disease subjects. Gait Posture 42, 310-316. doi: 10.1016/j.gaitpost.2015.06.008

Tsipouras, M. G., Tzallas, A. T., Fotiadis, D. I., and Konitsiotis, S. (2011). "On automated assessment of Levodopa-induced dyskinesia in Parkinson's disease," in 33rd Annual International Conference of the IEEE Engineering in Medicine and Biology Society (EMBC) (Boston, MA: IEEE), 2679-2682.

Tsipouras, M. G., Tzallas, A. T., Karvounis, E. C., Tsalikakis, D. G., Cancela, J., Pastorino, M., et al. (2014). “A wearable system for long-term ubiquitous monitoring of common motor symptoms in patients with Parkinson's disease," in International Conference on Biomedical and Health Informatics (BHI) (Valencia: IEEE), 173-176.

Tsipouras, M. G., Tzallas, A. T., Rigas, G., Tsouli, S., Fotiadis, D. I., and Konitsiotis, S. (2012). An automated methodology for levodopa-induced dyskinesia: assessment based on gyroscope and accelerometer signals. Artif. Intell. Med. 55, 127-135. doi: 10.1016/j.artmed.2012.03.003

Wallén, M. B., Nero, H., Franzén, E., and Hagströmer, M. (2014). Comparison of two accelerometer filter settings in individuals with Parkinson's disease. Physiol. Meas. 35, 2287-2296. doi: 10.1088/0967-3334/35/11/2287

Weiss, A., Herman, T., Plotnik, M., Brozgol, M., Maidan, I., Giladi, N., et al. (2010). Can an accelerometer enhance the utility of the Timed Up \& Go Test when evaluating patients with Parkinson's disease? Med. Eng. Phys. 32, 119-125. doi: 10.1016/j.medengphy.2009.10.015 
Weiss, A., Sharifi, S., Plotnik, M., van Vugt, J. P. P., Giladi, N., and Hausdorff, J. M. (2011). Toward automated, at-home assessment of mobility among patients with Parkinson disease, using a body-worn accelerometer. Neurorehabil. Neural Repair. 25, 810-818. doi: 10.1177/1545968311424869

Wolters, E. C. (2008). Variability in the clinical expression of Parkinson's disease. J. Neurol. Sci. 266, 197-203. doi: 10.1016/j.jns.2007.08.016

Yelshyna, D., Gago, M. F., Bicho, E., Fernandes, V., Gago, N. F., Costa, L., et al. (2016). Compensatory postural adjustments in Parkinson's disease assessed via a virtual reality environment. Behav. Brain Res. 296, 384-392. doi: 10.1016/j.bbr.2015.08.017

Yokoe, M., Okuno, R., Hamasaki, T., Kurachi, Y., Akazawa, K., and Sakoda, S. (2009). Opening velocity, a novel parameter, for finger tapping test in patients with Parkinson's disease. Park Relat. Disord. 15, 440-444. doi: 10.1016/j.parkreldis.2008.11.003

Yoneyama, M., Kurihara, Y., Watanabe, K., and Mitoma, H. (2013). Accelerometry-based gait analysis and its application to Parkinson's Disease assessment. Part 2: a new measure for quantifying walking behavior. IEEE Trans. Neural Syst. Rehabil. Eng. 21, 999-1005. doi: 10.1109/TNSRE.2013.2268251

Yoneyama, M., Mitoma, H., Sanjo, N., Higuma, M., Terashi, H., and Yokota, T. (2016). Ambulatory gait behavior in patients with dementia: a comparison with Parkinson's disease. IEEE Trans. Neural Syst. Rehabil. Eng. 24, 817-826. doi: 10.1109/TNSRE.2015.2477856

Zach, H., Janssen, A. M., Snijders, A. H., Delval, A., Ferraye, M. U., Auff, E., et al. (2015). Identifying freezing of gait in Parkinson's disease during freezing provoking tasks using waist-mounted accelerometry. Park. Relat. Disord. 21, 1362-1366. doi: 10.1016/j.parkreldis.2015. 09.051

Zhou, Y., Jenkins, M. E., Naish, M. D., and Trejos, A. L. (2016). “The measurement and analysis of Parkinsonian hand tremor," in International Conference on Biomedical and Health Informatics (BHI) (Las Vegas, NV: IEEE) 414-417.

Conflict of Interest Statement: The authors declare that the research was conducted in the absence of any commercial or financial relationships that could be construed as a potential conflict of interest.

Copyright (c) 2017 Rovini, Maremmani and Cavallo. This is an open-access article distributed under the terms of the Creative Commons Attribution License (CC BY). The use, distribution or reproduction in other forums is permitted, provided the original author(s) or licensor are credited and that the original publication in this journal is cited, in accordance with accepted academic practice. No use, distribution or reproduction is permitted which does not comply with these terms. 